University of San Diego

Digital USD

2015-5

\title{
Hispanic Mothers' Narratives: Experiences of Parenting a Child with Cystic Fibrosis, Health Beliefs, and Health Care Experiences Related to Cystic Fibrosis
}

Linda Tirabassi-Mathis

University of San Diego

Follow this and additional works at: https://digital.sandiego.edu/dissertations

\section{Digital USD Citation}

Tirabassi-Mathis, Linda, "Hispanic Mothers' Narratives: Experiences of Parenting a Child with Cystic Fibrosis, Health Beliefs, and Health Care Experiences Related to Cystic Fibrosis" (2015). Dissertations. 4. https://digital.sandiego.edu/dissertations/4

This Dissertation: Open Access is brought to you for free and open access by the Theses and Dissertations at Digital USD. It has been accepted for inclusion in Dissertations by an authorized administrator of Digital USD. For more information, please contact digital@sandiego.edu. 
UNIVERSITY OF SAN DIEGO

Hahn School of Nursing and Health Science

DOCTOR OF PHILOSOPHY IN NURSING

HISPANIC MOTHERS' NARRATIVES: EXPERIENCES OF PARENTING A CHILD

WITH CYSTIC FIBROSIS, HEALTH BELIEFS, AND HEALTH CARE

EXPERIENCES RELATED TO CYSTIC FIBROSIS

by

Linda Tirabassi-Mathis, RN; $\mathrm{PhD}(\mathrm{c})$; CPNP

A dissertation presented to the

FACULTY OF THE HAHN SCHOOL OF NURISNG AND HEALTH SCIENCE

UNIVERSITY OF SAN DIEGO

In partial fulfillment of the

requirements for the degree

DOCTOR OF PHILOSOPHY IN NURSING

\author{
$\mathrm{MAY} / 2015$ \\ Dissertation Committee \\ Jane Georges, RN, Ph.D. Chair \\ Susan Instone, RN, DNSc \\ Ann M. Mayo, RN, DNSc, FAAN
}




\begin{abstract}
The Hispanic population is the fastest growing minority in the United States and is also a minority in the cystic fibrosis (CF) community. Therefore, an increase in CF in the U.S. might be anticipated. This study explored stories of Hispanic mothers' perspectives of parenting their child with $\mathrm{CF}$, health beliefs, and health care experiences. The study interviewed 10 mothers of which half were English speaking. Narrative structural and thematic analyses were applied through a critical feminist lens. The major themes that emerged were mothering, growing and growth, mother talk, and connected; all informed by Ruddick's (1995) framework on maternal thinking. Additional major themes were life disrupted and being here. Findings were consistent with existing literature of the dominant Euro-American culture of being a mother of a child with CF. Health beliefs specific to CF care were aligned with prescribed medical treatments. Language emerged as a primary barrier for Spanish-speaking mothers. Mothers acknowledged an existing lack of awareness of CF in the Hispanic community and as underrepresented within the larger CF community. They expressed a desire to see a shift of stereotyped Caucasian images of $\mathrm{CF}$ to represent Hispanic people to promote greater awareness in the Hispanic communities and with health care providers.
\end{abstract}




\section{Dedication}

To my husband, Richard K. Mathis for his infinite support, inspiration, and love and

In loving memory of my mother, Bernetta Rose Tirabassi,

and

In honor of mothers of children with cystic fibrosis 


\section{Acknowledgment}

I am deeply grateful for the community of colleagues, family, and friends that have supported my goal from the start. It has been a long journey that has taken me to parts unknown, and brought me to discover a richer familiarity of qualitative inquiry along with much self-reflection.

First, my deep gratitude is extended to my dissertation committee, chaired by Dr. Jane Georges, in addition to Drs. Ann Mayo, and Susan Instone; who provided guidance and thought provoking discovery in the qualitative research tradition. Dr. Lois Howland whose support and dialogue helped locate my path. Thank all of you for your scholarly wisdom, direction, and coaching. I also extend great appreciation for my dear colleagues, Dr. Peggy Kalowes, who offered endless support at a click of a message; Dr. Cindy Greenberg for professional inspiration; and Julia Greenwald MSW, clinical social worker for $\mathrm{CF}$, who over the years has provided invaluable insight into perspectives of families and children with CF. I am indebted to Judy Fix whose nurse leadership has established life-long learning support to advance education and excellence in nursing. I want to especially acknowledge my interpreter, Lourdes Marquez, without her flexibility and motivation the interviews with Spanish speaking mothers would not have been realized.

I thank the eleven mothers who opened a space from their busy schedules and lives to participate in the interviews. I have gained much insight over the years from mothers who have a child with a chronic health condition, who are the voice for their child, and who have privileged me by sharing their personal stories and perspectives of their day to day lives. Likewise, the mothers in this study offered their insider views, 
willing to forge a path for others who may walk in their shoes.

Finally, I thank my family for their patience and endless support. At the inception of my studies, little did any of them know the time and energy commitment of the path I was about to embark upon. I thank and acknowledge my husband, Richard Mathis, for his care and encouragement as well as his fortitude to persevere together. I also thank and acknowledge my son, Ely Davidson, who brought his lightness of being and encouragement throughout; his significant other, Arielle Axt who is finding her path in nursing; my best friend, Kathy Bronstein whose dialogue inspires strength and balance; my sister, Leah Stringer, brother, Fred Tirabassi; Sara and Freddie Jimenez, and Dana Mathis, my stepdaughters and son-in-law, for their understanding and endurance. Collectively, I am graced with a community of family, friends, and colleagues who nurture my soul and keep me grounded. Lastly, I acknowledge my loving parents, Fred and Bernetta, who gave me the inspiration to be independent and follow my own path, and especially my mother who taught me about the ways of mothering, before I had words to know. 


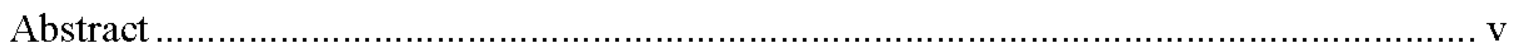

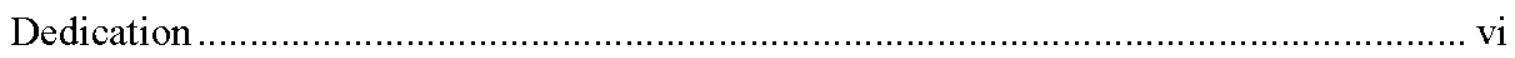

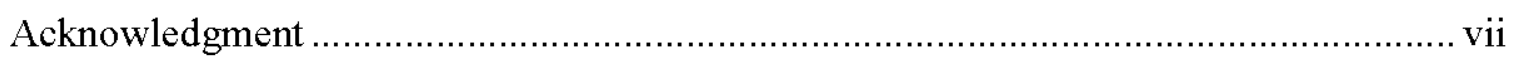

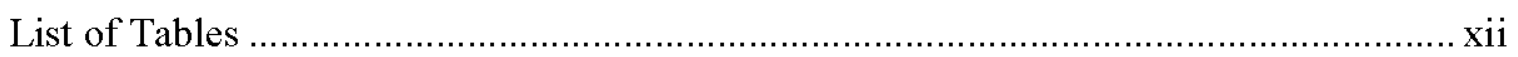

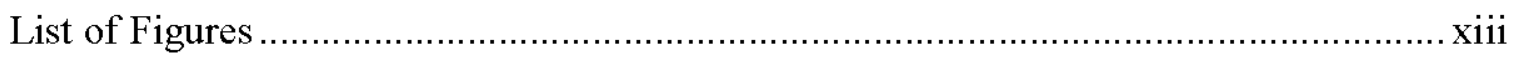

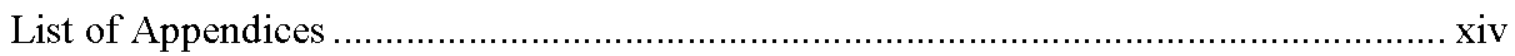

Chapter 1: Cystic Fibrosis Among Hispanics ..................................................... 1

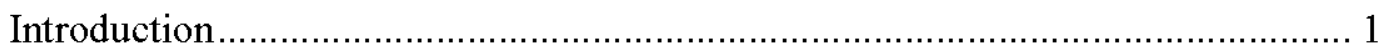

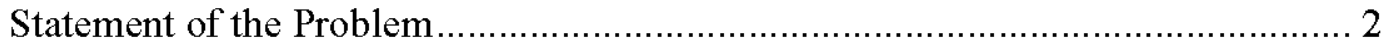

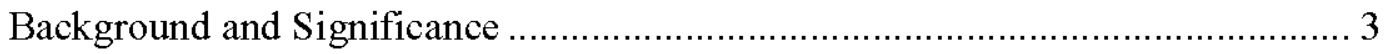

Cystic fibrosis and Hispanic ethnicity ......................................... 3

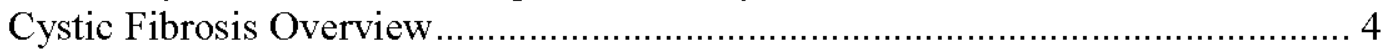

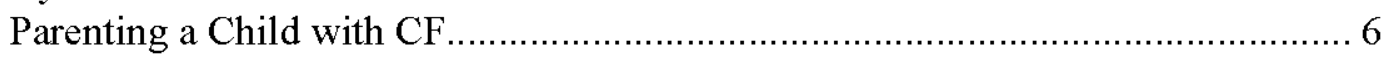

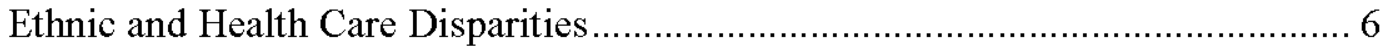

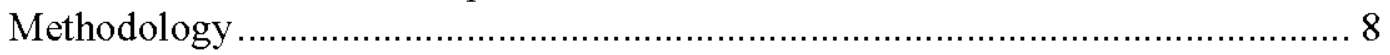

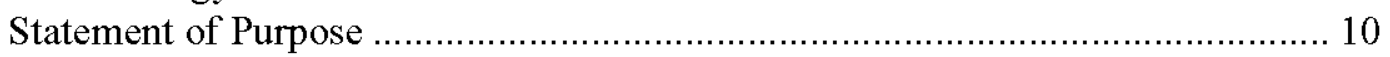

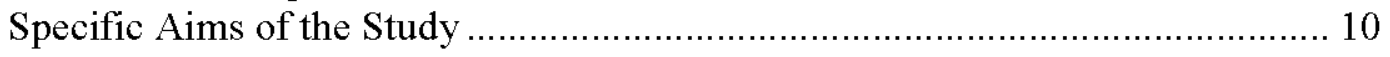

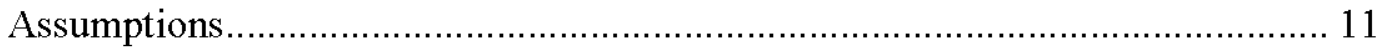

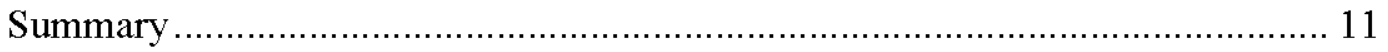

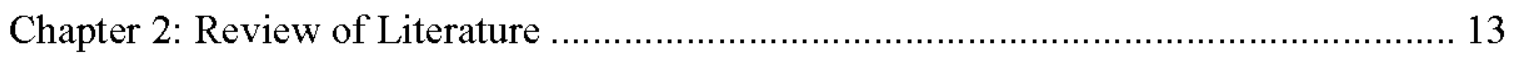

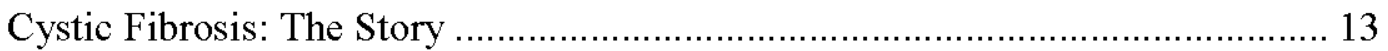

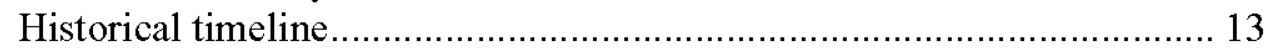

Current issues, pathophysiology, and treatments.............................. 15

Parenting a Child with CF.............................................................. 18

Parents as caregivers for children with chronic health conditions........... 18

Mothers as caregivers of children with $\mathrm{CF}$......................................... 21

The Hispanic Landscape ............................................................................ 24

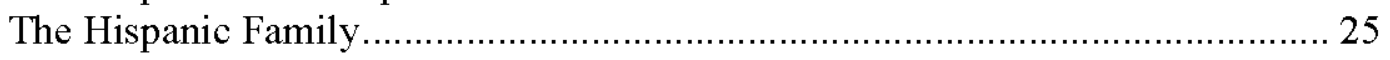

The family in the context of the Hispanic culture................................ 25

Familism, collectivism, and biculturalism .........................25 


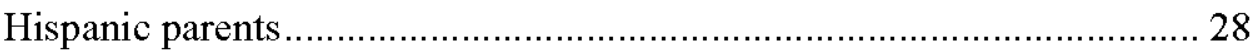

Hispanic mothers as caregivers ................................................. 29

Hispanic mothers as caregivers of children with health conditions......... 32

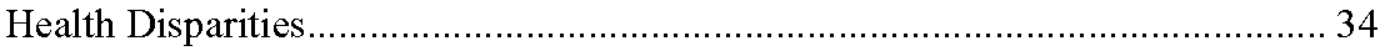

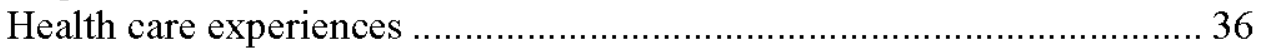

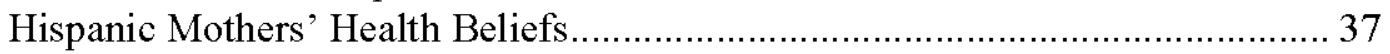

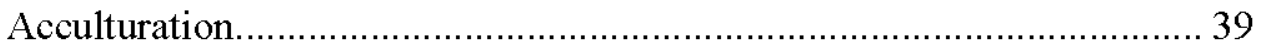

Summary of Gaps in the Literature ........................................................ 41

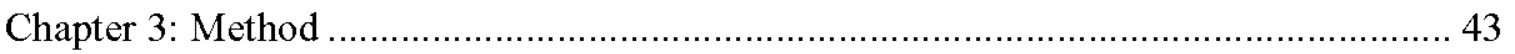

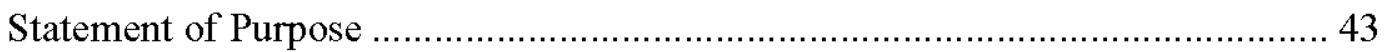

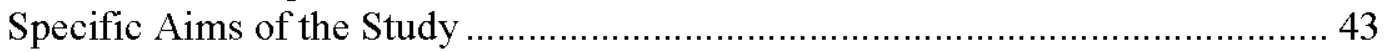

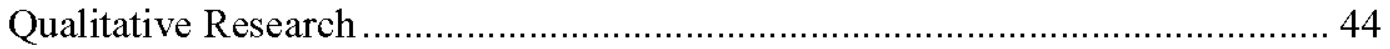

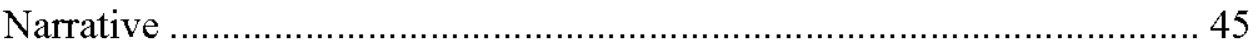

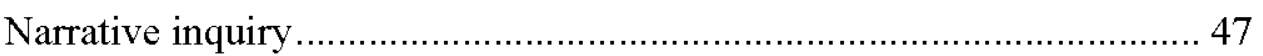

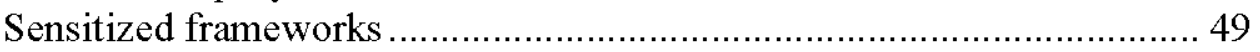

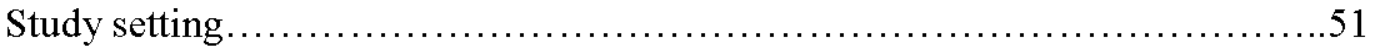

Identification and Selection of the Study's Participants .................................. 52

Human Subject and Ethics Considerations ............................................. 54

Data Collection Procedures..................................................................... 55

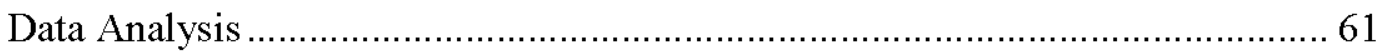

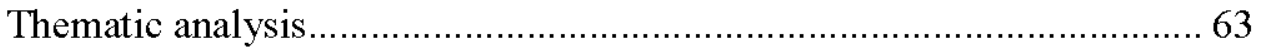

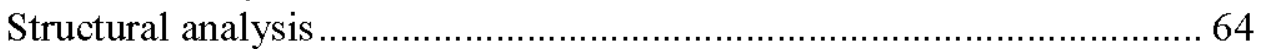

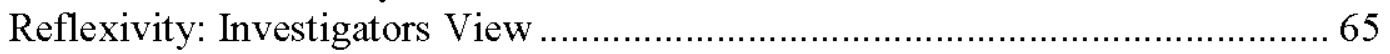

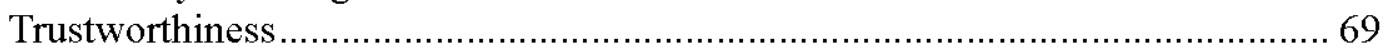

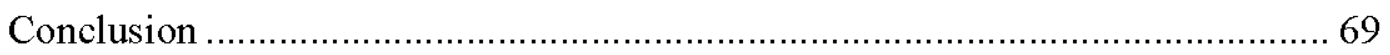

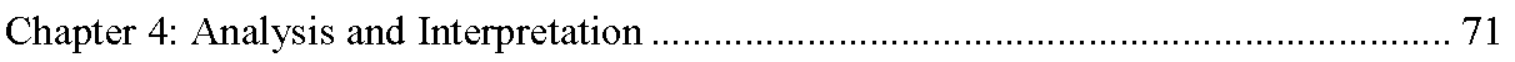

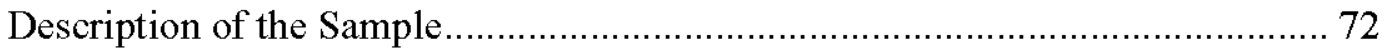

Characteristics of the mothers.................................................. 72

Characteristics of the children with CF ............................................. 74

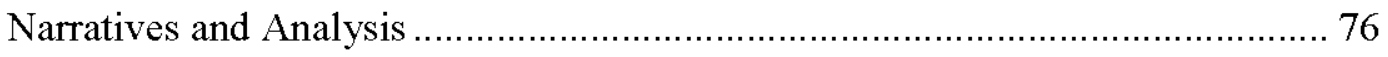

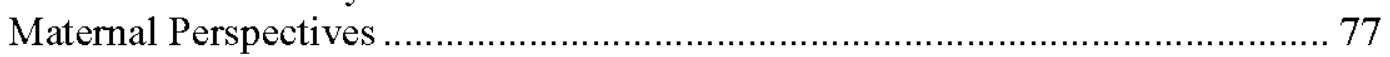

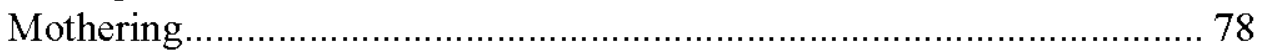

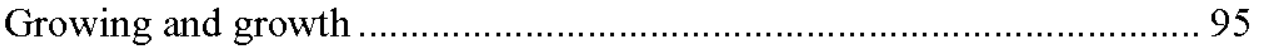

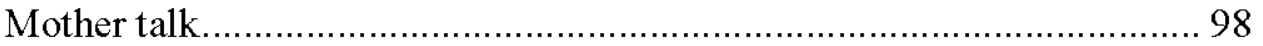

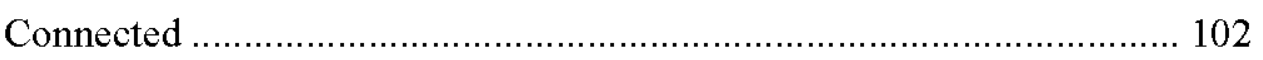

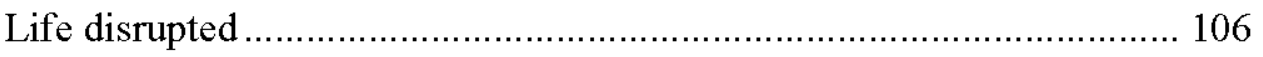




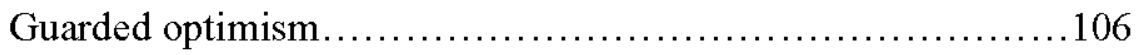

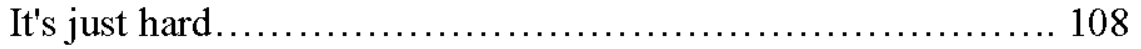

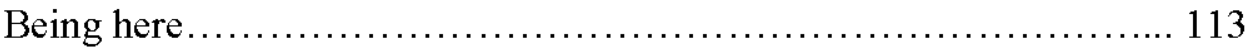

Parental gendered differences.......................... 113

More mother talk ......................................... 114

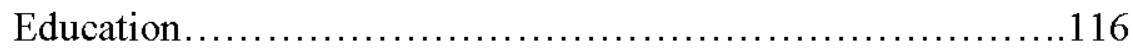

Communication won't happen without interpreters............120

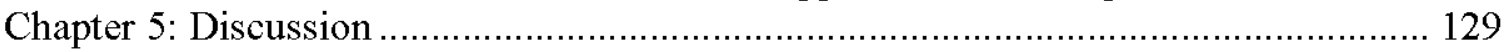

Cultural Influences on Hispanic Maternal Parenting ...................................... 133

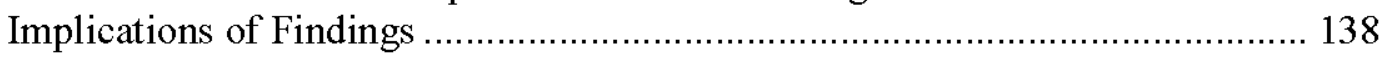

Implications for clinical practice and education ............................ 138

Future research......................................................................... 139

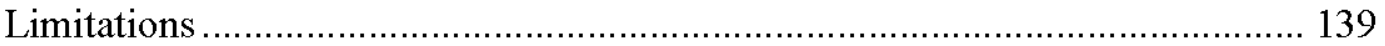

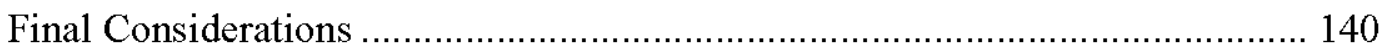

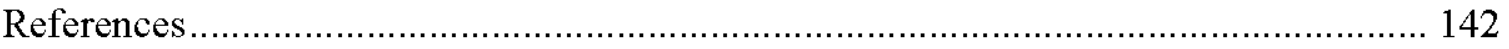


Table 1. Semistructured Interview Questions ....................................................... 56

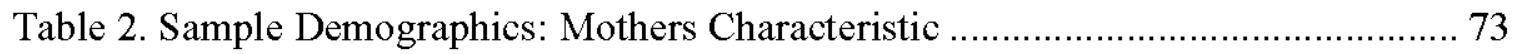

Table 3. Sample Demographics: Children Characteristic.......................................... 75

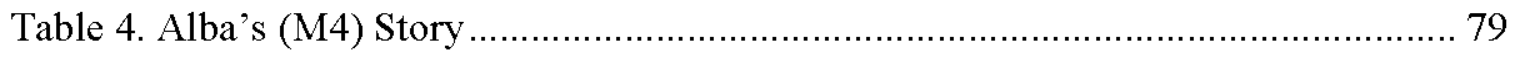

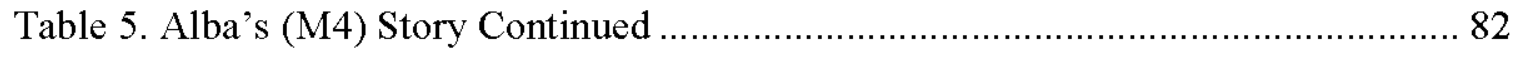

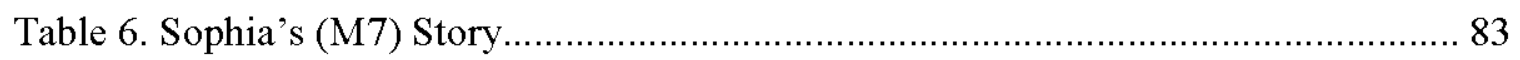

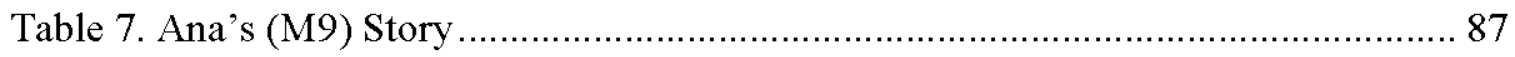

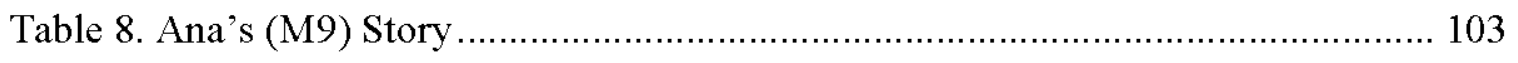

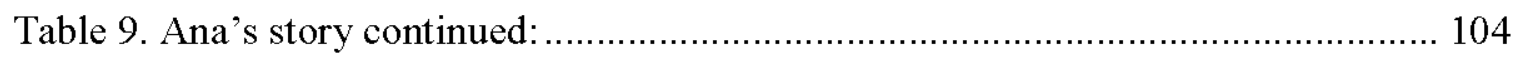

Table 10. Use of Home Remedies ................................................................ 119 


\section{List of Figures}

Page

Figure 1. Narrative and story adapted from Paley and Eva (2005) ………...................47 


\section{List of Appendices}

Appendix A: Request for Waiver of Authorization for Use and Disclosure of Protected Health Information

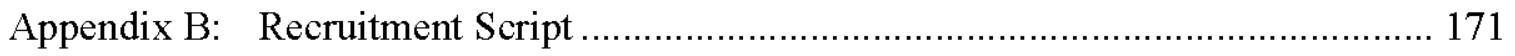

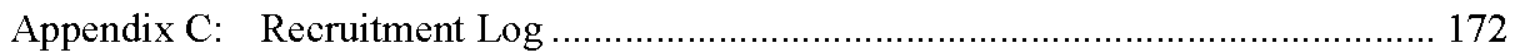

Appendix D: CF Newsletter Recruitment Announcement ................................... 173

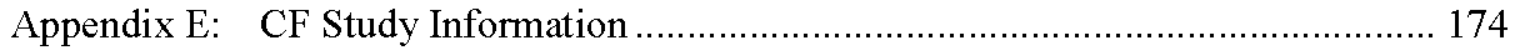

Appendix F: Informed Consent to Participate in a Research Study ........................ 175

Appendix G: Authorization for the Use of Disclosure of Protected Health Information.

Appendix H: Research Subject Reimbursement Form ..................................... 185

Appendix I: Request for Taxpayer Identification and Certification W-9 ................ 186

Appendix J: IRB Project Action Summary ..................................................... 190

Appendix K: License Terms and Conditions................................................... 191 


\section{Chapter 1: Cystic Fibrosis Among Hispanics}

\section{Introduction}

The Hispanic population is rapidly increasing as the largest minority group in the United States (Ennis, Rios-Vargas \& Albert, 2011). Genetically transmitted health conditions such as cystic fibrosis (CF) will also increase in numbers as this U.S. population increases. Although $\mathrm{CF}$ is less prevalent in the Hispanic population than in the dominant U.S. Caucasian population, it is a serious health condition commanding costly medical resources. In the U.S., CF occurs in about 1 in 4,000-10,000 Hispanic individuals compared to 1 in 2,500-3,000 Caucasian individuals (Cystic Fibrosis Foundation $[\mathrm{CFF}], 2012)$. The Cystic Fibrosis Patient Registry (CFF, 2013) reported that $7.7 \%$ of people in the U.S. with CF are of Hispanic origin, in contrast to Hispanics constituting $17 \%$ of the U.S. total population (Taylor \& Cohn, 2012).

$\mathrm{CF}$ is a genetically transmitted chronic health condition and originates from a dysfunction of a cellular protein. Individuals with CF have a variable quality of life and prognosis, largely determined by pulmonary function (Davis, 2006; Wolfenden \& Schechter, 2009). Its insidious progression eventually results in respiratory failure and death. Although primarily present in the Caucasian population, the number of Hispanic individuals with $\mathrm{CF}$ can be predicted to increase given the Hispanic population's projected rapid growth rate. In the U.S., the Hispanic population has been described as vulnerable and subject to health disparities (Berry, Bloom, Foley, \& Palfrey, 2010; Hummer \& Hamilton, 2010; Schechter, 2011). Evidence suggests that Hispanic 
individuals with CF suffer worse health outcomes and quality of life than their Caucasian counterparts, a disparity independent of genetic variation (Quittner et al., 2010; Watts, Seshadri, Sullivan, \& McColley, 2009). While ample current literature exists on CF's multiple complex aspects, research addressing Hispanic ethnicity patients with CF is limited. A recent literature search revealed no studies relative to parenting a child with CF through the lens of Hispanic mothers. This study explores the experiences and perceptions of Hispanic mothers parenting a child with CF. It also explores maternal perspectives of health beliefs and health-care experiences related to their child's care with CF. This information is critical to culturally congruent intervention's future development, targeted health promotion, and for patient-centered management provisions of Hispanic individuals with $\mathrm{CF}$ and their families.

\section{Statement of the Problem}

As the Hispanic population increases, it follows that Hispanic-origin individuals diagnosed with CF will also increase commensurate with their population growth. Epidemiologic data report that Hispanic individuals with CF experience a significantly greater decline in their pulmonary function than their Caucasian counterparts (Schechter, 2011; Watts et al. 2009); this finding has not been fully explained. Many studies describe parent experiences of living with a child with CF (Carpenter \& Narsavage, 2004;

Gjengedal, Rustoen, Wahl, \& Hanestad, 2003; Glasscoe \& Smith, 2011; Jessup \& Parkinson, 2010; Moola, 2012; Szyndler, Towns, van Asperen, \& McKay, 2005; Williams, Corlett, Dowell, Coyle, \& Mukhopadhyay, 2009). Studies have also explored mothers' specific concerns as caregivers of their child with CF (Gayer \& Ganong, 2006; Hodgkinson \& Lester, 2002; Yilmaz et al., 2008). However, review of the literature on 
Hispanic mothers' experiences parenting a child with CF revealed an unexplored area. This population has been underrepresented in studies related to the $\mathrm{CF}$ experience. Therefore, this qualitative research study explores maternal perspectives and experiences of parenting a child with CF through the lens of Hispanic mothers. The study also explores maternal perspectives of health beliefs and health-care experiences related to their child with CF. Qualitative research findings inform nursing practice on the subtleties and complexities of the human response to health and illness, essential to the development of effective and culturally congruent interventions (Sandelowski \& Barroso, 2003). Qualitative research illuminates intricacies of human behaviors that might not be fully uncovered through reductionist approaches of quantitative research.

\section{Background and Significance}

Cystic fibrosis and Hispanic ethnicity. The estimated prevalence of CF in the Hispanic population ranges from 1 in 9,200 to 1 in 13,500 (Rohlfs et al., 2011). U.S. Hispanic individuals' with CF demographic trend has continued to increase from $4.1 \%$ in 1992 , to $5.7 \%$ in 2002 , and most recently to $7.7 \%$ in 2012 (CFF, 2013). It is anticipated that Hispanic individuals with $\mathrm{CF}$ will continue to increase, not only proportionate with the increasing Hispanic population, as the fastest growing minority in the U.S., but also through increased identification from mandated statewide newborn screening. More specifically, the overall Hispanic population growth reported in the 2010 U.S. Census (as cited in Ennis et al., 2011) was 43\%, four times that of the growth in the general population. Based on this data, one may surmise the Hispanic group, considered a minority group in the U.S., is also a minority among the CF community. 


\section{Cystic Fibrosis Overview}

An estimated 10 million CF genetic carriers reside in the U.S. (CFF, 2012); approximately 1 in 29 people are carriers in Caucasians and 1 in 46 people are carriers in the Hispanic population, representing the two highest carrier frequencies. Genetic research has exponentially expanded the understanding of CF and continues to contribute data on mutations that affect aspects of $\mathrm{CF}$ and its care. A variety of disciplines have contributed to these scientific advances directed toward increased survival rates. While hope and optimism surround increased survival, psycho-social difficulties compound the physiological complications as individuals grow older with $\mathrm{CF}$.

$\mathrm{CF}$ is classified as one of the most common life-shortening genetically transmitted conditions of children. Approximately 30,000 individuals are in the U.S.; those older than 18 years are approaching $50 \%(\mathrm{CFF}, 2014)$. The median survival age is reported to be in the early fourth decade for an infant born today. Although this population is relatively small, its health care reflects costly resource utilization. Commercially insured patients with $\mathrm{CF}$ averaged annual medical costs 15 times higher than the average commercially insured member: $\$ 64,000$ compared to $\$ 4,300$ (J. Ranville, personal communication, September 20, 2013). O’Sullivan, Sullivan, Higuchi, and Montgomery (2011) examined Medicaid and commercial enrollee's health care claims for both children and adults with $\mathrm{CF}$ for both medical and pharmacy utilization. Findings indicated the mean annual cost combined for medical care and pharmaceutical agents approached $\$ 50,000$, excluding out-of-pocket expenses.

$\mathrm{CF}$ is a chronic, complex, multisystem condition with variable severity characterized by a slow decline in lung function, resulting in eventual respiratory failure. 
Individuals live between episodes of relative wellness and exacerbations most commonly caused by acute pulmonary infections. Because of demanding care requirements, it has been described as an intrusion into day-to-day life (Bluebond-Langner, 1996). Its chronic, progressive nature and multisystem involvement positions it as a prototype for chronic conditions in children (Admi, 1996). Despite scientific advances, there is no cure for this, which progresses to either lung transplant as a life-extending option, or eventual death.

The explosion of genetic information that advanced the understanding of CF had its inception in 1989, when the CF chromosome was identified (Rommens et al. 1989). Prior to this landmark discovery, CF was infrequently considered in ethnic or racial groups other than Caucasians, except in accredited CFF centers. Accreditation through the CFF certifies CF centers provide a standard of care by highly specialized professionals. Thus, the complexity of CF mutations has expanded its footprint to both African Americans and Asians as well as Hispanic ethnicities, extended through the science of genetics.

It is incumbent upon the health care community to understand better the experiences of Hispanic mothers' who are parenting a child with $\mathrm{CF}$, and to increase understanding of their health care perspectives. Overall, California is the most populated state in the U.S. in which people reside with CF (CFF, 2013), and it has the highest concentration of people of Hispanic heritage (Ennis et al. 2011). Thus, the increasing numbers of individuals with CF surviving into adult years, coupled with the increasing overall rise of the Hispanic population, and the noteworthy poor prognosis, lend significant relevance to understanding maternal perceptions and experiences of parenting a child living with $\mathrm{CF}$. 


\section{Parenting a Child with CF}

One function of family nursing is to understand the family and family members' experiences and responses to health and illness. While CF has been extensively studied, there is a dearth of information about Hispanic families and family members with CF. Specifically, experiences, health beliefs, and health practices of Hispanic families living with a child with $\mathrm{CF}$ have not been described. While valuable contributions from genetics and epidemiology have advanced science, more information is needed to appreciate the strengths and challenges influencing Hispanics. While nursing practice has embraced culturally sensitive and patient- and family-centered care, integration of these concepts might be less evident to meet the pressing health care needs of the nondominant Hispanic group with CF. Socioeconomic and cultural contexts shape the family's lives, parenting, and health care practices. It is timely for nurses and health care providers to attend to demographic, ethnic, and cultural vulnerabilities of families and extend their reflection to relationships of health care organizations, families, and their own professional practice.

\section{Ethnic and Health Care Disparities}

Disparities in health's social determinants are a national health agenda for Healthy People 2020 objective (2010 Health disparities are defined as differences in the quality of care received by minority and nonminority groups that have equal care access (Smedley, Stith, Nelson, \& Committee on Understanding and Eliminating Racial and Ethnic Disparities in Health Care, 2012a) and have been extensively documented (Brookins, 1993; Flaskerud et al., 2002; Flores and The Committee on Pediatric Research, 2010; Friedman, Bowden, \& Jones, 2003; Newacheck, Stoddard, \& McManus, 1993; Zambrana \& Dorrington, 1998). The Institute of Medicine's landmark report, 
Unequal Treatment: Confronting Racial and Ethnic Disparity in Health Care (as cited in Smedley et al. 2002a) concluded that health disparities exist in minority patients and described the complexities that have resulted in discordant health care experiences of vulnerable groups. The report identified three primary contributors to health care disparities: (a) "organization and operations of health care systems," (b) "patients' attitudes and behaviors," and (c) "biases, prejudices, and uncertainties from health care providers during clinical encounters" (p. 2). While the report acknowledged that stereotyping has been shown through social psychology research to be an almost universal cognitive human application that provides a means to organize or describe information about people and groups, it also acknowledged the downside of stereotyping: that is by its very nature, stereotyping leads to unfairness and bias. According to the report, health care providers were influenced by a client's race or ethnicity and associated stereotypes. An increased awareness of health disparities, education, and cultural competencies resulted. However, a more recent report documented health care practices continue to undermine individuals and contribute to pervasive marginalization of people from different ethnicities and minorities (Flores and The Committee on Pediatric Research, 2010). These people are too frequently associated with less socioeconomic, education, and resource capital. According to Freire (1993), who espoused social change for the oppressed, asserted that people who are "welfare recipients" (p. 74) are treated as those who deviate from the good, organized, and just society and "regarded as pathology of the healthy society" (p.74). In order to provide equitable quality care and reduce disparities, continued research is needed to understand the concerns, experiences, and needs of underserved and marginalized cultural and ethnic groups. 


\section{Methodology}

A qualitative methodology informed this study and explored the experiences of Hispanic mothers who parent a child with CF. Specifically, narrative research, as a method, begins with experiences as represented in the storied lives of individuals and groups (Bloomberg \& Volpe, 2012). It provided an in-depth exploration of Hispanic maternal perspectives of parenting a child with $\mathrm{CF}$, their health beliefs, and experiences related to $\mathrm{CF}$ care that have been overlooked and taken for granted. This method of inquiry is oriented from a naturalistic stance that directs studies in the most natural state, yielding rich descriptions of the experiences told directly by mothers. Personal narratives, stories told by mothers on their everyday life experiences or that reflect a significant life event (Langellier, 1989) help to understand how people make meaning out of events in their lives (Chase, 2005, p. 660). Chase offered insight into the relationship of the researcher with the interviewee; the researcher makes a "conceptual shift toward the idea that an interviewee has stories to tell and a voice of their own" (p. 660), narrating their own story, in contrast to the interviewee having answers to the research questions of interest. Narrative research is most often referred to as narrative inquiry; thus, will be the term used in this study. It was appropriate for this study, as a preliminary step, to describe experiences not yet documented. This approach also allows for accumulated details from personal stories that are then able to be assembled in a "fuller picture of the individual or group" (Riessman, 2008, p. 11).

This study was sensitized by critical social theory and feminist theory. Critical social theory has its roots in the social sciences and pertains to social processes, relationships, structures, and philosophy. It is relevant to health care, as it relates to 
empowering patients and families to participate freely in their own care and decisions and to make changes by taking control of their lives (Allen, 1987; Holloway \& Wheeler, 2010). From a critical theoretical standpoint, health care institutions and health care providers might unwittingly communicate a culture of imposed constraints, biases, and directives to their patients and families, thus, restricting space for perceived partnered or participatory relationships. The critical and feminist paradigms are particularly appropriate as philosophical underpinnings for this study, as voices from underserved groups have been underrepresented and marginalized in research. Narrative methodology, critical social theory and feminist theory are discussed further in Chapter 3.

Family and providers of health care research was described as an emerging area of interest for theory, research, and clinical interventions as recently as the 1980 's (Doherty \& McCubbin, 1985). Family studies related to health care and chronic conditions have flourished and continue to inform approaches for best care derived from its dynamic nature and influenced by behavioral, biomedical, and economic forces. In addition, with the evolving changes among families and chronic health conditions, the intersections of many disciplines have cross-pollinated family studies. Thus, reviewed studies for this project originated from not only nursing, but also from medicine, psychology, and sociology. These disciplines reflect the interrelatedness of the complex nature of chronic health conditions, such as $\mathrm{CF}$, and the professionals that form specialty teams to manage these conditions.

This study was an initial step in filling an existing gap in the description of mothers' experiences parenting their child with $\mathrm{CF}$ from a Hispanic perspective. It adds to the advancement of nursing science by contributing to a greater understanding of 
maternal perspectives of parenting a child with CF through a lens culturally different from the dominant Euro-American representation largely found in the literature. It augments the understanding of maternal health care beliefs, practices, and experiences within their social and cultural relationships to the health care system and providers. Uncovering this population's unique needs for future interventions to improve patient outcomes and reduce health care discordance is timely and past due.

\section{Statement of Purpose}

The purpose of this study was to explore Hispanic mothers' perceptions parenting a child with $\mathrm{CF}$ and maternal perspectives of their health beliefs and health care experiences related to CF. The goal of the study was to describe Hispanic mothers' parenting experiences who have a child with $\mathrm{CF}$ to further the understanding and eventual development of culturally sensitive and culturally congruent interventions to improve patient care and outcomes. This study's specific research question asks what it is like for mothers parenting a child with $\mathrm{CF}$ from a Hispanic lens within their sociocultural context. In addition, this study addresses the following question: What are the maternal health beliefs and health care experiences for their child with CF from an ethnic Hispanic perspective?

\section{Specific Aims of the Study}

The specific aims of the study were to:

- Aim 1: Explore and describe Hispanic maternal perspectives and experiences of parenting a child with $\mathrm{CF}$.

- Aim 2: Explore and describe the health beliefs from Hispanic maternal perspectives related to health care of their child with $\mathrm{CF}$. 
- Aim 3: Explore and describe health care experiences from Hispanic maternal perspectives related to care of their child with $\mathrm{CF}$.

For the purpose of this study, the term Hispanic was used to represent both Hispanic and Latino ethnicity. When descriptor terms other than Hispanic are used, it reflects the specific group referenced in the cited study.

\section{Assumptions}

1. Personal stories reflect the daily life of the story tellers in their (re)constructed reality.

2. CF as a complex, life-shortening condition has universal meaning and is also based on its ascribed meaning by the family and mother.

3. Health care beliefs and practices might influence home care treatments of children with $\mathrm{CF}$ in the Hispanic cultural context.

4. Power relations, rooted from dominant Euro-American paternalism, continue to exist in families, the health care environment, and society.

5. Stereotypical and traditional gender roles are reinforced when mothers of chronically ill children, such as with $\mathrm{CF}$, are primary caregivers.

\section{Summary}

$\mathrm{CF}$ is a rare yet complex, costly, condition that is "aging out" or making the transition to adult care from pediatric care because of improved prognoses. Scientific advances have further established its panethnic influence. Hispanic heritage individuals are increasing in the U.S. population and are also increasing in the CF population, creating a double jeopardy: first from an ethnic vulnerability and second, from a CF diagnosis, found primarily in the dominant Euro-American heritage. It is incumbent upon 
the health care and the CF communities to learn and understand the perspectives of Hispanic heritage mothers to gain culturally sensitive views toward improving patient outcomes through partnerships with this fastest growing minority population. They have not been heard; it is time. 


\section{Chapter 2: Review of Literature}

Chapter 1 presented the study's background and significance related to exploring Hispanic mothers' perceptions of having a child with CF. Literature and the state of science as they relate to phenomena central to the study aims are reviewed in this chapter. The review of literature is focused on the following areas: (a) an overview of CF and management, (b) parenting a child with $\mathrm{CF}$, (c) the Hispanic landscape, (d) Hispanic family, (e) ethnic and health disparities, (f) health care experiences, (g) Hispanic health beliefs, (h) acculturation, and (i) the investigator's reflexive view. This study's fundamental aim explores and describes Hispanic maternal perspectives and experiences of parenting a child with CF and explored Hispanic mothers' perceptions of health beliefs and health care experiences related to $\mathrm{CF}$.

\section{Cystic Fibrosis: The Story}

Historical timeline. In order to understand CF's current state of science, a review of its historical context is presented. CF has an intriguing beginning traced back to the 16th century. Folklore of the era indicated that parents were cautioned to beware of early infant death when their lips tasted salty after a kiss to their infant's brow (Quinton, 1999). This observation is acknowledged as a hallmark of CF because of the concentrated sodium and chloride found in sweat. CF, as a disease, was first identified in 1938 by Dr. Dorothy Andersen from a series of postmortem infant examinations. A pattern of malnutrition and obstructive mucus collections noted in multiple mucus secreting glands was described (Davis, 2006). Pathology of the pancreas with growth failure and 
malabsorption of fats and proteins was thought to cause weakening of the respiratory system, leading to infection and eventual death. The characteristic description of tenacious thick mucus in the exocrine glands distinguished $\mathrm{CF}$ from other conditions that shared symptomatology. In 1948, Dr. Paul di Sant'Agnese made a landmark discovery following a massive heat wave in New York and across the country (Davis, 2006; Quinton, 1999). Numerous infants presented at the children's hospital with heat prostration. di Sant'Agnese hypothesized that these infants had abnormal sweat. This work demonstrated a five-fold elevation of sodium and chloride in the infants whose electrolytes remained elevated after the heat wave. This work paved the way for the development of the sweat test that continues to be the diagnostic gold standard (Farrell et al. 2008). Credibility was gained from the early observations of the 16th century.

Throughout the decades, much of the research has been devoted to understand the origin of CF. Quinton, a researcher, who has $\mathrm{CF}$, built upon the existing research that identified CF's basic electrolyte defect. He extended the research by identifying that the basic defect in the CF duct was related to anion impermeability with sodium and chloride versus a defect of anion exchange (Davis, 2006). Greater understanding of these mechanisms along with simultaneous research explored and propelled better treatments. International and national foundations were established in the 1950s and 1960 s to share findings and advance CF's science and treatment of. In 1989, chromosome 7 was identified as the genetic basis for CF (Corbyn, 2012; Rommens et al. 1989; Thursfield \& Davies, 2012). This landmark discovery offered great hope and optimism that a cure would be forthcoming; however, the reality was quite different. There are more than 1,800 genetic mutations (CFF, 2013) associated with $\mathrm{CF}$, contributing to the complexity 
for discovering the cure. DNA mutation testing supplements the sweat test for identification of specific CF mutations. DeltaF508 is the most common mutation accounting for $90 \%$ of the $\mathrm{CF}$ population worldwide carrying at least one copy (Corbyn, 2012; Rommens et al., 1989; Thursfield \& Davies, 2012).

Additional critical dates in CF history are the first heart-double lung transplant in 1984, followed with double lung transplant in 1988 (Kotloff \& Zuckerman, 1996). Subsequent success of a double lung with domino heart transplant was celebrated followed by living related-donor double lung transplant (Littlewood, 2011). With advances in treatments and pharmacologic agents, more effective and efficient treatment regimens have improved the prognosis.

As noted in Chapter $1, \mathrm{CF}$ is an autosomal recessive condition occurring with greatest prevalence in Caucasians. An extensive history that Littlewood (2011) detailed identified four novel minority CF cases with: (a) an African American in 1962, (b) two American Indians in 1968, and (c) a 1968 report of a child in India, where CF was thought to be nonexistent. The historical timeline also described CF occurring in Japanese and Chinese ethnicities about the same time. Interestingly, while both race and ethnicity were described outside of Caucasians in the historical timeline, there is a notable absence of reference to Hispanic ethnicity. While the human genome project has advanced the understanding of CF exponentially, less attention has been directed toward understanding experiences of minority groups living with CF in the U.S.

Current issues, pathophysiology, and treatments. More recently, the basic defect in the CF gene has been further defined as an altered function of the $\mathrm{CF}$ transmembrane regulator (CFTR) protein. The CFTR protein controls sodium, chloride, 
and water's movement across the cell membrane; CFTR dysfunction causes reduced movement of these elements across the cell membrane resulting in the classic thick mucus associated with CF (Derichs, 2013). CF is divided into six different CFTR dysfunction classes (Derichs, 2013; Wolfenden \& Schechter, 2009). Class-specific therapy is underway toward reaching the cure. The most exciting current scientific breakthrough is the development and Federal Drug Administration approval of a classspecific drug, Ivacaftor, in April 2012 (Corbyn, 2012; Derichs, 2013). Normalization of sodium and chloride movement at the cell wall surface with Ivacaftor was reported. Because of its less frequent genotype, about $4 \%$ of people with CF will benefit from this treatment, estimated to cost upward of $\$ 294,000$ per person annually (Corbyn, 2012). Significant patient outcomes from the double-blind study demonstrated weight gain and normalization of sweat chloride. This marks the first therapy designed to treat the cause of $\mathrm{CF}$ rather than to mitigate symptoms. Application of this drug combined with existing drugs to attack the different classes of CFTR dysfunction are currently in the pipeline. These new drugs have catalyzed renewed hope and optimism in the CF community and health care providers. Increased understanding of $\mathrm{CF}$ has progressed a long way from the tales of folklore, and the expected median predicted age of survival for an infant is reported to be in the early 40 s with $49 \%$ older than 18 years of age (CFF, 2014).

$\mathrm{CF}$ is classified as a life-shortening condition, despite its recommended day-today care. It is characterized by declining pulmonary function, pancreatic insufficiency, malabsorption, poor linear growth, and delayed onset of puberty. In addition, many individuals with $\mathrm{CF}$ suffer from pan-sinusitis, recurrent pulmonary infections, and a persistent cough. While the cough mobilizes thick secretions, it is a hallmark of $\mathrm{CF}$ and 
might also contribute to social stigma. As a result of living longer, various complications could be encountered, challenging daily care. Complications, such as CF-related diabetes, with an onset as early as adolescence, hemoptysis, and chronic headaches are examples of burdensome complications. Missed school days become a concern as a result of a pattern of exacerbations and, so called remissions, commonly associated with the cycles of chronic illness. There is still no cure for CF. Lung transplant is the only option to extend life. It remains a deadly disease, despite great strides in scientific progress and life- extending options. Individuals living with $\mathrm{CF}$ wake each day to all-consuming treatment regimens with hope and optimism that a cure will be announced soon (Jain \& Goss, 2014; Jamieson et al., 2014)

Nonetheless, the daily management and care is intrusive and burdensome. The home-care regimen is time intensive and intensifies with pulmonary exacerbations accompanied with a hyper-metabolic state and fatigue. Typical daily care usually includes two to four separately administered nebulized medications with a minimum of two airway clearance techniques demanding about 45 minutes in the morning and at bedtime for basic health maintenance. In addition, multiple nutritional supplements, oral vitamins, and replacement pancreatic enzymes taken with every meal and snacks are prescribed to replace what the body does not produce. Attention to a caloric dense, highprotein, high-fat nutrition plan is recommended, not only for optimum growth and development, but also to optimize lung health. A positive correlation between nutrition and lung health has been reported (Milla, 2007).

The intrusion of daily care is a challenge for most individuals with $\mathrm{CF}$. The strengths and vulnerabilities of individuals living with CF, including their families' 
experiences, are illuminated through their personal stories described in numerous studies (Bluebond-Langer, 1996; Carpenter \& Narsavage, 2004; Christian \& D'Auria, 1997;

George et al., 2010; Gjengedal et al., 2003; Jessup \& Parkinson, 2010; M. McCubbin, Bowers, \& Holaday, 1984; Moola, 2012; Szyndler et al., 2005; Tracy, 1997; Williams et al., 2009). However, these studies make no reference to the participants' ethnicity; therefore, the assumption that Caucasians are the sole study participants is presumed because of their dominant prevalence and absent participant ethnic demographics. While these studies provided an emic view of living with $\mathrm{CF}$, they also underscored the gap that exists about what is known, or not known, about Hispanic individuals' experiences with $\mathrm{CF}$ and their families.

\section{Parenting a Child with $\mathrm{CF}$}

Parents as caregivers for child ren with chronic health conditions. Admi's (1996) narrative of an ordinary lifestyle suggested that outsiders might view the centrality of a chronic condition with more gravity than those actually living with the chronic health condition. While this is an authentic finding from narratives of young mothers of the study, it might not resonate with parents whose children have chronic conditions. A parent's role in contemporary society is challenged by the shear nature of stimuli, demands, and juggling daily schedules. When the parent role is superimposed over the caregiver of a child with a chronic health condition role, the responsibilities expand exponentially. The parent role does not come with a job description. Sullivan-Bolyai, Sadler, Knafl, and Gilliss (2003) described direct and indirect caregiving tasks deemed as overarching categories of responsibility when parenting a child with a chronic health condition. Four categories of parent's major responsibility were: (a) "managing the health 
condition, (b) caring for self," (p. 6) (c) "functioning as the child's case manager, and (d) maintaining the family unit" (p. 7). Subsequently a position description, although not exhaustive, was developed (Sullivan-Bolyai, Knafl, Sadler, \& Gilliss, 2004) to illuminate health care providers on the complexities required of day to day management of caring for a child's chronic health condition. Critical parenting attributes were further described from a concept analysis as nurturance, responsibility, and guidance (Virasiri, Yunibhand, \& Chaiyawat, 2011). While caring for a child with a chronic health condition demands much attention and time of the parenting role, it was also found be a most overwhelming task, resulting in fatigue, role strain, and depression. Characteristics such as grief, fear, vigilance, isolation, confusion, a fragile sense of control, advocacy, and uncertainty were described in two metasyntheses of parenting a child across a spectrum of chronic health conditions (J. S. Coffey, 2006; Kepreotes, Keatinge, \& Stone, 2010). In addition, these studies reported more covert findings that many families eventually defined as a new normal. Families tried to make meaning of their lives, find elements of strength, and construct positive associations such as strengthening their marriage or families, redefining their role of mothering, and having hope.

CF has been described as a prototype of chronic illnesses owing to its multisystem, complex care and management. It has been studied from multiple perspectives in an attempt to augment a better understanding and to support the needs of the family, caregivers, and the affected child. The literature reflects mixed findings when describing parents' and mothers' experiences as caregivers of their chronically ill child. In part, findings reflect inconsistent definitions of terminology, roles, functions, and contexts (Gage, Everett, \& Bullock, 2006; Nicholl \& Begley, 2012). Studies have 
explored parenting a child with a chronic health condition through a general perspective on chronic illness (J. S. Coffey, 2006; Kepreotes et al., 2010; Ray, 2002), disease-specific perspectives (Carpenter \& Narsavage, 2004; Cronin, 2004; Moola, 2012), and other factors such as coping and adjustment (Rehm, 2000), family function (Knafl \& Deatrick, 1986; McClellan \& Cohen, 2007); sleep (Meltzer \& Mindell, 2006; Yilmaz et al., 2008), and depression (Besier et al., 2011; Smith, Modi, Quittner, \& Wood, 2010). Parenting a child with a chronic health condition has also provoked parenting studies, particularly the traditional maternal role as primary caregivers. A review of literature related to parents and mothers of children with chronic health conditions follows.

Two meta-syntheses captured salient pervasive findings from the literature (J. S. Coffey, 2006; Kepreotes et al., 2010). J. S. Coffey (2006) reported findings from 11 studies on parenting a child with a chronic illness. Seven themes emerged. Overall, the mothers reported carrying the burden of care for their child. The most relevant findings described themes that reinforced the primary maternal role as a constant experience for the child and a heightened worry above typical parental worry. For example, worry was described spanning from the time surrounding the diagnosis to future oriented, such as worrying about who will care for their child as the parent ages. Depression and exhaustion also surfaced consistent with findings from reviewed studies. Similarly, Kepreotes et al. (2010) analyzed 10 studies to determine whether experiences of parenting a child with a chronic condition had changed within a more contemporary time frame from 2000-2009. Findings reflected some overlapping of themes with J. S. Coffey's, but also some differences. Kepreotes et al. discussed immediate grief after the diagnosis and ongoing grief as a major theme, as well as deficits of information, and 
inadequacy of some relationships from health care providers. For example, as their child's primary caregivers, the parents, as the child's advocate, expected their voices would be respected by their child's health care providers. When the parents' experiences were less than their expectations, they felt restricted to assert their advocacy role for fear their child's care might be put at risk. Ray (2002) studied 30 mothers and 13 fathers to make the invisible work of parenting their child with a chronic health condition tangible. In addition to similar themes in the aforementioned studies, topics such as exhaustion, feeling overwhelmed, isolation, and vigilance were also described embedded in the invisible work of parents. These findings are consistent with J. S. Coffey and Kepreotes et al. Invisible work was deconstructed as "monitoring physiologic functions, anticipating the need for adaptive strategies, facilitating and monitoring the child's success in social situations, and working with health, education, and social sectors" (Ray, 2002, p. 435) The invisible political and advocacy work added to their caregiving roles yet put them at risk for greater demands, leading to exhaustion and role overload (Ray, 2002). Lindström, Aman, and Norberg (2010) found increased prevalence of burnout symptoms in parents of children with diabetes and inflammatory bowel disease when compared to healthy controls; the highest reports were from mothers of children with diabetes. Mothers, as caregivers, included other roles such as decision makers, educators, and counselors. These roles have consistently been attributed to the primary caregiver role (Friedman et al., 2003; Gage et al., 2006; Gibson, 1999). Mothers as caregivers are discussed next.

Mothers as caregivers of children with CF. Approaches to understanding the care required of chronic health conditions from studies of both generic and disease specific, such as $\mathrm{CF}$, further support the maternal role as primary caregivers. Hodgkinson 
and Lester (2002) explored stresses, coping strategies, and nurse support strategies for mothers with a child with CF. Responses from 17 mothers reported their highest stressors as: feeling in the middle with health care decision making, particularly related to genetic testing; feeling burdened with the responsibility of daily care; isolation; and losing one's sense of identity because of caregiving demands. Coping strategies underscored support, particularly with their nurse relationships. These findings are consistent with other studies depicting caregivers' reports of CF (Gayer \& Ganong, 2006; Gibson, 1988; Glasscoe \& Smith, 2011; Götz \& Götz, 2000; Hunter, 2003; M. McCubbin et al., 1984), intertwined with additional themes such as living with uncertainty (Glasscoe \& Smith, 2011; Hunter, 2003), normalization (Cronin, 2004; Glasscoe \& Smith, 2011; Knafl \& Deatrick, 1986; Knafl, Deatrick, \& Havill, 2012; Moola, 2012), and living day to day (Glasscoe \& Smith, 2011; Moola, 2012). Some study findings differed relative to comparison groups' backdrops. Cronin compared mothering children who had hidden impairments, attention deficit hyperactivity disorder (ADHD), and CF, while Moola contrasted parenting children with CF to those with congenital heart disease. Cronin's findings revealed distinct differences in mothering between ADHD and CF. Specifically, support and exhaustion were dominant with ADHD, contrasted with normalization as dominant with CF. Even though fatigue among caregivers of CF has been reported (Ray, 2002), fatigue's nature of might be contextually different. Moola's (2012) study described similarities between narratives of congenital heart disease and CF caregivers. Both groups described stress; however, stress reported by parents' of children with congenital heart disease was time limited in contrast to $\mathrm{CF}$, where unresolved and ongoing stress was reported. Both groups conveyed a similar coping strategy through gaining insight 
that aided their illness perspectives, such as comparing their child's condition with others who were perceived as more severe). Finally, the CF group differed in what was termed "stolen time" (p. 218), as narratives described time dominated by daily treatments.

Wong and Heriot (2007) studied parent coping strategies in relation to child and parent adjustment with vicarious hope in attempts to understand better parent and child adjustment variances with CF. Vicarious hope, described as "parental expectations that desirable things will happen in their child's future" (p. 344), was contrasted with vicarious despair, described as "parental expectations that undesirable things will occur in their child's future" (p. 344). Findings indicated that both constructs are distinctly different from coping. Findings further indicated that the mental health of children with CF can be strengthened by enhancing parental vicarious hope and reducing self-blame. Parents who experienced greater vicarious despair, blamed themselves for their child's $\mathrm{CF}$, disengaged from pursuing goals for their child, and consequently were more at risk for anxiety, depression, and emotional distress.

Growing evidence of depression among adolescents, young adults, and caregivers has emerged (Besier et al., 2011; Smith et al., 2010) as advancements in CF and prescribed intensification of daily treatments become part of daily care. Routine screening is currently done in some centers for adolescents and young adults with consideration to assessing the caregiver component.

While many studies depict a broad spectrum of experiences living with CF for both parents and specifically mothers, a conspicuous absence of studies that address experiences from a cultural or ethnic lens was evident. It was assumed the reviewed studies reflected the dominant Euro-American group and thus, underscored a gap toward 
illuminating cultural-ethnic views from mothers' stories of parenting a child with CF. In order to understand better the context of parenting and mothering a child with chronic illness from a Hispanic perspective, an overview of the Hispanic culture and family are discussed.

\section{The Hispanic Landscape}

A brief review of the changing diverse ethnic landscape is first offered to gain perspective about the significance of the panethnic context currently experienced in the U.S. The 2010 U.S. census (as cited in Ennis et al., 2011) reported the Hispanic population grew by $43 \%$, since 2000 , contributing to 4 times the total population growth of $10 \%$. Those of Mexican descent accounted for approximately three quarters of the increase. More than half the Hispanic population in the U.S. resides in three states; California accounted for $28 \%$. Individuals of Hispanic descent were found to have a median age of 27 years and are, therefore, younger than the major ethnic and racial groups (Pew Hispanic Center, 2013).

The Pew Hispanic Center (2013) is a nonpartisan research center that seeks to provide a greater understanding of the growing Hispanic population as it expands and affects the U.S. It is an offshoot of the Pew Research Center reporting trends, attitudes, and issues influencing the U.S. The report, Between Two Worlds: How Young Latinos Come of Age in America, presented findings from a nationally conducted survey, in both English and Spanish, from August through September 2009. The random sample was selected with an overrepresentation of 1,240 youths 16 years to 25 years of age. The Pew Hispanic Center detailed the magnitude of the most recent population growth and LatinAmerican dominated immigration wave: 40 million immigrants entered the U.S. since 
1965, of which about half were from Latin America. This number contrasts with the 14 million who landed in the U.S. during the European immigration wave of the 19 th century. Additional analysis from this report speculated the hardships and difficulty to progress were greater for the Hispanic group in contemporary society when contrasted with the upward mobility of the European immigration wave relative to four primary factors: (a) less need and use of skilled laborers with contemporary economic mobility, (b) illegal immigration status while trying to create a better life in the U.S., (c) fatalism as a worldview of the Hispanic culture is not consistent with values dominated by U.S. Anglo-Saxons, and (d) skin color as an additional barrier to assimilation where White Caucasians dominate. Placing the Hispanic group in a socioeconomic context enhances understanding of history and disparity.

\section{The Hispanic Family}

The family in the context of the Hispanic culture. Globally, family is cited as the dominant factor contributing to the well-being and welfare of children (H. I. McCubbin, Thompson, Thompson, McCubbin, \& Kaston, 1993; J. M. Patterson, McCubbin, \& Warrick, 1990; Viner et al., 2012). The family is the main institution that preserves and transmits culture and values, playing a key role in relationships and psychosocial development (Friedman, 1990; Lorenzo-Blanco, Unger, BaezcondeGarbanati, Ritt-Olson, \& Soto, 2012). These factors serve as a common thread that unite the fabric of both American and Hispanic families.

Familism, collectivism, and biculturalism. The emphasis on family, familism, among Hispanic people is a defining part of their ethnic identity, a distinction from the dominant Euro-American culture (Friedman, 1990; Lorenzo-Blanco et al., 2012; 
Quinones-Mayo \& Dempsey, 2005). Familism is defined as a strong identity and attachment among family members with their nuclear and extended family (SmithMorris, Morales-Campos, Castaneda Alvarez, \& Turner, 2013). Indeed, the strong ethnic values of familism might place additional tensions within the family as a result of the collectivism ideology versus the individualistic ideology of the Euro-American culture. Collectivism orients family members to place goals and interests of the group over those of the individual member (Fuligni, Tseng, \& Lam, 1999), whereas, the Euro-American orientation of individualism promotes the goals of each member toward independence and autonomy (Arnett, 2003). Collectivism represents aspects of family obligations that are a part of the Hispanic-Latino cultural values (Lorenzo-Blanco et al., 2012) and promotes family closeness and interdependence (respeto), trust among family members, and loyalty (familimiso).

Some studies (Arnett, 2003; Fuligni et al., 1999) lend support to a dynamic perception that cultural identity might vary by generations and by years following immigration within minority families that reside in the U.S. The notion that the first immigrated or the immigration-generation Mexican Americans might have a stronger orientation of collectivism than subsequent generations leads to an assumption of a bicultural posture and this stance might also incite tensions within the family (KossChioino \& Vargas, 1999). Although the Hispanic population is a heterogeneous group, the majority of individuals in the U.S. are from Mexico, Cuba, and Puerto Rico; the majority of Mexican American or those of Mexican descent reside in the Southwest, and thus are the dominant minority subcultural group (Ennis et al., 2011; Spector, 2004). While cultural and ethnic identities have commonalities, they are diverse among their 
distinct groups. This discussion is in no way intended to homogenize the uniqueness of Hispanics; however, for the purpose of this discussion, most information relates to the Mexican American cultural group, unless specified.

Generational issues, acculturation, and assimilation are important factors that influence family function and children's function within the family. Acculturation is the gradual acquisition of the dominant cultural elements for survival; it may be contrasted with assimilation where the process of learning the host culture's new values and behaviors is at the cost of losing their native culture (Friedman, 1990; Spector, 2004). It is helpful to distinguish these terms from ethnicity, which is often used synonymously with culture. Ethnicity gives a broader social distinction by including members of human groups from a common origin that might be differentiated by some elements but share behavior standards, cultural content, political, and economic considerations within a larger social system (Friedman, 1990; Koss-Chioino \& Vargas, 1999); Lorenzo-Blanco et al., 2012).

These social factors (acculturation, generations, and assimilation) intersect in a complex web with families, generations, gender, and ages and stages of development within a sociopolitical environment to construct individual meaning within their respective worlds. They lend understanding of demands upon already strained social situations. For example, there are concerns by some investigators that acculturation might contribute to the loss of the familism value (Elder, Broyles, Brennan, Zuniga de Nuncio, \& Nadar, 2005; Lorenzo-Blanco et al., 2012). There is also strong evidence that acculturation might impact the sociocultural adjustment of adolescents as a result of what has been called the widening "acculturation gap" (Juang \& Umana-Taylor, 2012, p. 4). 
This gap relates to situations in which adolescents and children gain values and behaviors of the host culture quicker than their parents, resulting in parental conflict. Socialization of Hispanic male and female roles has also been described as gender specific (Charmaraman \& Grossman, 2010; Domenech Rodriguez, Donovick, \& Crowley, 2009; Koss-Chioino \& Vargas, 1999; Lorenzo-Blanco et al., 2012) where females are more protected and interdependent in contrast to males who are granted freedoms and independence. Thus, the ability of Hispanic families to adjust to raising a child as newcomers in the U.S. thrusts the child, the parents, and thus the family, into a bicultural environment. Biculturalism describes individuals functioning between two cultures to fit the demands or circumstances of the moment. Biculturalism is defined as concurrently incorporating one's primary culture with the second or dominant culture (Friedman, 1990). Social changes reflecting bicultural identity and characteristics inherent in adolescence might add to parent and family tension and conflicts. For the adolescent who struggles with the additional issues of chronic illness, the burden of competing with family loyalty (familismo) might add dissonance to an already confounded situation in which the child or adolescent is compelled to conform as their normative focus shifts away from parents to peers, thus, creating a potential for familial tension.

Hispanic parents. Friedman et al. (2003) described Hispanic parent roles within traditional family stereotypes, noting they embodied an expected norm. For example, the masculine role was described as machismo featuring aggressiveness, superiority, insensitiveness, and invulnerability (Friedman et al., 2003; Lam, McHale, \& Updegraff, 2012; Varela et al., 2004). The father was described as subsuming an authoritarian role, demanding obedience and respect from the children, yet with little involvement in child 
care. The maternal role has typically been described as nurturing and self-sacrificing, defined by marriage and children (Friedman et al., 2003; Lam et al., 2012; Niska, 2001; Owen, Gonzalez, \& Esperat, 2013). While role stereotypes continue to perpetuate a monolithic view, recognition of more egalitarian relationships have become increasingly acknowledged (Friedman et al., 2003; Lam et al., 2012; Parra-Cordona, Cordova, Holtrop, Villarruel, \& Wieling, 2008; Niska, 2001; Vega, 1990).

Traditional maternal roles are also perpetuated throughout the literature (Friedman, et al., 2003; Gallagher, Gill, \& Reifsnider, 2008; Lam et al., 2012). One function of the maternal role was defined as a health promoter and protector for schoolaged child. Gallagher et al. (2008) unexpectedly found a fusion between healthpromotion and health-protector practices seeded in cultural themes of taking care and being mindful of their child's body, mind, and soul. Although the mothers were from lower socioeconomic levels, they adopted a holistic view, consistent with views of mothers from more educated and acculturated backgrounds. These findings underscore the definitive maternal role emphasized through parenting children in Hispanic families.

Hispanic nothers as caregivers. The topic of parenting from ethnic perspectives has gained greater interest recently. However, there are limited studies on parenting within the Hispanic sociocultural context in the U.S. (Grau, Azmitia, \& Quattlebaum, 2009; Hinojosa et al. 2012; Quintana \& Scull, 2009; Rehm, 2003). Cultural beliefs and values shape parenting approaches and are relevant, as they might color differences in how prescribed health treatments are carried out. Because of the paucity of studies specific of Hispanic mothers parenting a child with $\mathrm{CF}$, a review of studies reporting Hispanic mother's perspectives related to general parenting experiences and a review of 
limited studies focused on Hispanic mothers' experiences with chronic and special health care needs are discussed. The studies are primarily qualitative, limiting generalizability, and yet illuminate authentic narratives that represent voices of Hispanic mothers as caregivers.

Parenting studies were reviewed for both healthy children and for those with special health needs. The concept and practice of protective parenting was a recurrent theme within various contexts (Domenech Rodriguez et al., 2009; Gallagher et al. 2008; Koss-Chioino \& Vargas, 1999; Quinones-Mayo \& Dempsey, 2005; Rehm, 2000). Protective maternal parenting that was practiced with healthy preschool children focused on the holistic child through maternal observations, mindfulness, and vigilance (Gallagher et al., 2008). Rehm (2000) identified "keeping my child close to me" (p. 92) as a major dimension reported by Hispanic mothers' of children with a chronic health conditions. Quinones-Mayo \& Dempsey (2005) also described protectiveness as a hallmark of parenting their healthy adolescent females as they approached coming to age during their vulnerable adolescent years. Protectiveness as a parenting characteristic might be fundamental to the maternal role, particularly when a perceived threat is evident. Greater emphasis might be placed by Hispanic mothers relative to environments that are also foreign and outside their familiar Hispanic community.

Strom, Strom, and Beckert (2008) compared 739 maternal parents of 806 healthy adolescents, 10 to 14 years of age, from Hispanic, White, and Black groups with a previously established national standard to guide child rearing. The Parent Success Indicator was used to assess maternal behaviors related to the domains of Communication, Use of Time, Teaching, Frustration, Satisfaction, and Information 
Needs. Although findings suggested that across both generations, mothers' performance behaviors were rated favorably, Hispanic mothers rated themselves higher than mothers overall in Communication (e.g., good at listening, honesty, learning from friends, disciplining, discussing friends, accepting criticism), Teaching (e.g., teaching health, teaching care for others, teaching treating both genders as equals, teaching effort is key to success), Frustration (frustrated by following rules, peer influence, chores, manners, clothes, movies, music, phone use, study habits), and Satisfaction (e.g., likes child with family and friends, likes being with child, likes child's decisions, likes child's sense of responsibility, likes treatment from child, likes way with criticism). In contrast, Hispanic adolescents reported significantly lower scores for their mothers than was reported by adolescents overall. Both generations reported concerns about Use of Time while all mothers reported the need to learn more about what to expect of their child during teenage years. All three adolescent groups rated their mothers unfavorably in teaching them how to cope with stress.

Parra-Cordona et al. (2008) studied life experiences that most influenced the parenting of foreign- versus U.S.-born Latino parents. Commitment to parenting, their sense of responsibility, and satisfaction associated with their parent role were reported as similar. In addition, parents from both groups expressed contextual stressors that negatively influenced their parenting role, such as long work hours that diminished time from their children and fear of discrimination. Foreign-born Latinos reported more intense experiences of these contextual stressors. Language as a barrier was the major reported group difference. 
Hispanic nothers as caregivers of children with health conditions. Less is known about caring for a child with life-threatening illnesses within various cultural contexts. Hinojosa et al. (2012) examined White, African American, and Latino influences on the family resulting from serious chronic conditions. The highest percentage of married, two-parent households were from the Latino group. Other significant findings reported were: inability to travel, uncertainty if their child should be treated as special, living life day-to-day, and that no one understood the burden of their child's illness. No differences were found among the Latino groups (i.e., Mexican American, Puerto Rican, Cuban Americans, Central, and South Americans) after analysis by ethnic stratification controlled for sociocultural backgrounds and beliefs.

Hispanic mothers' experiences with parenting a child with respiratory problems were reviewed (Berg, Anderson, Tichacek, Tomizh, \& Rachelefsky, 2007; Robledo, Wilson, \& Gray, 1999). Two studies demonstrated consistent themes of fears and deficient or limited maternal knowledge (Berg et al., 2007; Robledo et al., 1999). These studies are also consistent with mother's health practices of febrile children where unsafe fever management was found because of a lack of mother's knowledge (Foronda \& Waite, 2008).

Rehm (1999) investigated the meaning of religious faith with Mexican American parents, primarily mothers, with a child with chronic health conditions. This study clarified that families used both religious faith and direction from the health care teams as support for health care decisions. It highlighted the manner in which Mexican American parents thread strong religious beliefs into the tapestry of their daily lives. This finding is 
in contrast to fatalism, as a passive process that attributes life's experiences in the hands of God, upheld by the dominant Euro-American culture as a common Hispanic belief. Maternal experiences with children with developmental delays (Reuda, Monzo, Shapiro, Gomez, \& Blacher, 2005) and raising a child with special needs (Hughes, ValleRiestra, \& Arguelles, 2008) were reviewed. Findings indicated that Latina mothers desired much the same for their children as most mothers, learning basic life and social adaptive skills, and communication with providers. Hughes's et al. findings represented voices from parents of children with special needs, most with cognitive delays, such as autism or learning disabilities. While the parents were aware their children required more time for care, they also desired for their child to be treated as normal, not disabled. Parents' goals were for their child to reach a level of independence. Normalization has been described as a common positive family adaptation in response to having a child with a chronic condition (Knafl \& Deatrick, 1986).

Three Brazilian publications underscored a somewhat different emotional tone than the U.S. literature. Tavares, Carvalho, \& Pelloso (2010) reported results of experiences of 16 mothers with children with $\mathrm{CF}$. Sorrow, doubt, suffering, and fear were themes reported in the abstract. The mothers noted that they had to reorganize their entire world and family. Pizzignacco, Mello, and Lima (2010, 2011) described CF's stigma and three family's experiences of CF. Descriptions reported from Tavares et al. and Pizzignacco et al. might demonstrate their connection to the strong cultural practice of familism, close familial relationships, and respective sociocultural norms. The language of Hispanic groups might emphasize emotion when translated to English. Language has been one of the strong factors singled out that perpetuates and preserves the cultural 
values of immigrated Hispanic groups (Friedman et al., 2003; Y. Padilla \&Villalobos, 2006). Language is also identified as a barrier with the Hispanic group related to health disparities. Health disparities as a social concern are reviewed next.

\section{Health Disparities}

While the Institute of Medicine's landmark report (Smedley et al., 2002a, b) increased consciousness about the nation's health disparities, it focused primarily on adults. More recent studies have outlined health disparities among children (Elster, Jarosik, VanGeest, \& Fleming, 2003; Flores \& The Committee on Pediatric Research, 2010). Flores and The Committee on Pediatric Research (2010) conducted an extensive literature review from 1950 through 2007. Findings for four major minority groups across health and health care spectrums highlighted persistent and pervasive health disparities when compared with White children. Although multiple methodological limitations were identified across several decades, these studies underscored health concerns for primary care, such as nutrition, activity, and general health behaviors, providing a snapshot of important and relevant factors. In addition, the report found that Latinos had a fair to poor overall health rating compared to Whites, including a higher incidence of tuberculosis and asthma. Multiple disparities for children with special health care needs, including higher rates of uninsured, no source of usual care, parent dissatisfaction with care, lack of subspecialist referrals, and unmet medical needs were described. These findings are consistent with the Institute of Medicine's report (Smedley et al., 2002b). Elster et al. (2003) reported similar results from a systematic review of racial and ethnic disparities for adolescents; these factors remain strong predictors of adolescent health care. Factors beyond immediate health issues included environment, neighborhoods, and support 
systems as well as health care system factors, such as access, utilization, and insurance. These various factors intersect to influence health care disparities that marginalize minority groups and remain an evolving area to increase understanding.

Speculation in understanding health care disparities range from multiple influencing dynamics that consider internal and external experiences of the health care system and those of patients and providers. Trust and confidentiality, fragmented systems, deficiencies of linguistics, materials, and a culturally diverse workforce represented some influences identified from health care systems. Contributing patient factors included health literacy that translated into misunderstood prescribed therapies and patient health and cultural beliefs that resulted in ineffective outcomes. Last, stereotypic beliefs from providers that might bias care approaches were thought to be additional contributors (Smedley et al., 2002a). When quality of care was used as a framework for health disparities, disparity findings remained consistent across both primary health care and chronic health conditions (Flores \& The Committee on Pediatric Research, 2010).

It is critical to understand experiences of parenting a child with $\mathrm{CF}$, within the sociocultural context, because of its marginalized position. It is also essential to discover meaningful information about Hispanic maternal perspectives on their health care experiences in order to deepen understanding that might lead to interventions to narrow disparities. Meleis and Im (1999) proposed that in order for nurses to advance knowledge of marginalized groups, awareness of history and the context of their marginalization must be understood. The study provided a space representative of Hispanic mothers' unheard perspectives, extended beyond structures and processes of health care systems 
and provider bias, and helped to uncover meaningful information toward more equitable care.

Health care experiences. Multiple barriers to a more equitable health care system exist. One approach to narrow the gap for marginalized groups is to increase appreciation of perspectives and experiences of their health care encounters. When families experience perceived equitable health care, satisfaction might be enhanced and thus, a narrowing of the health care gap might be realized. Communication has been linked as a quality indicator of health care encounters (Studer, Robinson, \& Cook, 2010). Communication can bridge relationship building that might facilitate meeting the needs of marginalized groups that report feeling misunderstood and dissatisfied with health care encounters. Although existing health disparities have been established, less is known about experiences of families with children who have special health care needs or of Mexican American and Hispanic minority families. Dissatisfaction with health care services, the cumbersome navigations of health care services, linguistics used during interviews (Ngui \& Flores, 2006; Perry \& Ireys, 2001), and less satisfaction with the primary care providers versus the specialty care providers for children with special health care needs were found as contributing factors (Perry \& Ireys, 2001). Likewise, Hispanic families experienced less overall participatory decision making compared to non-Hispanic parents (Xu, Borders, \& Arif, 2004). Negative attitudes toward health care services, low levels of satisfaction with care, low confidence in care providers, as well as feelings of being misunderstood were also reported from Hispanic mothers' health care experiences (Clark \& Redman, 2007. 
In Hispanic families, women's roles have largely been assigned as taking care of children, the child's health, and making health care decisions (Clark \& Redman, 2007; Sanchez-Birkhead, Kennedy, Callister, \& Miyamoto, 2011). Social support is integral to the Hispanic culture and related to significant health outcomes (Y. Padilla \& Villalobos, 2006). Immigrant Hispanic women's process of health care decision making is predominately through a social network and advice from family members and friends (Sanchez-Birkhead et al., 2011). These researchers described immigrant women's health care practices and behaviors as negatively affected by isolation and lack of social support as a result of immigration. These findings have relevance for Hispanic families with a child with $\mathrm{CF}$ in relation to infection control practices where avoidance of others with $\mathrm{CF}$ is recommended to protect from potential transmissible pathogens. Infection control practices might magnify added feelings of isolation within the Hispanic community and merit further investigation. In addition, feelings of isolation might stem from having a child with a condition dominant in the Euro-American population and thus, in the CF community.

\section{Hispanic Mothers' Health Behiefs}

It is well documented that Hispanic, specifically Mexican Americans, ascribe to health beliefs originating from the ancient Hippocrates theory of four body humors (Friedman et al., 2003; Owen et al., 2013; Spector, 2004). Body humors comprised elements of hot or cold and wet or dry as the basis of many health beliefs. A holistic view of life is embraced in which health is viewed as a balance between the elements and the natural and supernatural world (Friedman et al., 2003). Preventive beliefs and rituals are executed to maintain this balance through prayers, faith, herbs, and folk rituals. Illness is 
viewed as an imbalance of the elements. It might also be viewed from folklore stance, for example, the evil eye (mal de ojo) viewed as illness caused by envy or the view that illness was caused from fright or emotional trauma (mal de susto). In addition, studies have also underscored that the cultural folk practices and health beliefs are not mutually exclusive and do coexist, blending both Western practice with cultural beliefs (Owen et al., 2013; Y. Padilla \& Villalobos, 2007). While health beliefs rooted in cultural heritage are commonly recognized, less is known about their role in Hispanic families of children with CF.

Cultural health beliefs and attitudes shape circumstances' meaning for which a family seeks medical attention. It is prudent for health care providers to be sensitive to cultural differences, stereotypes, and origins of cultural beliefs and practices. Exploring the meaning parents ascribe or the views they have regarding their child with a chronic condition, from within their family and broader sociocultural context, lends important information to tailor treatments. Questions specifically as to what the family believes caused the condition, the expected prognosis, and the child's anticipated social role also give insight into cultural beliefs and practices that might diverge from the dominant Euro-American views (Groce \& Zola, 1993). As noted earlier, mothers' roles in rearing and caring for the health of their children are expected. Therefore, also understanding women's health beliefs and practices might shed insight into influences exerted on their children.

Sanchez-Birkhead et al. (2011) described the social network Hispanic women rely upon for their health decisions. Other areas highlighted outlined the combined traditional use of home or alternative therapies with Western therapies, consistent with other studies 
(Y. Padilla \& Villalobos, 2007). An unexpected finding reported perceptions of discrimination where the individuals felt marginalized because of language and physical appearance (ethnicity), and thus, impeded the ability to establish trust with the provider as a result of limited time as well as these aforementioned factors. Hispanic women's health care experiences can influence expectations and experiences of parenting their child with a chronic condition. Y. Padilla and Villalobos described the collectivism view and its relationship with familism that connect interdependently to take care of the elderly within the Hispanic family, regardless of the perceived burden. It then follows that parents would take care of their chronically ill child regardless of the perceived burden.

The significance of the limited studies highlights the potentially often covert practices of home or folk remedies that might not be shared with the health care providers (Bearison, Minian, \& Granowetter, 2002; Foronda \& Waite, 2008). Gaining an understanding of cultural health beliefs and practices might provide insight into the family's approach to care for their child with $\mathrm{CF}$ and carrying out prescribed therapies.

Acculturation. Several studies reviewed in the discussion considered factors of acculturation. Acculturation, as noted, is the adoption of customs, beliefs, and values of the dominant society (Friedman, 1990; Koss-Chioino \& Vargas, 1999). Acculturation is a complex concept that has included models and theories extended throughout the past century to encompass more than contact between a new and host group. More recent models of acculturation have considered an individual's choice to acculturate versus the group. Of the reviewed studies, a notable finding for less acculturated parents included higher reports of involvement with physicians in participatory decision making (Xu et al., 2004). Explanations offered related to lower expectations for the physician visit, or 
expectations that were met, or the physician made a greater effort because of the language or social disparity. In addition, less acculturated mothers reported less satisfaction with the lack of perceived personal attention from the health care providers' to the mothers when seeking care for their child; wanting to feel a stronger connection with their health care provider, perhaps trust was an implied concern (Bearison et al., 2002). Two studies highlighted similarities between less acculturated and more acculturated mothers in regard to maternal roles. Gallagher et al. (2008) found less acculturated mothers shared parallel holistic views of their child and health promotion with those of more acculturated mothers. Findings from Clark and Redman (2007) suggested that the expectations of less acculturated mothers for fast access to health care and to find a diagnosis and treatment for their ill child were similar to expectations of more acculturated mothers. Sanchez-Birkhead et al. (2011) reported that less acculturated women felt discrimination, were affected by the loss of their familiar strong women-towomen support, and felt isolated because of their change in residence and living arrangements, thus, affecting seeking medical assistance.

In their review of their prior work, A. M. Padilla and Perez (2003) discussed a decline of cultural awareness from first to fourth generation of Mexican origin respondents, with the steepest decline between first and second generations. However, the finding of ethnic loyalty up to the fourth generation remained consistently high. These findings indicated that while Mexican individuals possessed little information of their cultural heritage, they continued to identify with their ethnic roots, particularly related to social factors, such as selection of friends. Furthermore, these authors discussed 
discrimination as a strong factor in ethnic identity. Indirect exposure or beliefs of discrimination were sufficient to fuel stronger ethnic identity.

Numerous measures were used to address acculturation among the reviewed studies. An accurate measure of acculturation has been difficult because of its complexity. Studies have used various definitions of acculturation as well as measurement tools, leading to inconsistent findings as a result of its multidimensional nature (Thomson \& Hoffman-Goetz, 2009; Wallace, Pomery, Latimer, Martinez, \& Salovey, 2010). While acculturation is an important dimension to evaluate, its measurement presents ongoing challenges.

\section{Summary of Gaps in the Literature}

$\mathrm{CF}$ is a rare, complex, high-burden, high-cost chronic condition occurring most frequently in the non-Hispanic Caucasian population. With the Hispanic population on the increase in the U.S., the incidence of $\mathrm{CF}$ in this group most likely will increase. There is mounting evidence that Hispanic individuals with $\mathrm{CF}$ have a worse life course trajectory. Scant literature specific to $\mathrm{CF}$ and the Hispanic population residing in the U.S. addresses their unique needs, reinforcing them as a marginalized group within the greater $\mathrm{CF}$ community. The Hispanic people bring a rich heritage that contributes to the diverse landscape in the U.S. Their voices have not been heard amidst those of the dominant Euro-American group in the $\mathrm{CF}$ community. Through peeling back cultural layers, a space is opened for stories to be told and to gain perspective of their sociocultural realities with $\mathrm{CF}$. CF specific to the Hispanic-Latino cultural group was reported in three Brazilian publications (Pizzignacco et al., 2010, 2011); Tavares et al., 2010) providing a different lens for experiencing the world of $\mathrm{CF}$, inclusive of some similarities and 
differences. A plethora of CF studies exist that have illuminated living with $\mathrm{CF}$ and advanced scientific discoveries; however, studies in the U.S. specific to Hispanic individuals mask any unique findings as a result of underrepresentation. The existing gap in the literature underscores the importance of addressing this pressing issue to gain perspective from personal stories of mothers raising a child with CF. Descriptions of maternal experiences, as well as perspectives of health beliefs, and perceptions of health care experiences related to $\mathrm{CF}$ have paved the groundwork for development of future culturally sensitive intervention studies. It is past time to improve the understanding of their strengths, concerns, and vulnerabilities. 


\section{Chapter 3: Method}

\section{Statement of Purpose}

This chapter discusses the qualitative methods applied to the study. The sample, data collection, and analysis of data methods are presented. The study's protection of human subjects and trustworthiness are discussed.

This study's purpose was to explore and describe experiences of mothers parenting a child with $\mathrm{CF}$ from a Hispanic perspective in order to increase understanding from their sociocultural lens. In addition, this study proposed to gain insight as a first step to develop ultimately more culturally congruent interventions to improve patient outcomes. The study sought to uncover and describe health beliefs and health care experiences of Hispanic mothers relative to the care of their child with CF. The study's specific research question asked: What is it like for you as a Hispanic mother to parent your child with CF? A second research question aimed to gain an understanding of maternal health beliefs and health care experiences related to their child's CF.

\section{Specific Aims of the Study}

The specific aims of the study were to:

- Aim 1: Explore and describe Hispanic maternal perspectives and experiences of parenting a child with $\mathrm{CF}$.

- Aim 2: Explore and describe the health beliefs from Hispanic maternal perspectives related to health care of their child with $\mathrm{CF}$. 
- Aim 3: Explore and describe health care experiences from Hispanic maternal perspectives related to care of their child with $\mathrm{CF}$.

\section{Qualitative Research}

Qualitative tradition is appropriate to study phenomena not yet fully described or understood. A goal of qualitative research is to gain a holistic view of the context under study (Miles \& Huberman, 1994; Polit \& Beck, 2012). A main task is to elucidate the ways people come to understand, describe, and take action to manage their daily situations (Miles \& Huberman, 1994). According to these scholars, qualitative research generates strength from occurring in the natural setting arising from ordinary events reflective of real life events. In addition, it is particularly well suited for exploring narratives not yet told. Flexibility is a hallmark of qualitative designs where the researcher becomes the research instrument and procedures evolve to capitalize on findings that emerge during the time of the study (Polit \& Beck, 2012). A qualitative design was a good fit for this study because the phenomena of interest required a defined foundation to integrate understanding through exploration and descriptions of Hispanic mothers' perspectives and experiences. It brought forth the real-life situation of their present experience as mothers. This approach lent itself to family studies in which the quest was to understand the holistic perspective of the maternal experiences within their CF world.

Qualitative research methods encompass many designs rooted from multiple traditions and disciplines. Narrative inquiry is one such approach that has gained renewed interest across multiple disciplines in recent years because of its pure nature of storytelling, as stories are lived and told (Riessman, 2008; Sandelowski, 1991). Personal 
narratives are the stories told about personal experiences; they are part of the study of everyday life and everyday life performance (Langellier, 1989). Clandinin and Connelly (2000) provided major contributions to narrative research or inquiry, from a scholarly, narrative, academic lens. The authors stated, "Context makes all the difference" (p. 26). The discussion that follows clarifies the use of terms between story and narrative, in the context of narrative inquiry and the current study.

Narrative. Narrative has a historical trail of debate across disciplines and time. For example, debate exists related to definitions, historical origin, and its growth as a medium used in social science and human research. The term narrative historically has been associated with literary works, but more recently has been applied to social and human sciences (Riessman, 1993). The "narrative turn" (Wells, 2011, p. 9) in the latter part of the $20^{\text {th }}$ century (also a source of debate), meaning the point in time where narrative gained greater attention and application in research, has been embraced across diverse disciplines such as anthropology, psychology, sociology, education, nursing, and medicine (Chase, 2005; Riessman, 1993). Narrative, simply put, means "a story that is told or written" (Merriam-Webster, n.d. Multiple scholars have described narrative with a caveat for its numerous associated meanings and uses crossing boundaries of various disciplines (Chase, 2005; Clandinin \& Connelly, 2000; Langellier, 1989; Riessman, 1993, 2008). Two noteworthy definitions of narrative Riessman (2008) cited are: first, as oral storytelling, events are told connecting sequences that are subject to later action and that focus on the meanings the teller wants the listeners to take away; second, events perceived as important by the speaker are selected, organized, connected, and evaluated as having meaning for a select audience. Polkinghorne (1988), from the lens of narrative 
in psychology, referred to narrative as making of the story, the cognitive structure of a story, or the result of the process, such as stories or tales.

Narrative and story frequently are used synonymously, lacking a uniform definition, although distinctions have been described (Paley \& Eva, 2005; Riessman, 2008). One distinction of narrative is that it lends order and meaning to events that might be told in an otherwise random and disconnected sequence (Riessman, 2008). The personal narrative is the Labovian model characterized by six structural elements. This model referenced degrees of narrative completeness (Langellier, 1989). Riessman addressed narrative on a continuum: one end having an extended discourse of discrete units in response to a single question from an interviewer, rooted from a social linguist lens. Anchored on the opposite end, narrative is life story (i.e., biography), based from anthropology and social history. Situated in the middle of the continuum is the personal narrative influenced from psychology and sociology. Riessman's perspective parallels Paley and Eva (2005) where these authors distinguished narrative from story as a hierarchy. They described narrative to contain texts as having a high or low degree of "narrativity" or "something that text has degrees of, borrowed from a literary concept" (p. 85). Applying the concept of narrativity, story was ranked with a high degree, characterized with an interweaving plot and character, constructed to provoke an emotional response by how it is organized. Narrative had a low degree ranking of narrativity, portrayed as sequenced events and causal connections linked between them. A staircase illustrates this concept metaphorically with narrative located at the lowest step and story at the highest step (Figure 1). Thus, this metaphor depicts the point that all stories are narratives, but not all narratives are stories (p. 85). Their final analysis 
recommended researchers to maintain a distinction between these terms. Thus, the following Paley and Eva's definitions are adopted for the purpose of this study: narrative is the ordering at least of one or more events causally connected; story refers to the threads of plot and character woven together the structure of which is designed to provoke an emotional response from the audience or reader. Narrative, stories, and personal stories are all used in narrative inquiry, discussed next.

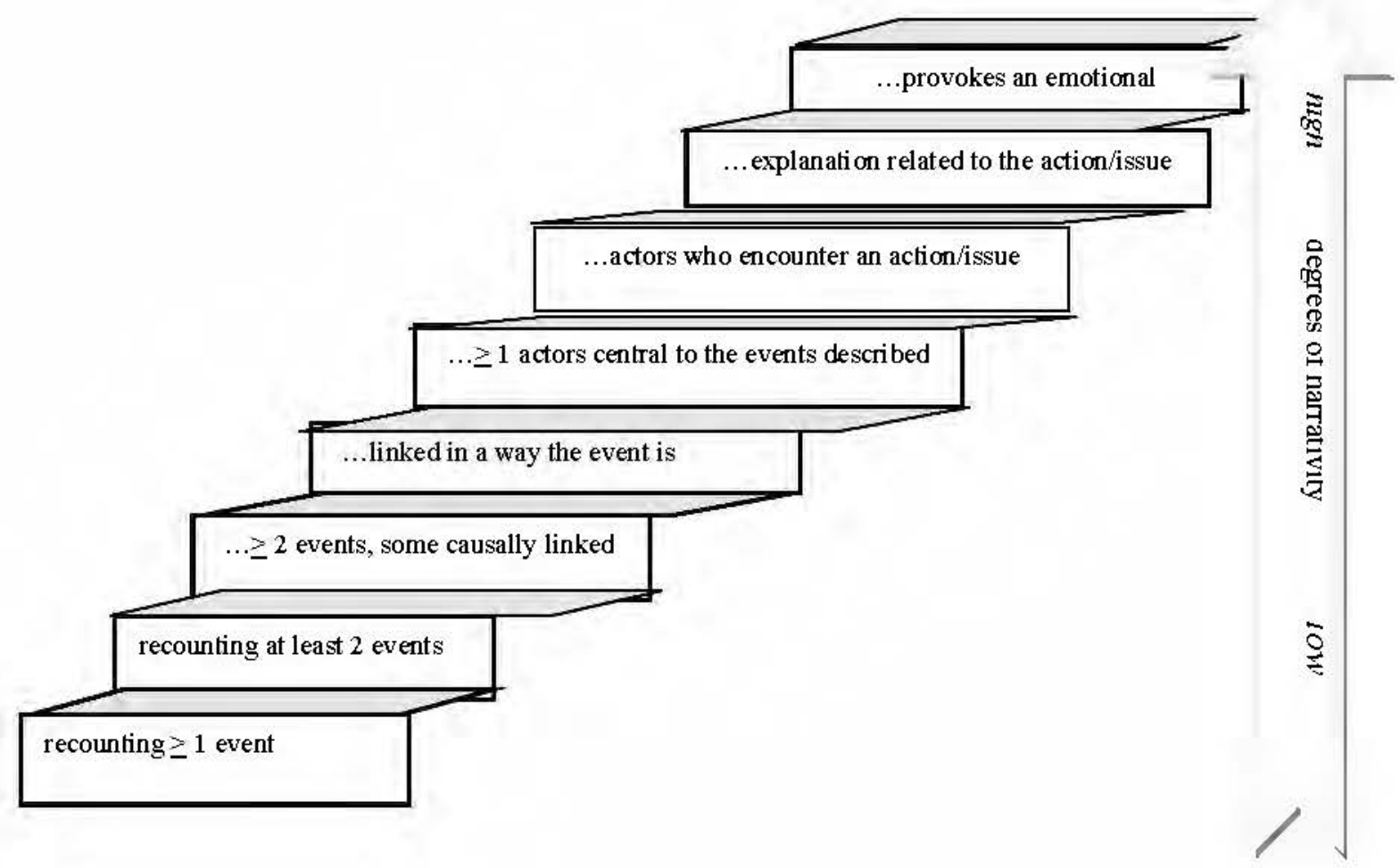

Figure 1. Adapted from "Narrative Vigilance: The Analysis of Stories in Health Care by J. Palely and G. Eva, 2005, Nursing Philosophy, 6, p. 87, (C) Blackwell Publishing. John WileyC 2005.

Narrative inquiry. Narrative inquiry, defined by Schwandt (2007), is "encompassing the interdisciplinary study of activities involved in generating and analyzing stories of life experiences... and reporting that kind of research" (p. 203). 
Examples include life histories, narrative interview, personal stories, journals, or diaries. Although storytelling has existed for a long time, narrative inquiry, as in qualitative research, is both a method and a process. Dating back to the early $1960 \mathrm{~s}$, social scientist Labov recognized that events from the past that are retold represent significant aspects of individuals' social realities (as cited in Holloway \& Wheeler, 2010). This approach provides a reflective space for mothers to make meaning of their experiences. Although there have been concerns about a story's accuracy or possible alteration as a result of the teller's selective memory around its reconstruction, enriched meaning is found in the retelling of events that become molded into significance. Holloway and Wheeler described narrative inquiry as a way to disclose intentions and motives of human beings to the researcher.

Clandinin and Connelly (2000) suggested narrative inquiry begins with life experiences retold in stories, where "living, telling, retelling, and reliving etch the qualities of a life" (p. 187) These scholars further described narrative inquiry as the study of experience, where experience primarily relates to people situated temporally within their contextual world. Its purpose is to provide opportunities to tell and retell, thereby reconstructing the storied experience into new possibilities, new directions, and new ways of doing from the reconstructed and relived insights. Thus, it is reflective of knowing that it is a never-ending story as it is retold in time and unfolds growth with time. Riessman (2008) highlighted this concept, where the investigator coconstructs the narrative with the mother, clarifying discourse for accurate representation. The relationship in the coconstruction of narrative is key to the narrative inquirers' role.

Natural inquiry principles guide narrative inquiry to describe and understand the 
phenomena of interest not yet uncovered from ordinary everyday experiences (Marshall \& Rossman, 2011). Storytelling opens a space to allow the emergence of rich description oriented in its natural setting, not manipulated or reduced to statistical generalizations.

Inquiry through stories from Hispanic mothers who have a child with $\mathrm{CF}$, offered an understanding of their day-to-day experiences and perspectives. It captured a deeper knowing about parenting a child with $\mathrm{CF}$ from the nondominant lens within the $\mathrm{CF}$ community. Storytelling was explicitly selected to create a space for Hispanic mothers to share personal stories of their perceptions and experiences of parenting their child with $\mathrm{CF}$, as actors in their own world, described in their own words. Thus, mothers' views provided clarification that add to family studies and situate families that have a child with $\mathrm{CF}$ in their unique world, as it exists. Overcash (2004) described narrative inquiry as a research method that links well with understanding the effects a disease and/or illness might have on patients or their significant others or both. In addition, personal stories represent experiences, both ordinary and significant events, in one's life (Chase, 2005). One might suggest that parenting a child with a genetically transmitted, life-shortening, chronic condition is a highly significant life event. Thus, the aims of this study are consistent with narrative inquiry within the qualitative tradition. This study's findings have added to knowledge of this underrepresented ethnic group and will inform ultimately a larger multicenter study of U.S. Hispanic families with CF. It extended understanding factors beyond genetics related to this group, elucidated their health beliefs and experiences, and enlarged and deepened the understanding of relationships within their sociocultural constructed realities.

Sensitized frameworks. Critical theory and feminist theory constituted the 
study's philosophical underpinnings. Although each paradigm belongs to a larger family of philosophical approaches and strategies, they share significant elements (Campbell \& Bunting, 1991). Specifically, they both share a focus on the centrality of power relations in social processes, including individual's experiences within the health care system. Rooted in these philosophical underpinnings, the expectation that the researcher is devoid of preconceived thoughts, experiences, or observations was questioned. Qualitative researchers within the critical-feminist paradigm assert that all research has shades of political elements derived from multicultural, gendered, interpretative arrangements, thus challenging the traditional assumption of neutrality (Marshall \& Rossman, 2011). Narrative analysis as a critical genre departs from the qualitative tradition in that it seeks to describe the meaning of experiences of those who are frequently marginalized or oppressed. Personal narratives are foundational to understanding underrepresented, and often, muted lives. It assumes that all people build narratives as a process of construction, deconstruction, and (re)construction of their life tales, which is a central belief to this approach (Marshall \& Rossman, 2011).

Critical theory originated in the 1950 s in the Frankfurt School, associated with the Institute for Social Research at the University of Frankfurt am Main. It emerged about the same time as the second feminist wave in the U.S. (Campbell \& Bunting, 1991; Mohammed, 2006). Critical theory does not have a unified definition but attends to power relations and underlying societal forces that result in inequalities. Both the critical and the feminist paradigms share the following: (a) knowledge is socially constructed and does not exist outside the context from where it was constructed; (b) understanding patterns of human behavior involves understanding both personal meanings of social 
structures and the meanings of those structures from the greater community; and (c) structures might have resulted in class oppression, with feminism more focused on the fundamentals of gender oppression (Campbell \& Bunting, 1991).

In this study, stories told by Hispanic mothers interacting with the health care system revealed untold, yet important, perspectives to heighten awareness and develop empowerment strategies to reduce disparity concerning power relations in routine health care encounters. Lanser (1986) described the difference between private and public narrative levels relevant to women's text, which was related more toward literary writers. However, she pointed out that women writers traditionally wrote for private audiences rather than the public patriarchal audiences. The dichotomy of private and public with female and male is a complex intersection that might have ethnic influence undertones of traditional gender roles within a changing cultural context. Likewise, both paradigms embrace historical and contextual influences as important information elements from subjective perceptions and experiences as knowledge. Feminism, in contrast to critical theory, envisions feelings that are respected, valued, and viewed as having equality and emancipatory potential (Campbell \& Bunting, 1991). Thus, the mission for both critical theory and feminist theory is creating and using knowledge for emancipation.

\section{Study Setting}

The study sample was accessed through an accredited CFF center, where children with $\mathrm{CF}$ receive specialty health care services. The $\mathrm{CF}$ center was located in a large metropolitan children's hospital in the Southwest U.S. This study site was selected because it is an established CF care center in an ethnically diverse urban area. The 
interview location was determined individually with each mother and was scheduled based on her preference.

\section{Identification and Selection of the Study's Participants}

Purposive convenience sampling was used to yield the richest responses. After an Institutional Review Board (IRB) reviewed waiver (Appendix A) for use and disclosure of protected health information, access to the CFF Patient Registry database was obtained. The database was searched for eligible Hispanic families. Demographics in the database include self-assigned ethnicity, identified by parents, and entered by trained hospital staff through a secure database. Inclusion criteria included biologic mothers who self-identified as Hispanic ethnicity and who had a child between the ages of 1 to 21 years diagnosed with $\mathrm{CF}$ for at least 1 year, The child with $\mathrm{CF}$ might have known $\mathrm{CF}$ associated complications, such as CF-related diabetes or chronic sinusitis; but excluded for coexisting diagnoses, such as autism, ADHD, or developmental delays. Mothers were English or Spanish speaking. A Spanish interpreter was offered and provided when mothers indicated a preference.

Of 140 registry CF patients, a list was created of 43 eligible Hispanic patients who receive health care from the study site. The list was reviewed with the CF social worker to identify known Spanish-speaking mothers. The CF clinic list was reviewed weekly to identify any scheduled potential families in order to introduce myself and the study information to interested candidates. In addition, once language preference was clarified, an interpreter used a script (Appendix B) when contacting potential candidates by phone and for follow-up contact to schedule the interviews. I realized the list of 43 eligible patients did not equal 43 eligible mothers because some families had more than 
one child with CF. Four eligible mothers had a combined total of thirteen children listed -34 eligible mothers remained. Eligible candidates were further reduced by exclusion criteria. Two patients identified as Hispanic ethnicity did not have Hispanic mothers; two patients had nonbiologic maternal caregivers; two children were less than 1 year after diagnosis; one child had medical complications beyond $\mathrm{CF}$; and one father was the primary caregiver. Twenty six mothers remained as eligible candidates; two mothers declined to participate: one because of inconvenient timing; one stated she felt she would not contribute anything because her family was too Westernized. Twenty four eligible mothers remained. One interview was excluded when the mother stated during the interview she was unsure if her daughter's CF diagnosis was being reclassified. A CF physician confirmed the diagnosis was reclassified to CF-related metabolic syndrome, thus, she was excluded. The sample size was reached when no new data significant to the study were forthcoming (Holloway \& Wheeler, 2010) - otherwise described as reaching data saturation. The final number of maternal interviews was 10 .

Recruitment was initiated following review from the IRB. Eligible candidates were entered on a list (Appendix C) created from the CFF Patient Registry, subsequent to an IRB-reviewed waiver, and were contacted, provided information about the study, and invited to participate. An IRB-reviewed announcement in English and Spanish was posted in the quarterly CF newsletter (Appendix D), distributed by both traditional mail and e-mail, inviting Hispanic mothers to contact the investigator for further information to participate in the study. Eligible mothers were approached at a routine outpatient visit by myself or contacted by phone. An IRB-reviewed information sheet in English and Spanish describing the study and participation expectations were offered to eligible 
mothers by me or through the interpreter as indicated (Appendix E). The study was explained; candidate agreement or declination was confirmed. Volunteered mothers were enrolled and additional information was entered on the log. An interview date or agreement to call at a later time to schedule the interview was verified. Mothers were contacted by phone the day prior to the interview to confirm date, time, address, and available parking. A reimbursement check for $\$ 20.00$ was sent toward transportation expenses after final member checking was completed. The majority of mothers were recruited by personal contact and explanation of the study during their routine CF clinic. One mother responded from the CF newsletter announcement.

\section{Human Subject and Ethics Considerations}

The IRB reviewed the study and protocol at the participating hospital study site and the University of San Diego, Hahn School of Nursing and Health Science reviewed them prior to mothers' enrollment. Explanation of the study and purpose were provided. In addition, risks and benefits were reviewed, as well as the voluntary nature of participation and study confidentiality. After clarifying questions, all participants signed informed consents in a face-to face encounter in either English or Spanish prior to the interview (Appendix F, G) with other IRB forms completed (Appendix H, I). A copy was offered to the mothers and mailed to them at their request. All participants' data were coded and stored in a password protected computer. Informed consents, completed interviews, field notes, and digital audio tapes were stored in a locked file drawer or password protected computer in my office. All data, except for the signed consents, were coded with a unique identification number and assigned a pseudo-name to protect confidentiality. The mothers were reassured of voluntary participation and that they could 
stop the interview or withdraw from the study at any time. This information was again emphasized with member checking and subsequent contact to clarify interview information. Confidentiality, anonymity, and reassurance that the medical care, management, or treatment of their child would not be affected by declining or participating in the study were emphasized.

Study risks were explained, which included potential emotional stress when discussing their child's CF in the context of its life-shortening nature. It was also considered that mothers might find discussion of the study topic helpful, particularly if such opportunities had not been addressed in the past. The therapeutic benefit of personal narratives and those related to health and illness has been documented (Frank, 1997; Hunt, 2000). Cystic fibrosis team physicians are on call 24 hours a day, seven days a week and were able to assess emotional concerns had this occurred. In addition, information provided in the informed consent, connecting mothers to resources for support, if indicated, through community mental health services was reviewed.

\section{Data Collection Procedures}

Interviews were conducted over a 5-month period. Seven interviews were conducted in mothers' homes, according to their preference. Three mothers requested to meet at the hospital, where a private office was secured to provide confidentiality. The initial interview averaged about 1 to $1 \frac{1}{2}$ hours. A second phone interview was conducted with two participants to clarify information, which averaged about 15 minutes. One unsuccessful attempt was made to clarify additional information. Voluntary participation, confidentially, and audio recording were emphasized.

Demographic information was collected prior to the interviews (Appendix J). This 
information was digitally audio recorded following the second interview to capture salient details. I realized important information emerged when talking about demographics that would have been lost using paper and pencil format alone. All interviews were digitally audio recorded in a face-to-face interview consisting of semistructured open-ended questions. The interview began with introductions and stated the study's area of interest, followed with an open-ended request addressing Aim 1: Tell me your story, as a Hispanic mother, of your experience of what it is like to take care of your child with CF. Although flexibility in the interview followed the mother's conversation path, semistructured questions were used to gain meaningful information that addressed the study aims (see Table 1).

\section{Table 1}

Semistructured Interview Questions

\begin{tabular}{ll}
\hline \multicolumn{1}{c}{ Study Aims } & \multicolumn{1}{c}{ Questions } \\
\hline Aim 1: Explore and & 1. Tell me your story of what your life is like being a parent \\
describe Hispanic & to your child with CF; (a) What makes it easier?; (b) What \\
maternal perspectives & makes it harder?; (c) What part of being a parent to your \\
and experiences of & child with CF is different or the same as being a parent to \\
parenting a child with & $\begin{array}{l}\text { your other children (or extended relatives, for an only } \\
\text { CF. }\end{array}$ \\
& child?); (d) Tell me what does your child having CF mean to \\
& $\begin{array}{l}\text { you?; (e) What is it like to think about (child's name) future? } \\
\text { Hispanic mother, experience as having influence on }\end{array}$ \\
& parenting your child with CF.
\end{tabular}


3. Tell me what gets you through each day.

(Table continues)

\begin{tabular}{ll}
\hline \multicolumn{1}{c}{ Study Aims } & \multicolumn{1}{c}{ Questions } \\
\hline Aim 2: Explore and & 1. What do you understand to be the cause of [child's name] \\
describe the health & CF? \\
beliefs from Hispanic & 2. What do you understand how to treat it or what cures it? \\
maternal perspectives & 3. Some parents have told us they have tried remedies from \\
related to health care of & their "home country"; What have you tried (Carteret, 2011)? \\
their child with CF. & 1. What has your experience been with the health care for \\
$\begin{array}{l}\text { Aim 3: Explore and } \\
\text { describe health care }\end{array}$ & CF that [child's name] receives? \\
experiences from & 2.Can you describe a situation where you felt support \\
Hispanic maternal & related to the health care of your child? \\
perspectives related to & 3. Regarding your child's health care, some Hispanic \\
the care of their child & parents tell us they have felt disrespected or experienced \\
with CF. & been with your (child's name) CF health care (Sanchez- \\
\hline
\end{tabular}

Additional questions related to Aim 1 from the interview schedule included: (a) What makes it easier?; (b) What makes it harder?; (c) what part of being a parent to your child with CF is different or the same as being a parent to your other children (or your extended relatives for an only child)?; (d) Tell me what does your child having CF mean to you (Zoucha, 1998)?; (e) What is it like to think about (child's name) future with 
having CF? Tell me about the issues or concerns you, as a Hispanic mother, experience as having an influence on parenting your child with CF. Tell me what gets you through each day.

Aim 2: Explore and describe the health beliefs from your Hispanic maternal perspectives related to health care of your child with $\mathrm{CF}$.

A semistructured approach was considered to be most useful to represent the voices of Hispanic mothers with $\mathrm{CF}$ about their health beliefs. Semistructured questions included the following:

1. What do you understand to be the cause of [child's name] CF?;

2. What do you understand how to treat it or what cures it?; and

3. Some parents tell us they have tried remedies from their "home country"; what have you tried (Carteret, 2011)?

Probes were posed based on mothers' responses.

Aim 3: Explore and describe health care experiences from your Hispanic maternal perspectives related to the care of your child with $\mathrm{CF}$.

Aim three included the following:

1. What has your experience been with the health care for CF that [child's name] receives?

2. Can you describe a situation where you felt support related to the health care of your child (Xu et al., 2004)?regarding [child's name] CF and his or her health care?; and

3. Some Hispanic parents tell us they have felt disrespected or experienced discrimination, I am wondering what your experience has been with your 
child's CF care (Sanchez-Birkhead et al., 2011).

Probes, such as: Can you tell me more about that?, recalling a prior statement or a request to talk further about a statement were introduced based on the maternal responses.

Wrap-up questions were introduced to facilitate closure of the interview, such as: You have provided much important information, is there anything else you want to tell me about what it is like being a parent and taking care of [child's name] with $\mathrm{CF}$, and/or what would you like me to remember and write about most from our talk today?

Field notes were recorded during and after the interviews. Notations on environment surroundings, maternal affect, comfort level of interview process, and nonverbal gestures or comments that stood out were made. After the interviews, I reflected on responses that stood out and noted associations or questions that called my attention, recorded as memos in field notes. In addition to taping the demographic questions, modifications to probes were added. For example, the word disrespect was added by asking if the mother had experienced any disrespect or discrimination with her child's health care to augment the range of potential experiences and cultural sensitivity (respeto). All interviews were digitally audio taped and transcribed verbatim using Dragon Naturally Speaking, version 12 by myself. A bilingual Spanish-speaking interpreter was present at interviews when mothers indicated a preference. In these interviews, translation was simultaneous, which facilitated transcription. An iterative process informed ongoing data collection refinement.

Demographic data included primary caregiver of the child with $\mathrm{CF}$, maternal and paternal ages, mother's highest completed education level and whether mother worked outside the home, primary language spoken in the home, self-identified ethnicity and 
country of origin, and insurance type as a proxy for socioeconomic status. Additional information collected was number of household members and their familial relationship; number, ages, and gender of children in the household, identifying those with CF; recall of participation in genetic counseling; and household members who smoke tobacco. Participation in genetic counseling is a standard practice after a confirmed CF diagnosis. Participation in genetic counseling, or a lack of, might suggest an unexplored dimension of health disparities. Language spoken in the home has been described as a predictor of ethnic identity and acculturation (Friedman, 1990). Air pollutants, including passive exposure to tobacco, have been shown to have deleterious effects in individuals with $\mathrm{CF}$ and might play a role in health disparities (Wolfenden \& Schechter, 2009). Data from the electronic medical record included the most recent results of forced expiratory volume percent predicted in 1 second $\left(\mathrm{FEV}_{1}\right)$. The child's age, age at diagnosis, documentation of CF by sweat test, or/and CF DNA analysis were verified from the CFF Patient Registry. The severity of lung disease inherent in CF might influence the experience of parenting. Therefore, $\mathrm{FEV}_{1}$ percent predicted, a common measure of lung function, was obtained to describe the child's pulmonary severity (Fiel, FitzSimmons, \& Schidlow, 1994). Data were extracted from the $\mathrm{CF}$ outpatient electronic medical record within 1 week following data collection. Demographic information helps to locate mothers' stories within their social cultural landscape.

Five mothers requested a Spanish interpreter. All of the five expressed they understood some English, but Spanish was their primary language. Because the study focused on every day stories and events by mothers, a bilingual Spanish interpreter was agreed to be adequate for the study. All of the interviews took place in the morning when 
most of the children were at school. Minimal disruptions occurred during the interviews; for example, a toddler was physically present for short time during one interview. This child added some normal baby commentary to the discussion until his mother could separate again from his reach. The home interviews, overall, went smoothly without interruptions; an occasional doorbell, phone, or a few distracting background sounds were encountered. For example, one home had a noisy washing machine running and another had a loud chirp periodically discharged, thought to be an expired battery from a smoke detector. Mothers' responses varied; therefore, the length of the interview ranged from slightly less than 1 hour to less than 3 hours. The average interview was $1 \frac{1}{2}$ hours. As the analysis progressed, a deeper understanding emerged from the maternal responses that initially did not call out to my attention.

\section{Data Analysis}

"Narrative analysis is the systematic study of narrative data" (Riessman, 2008, p. 7) where the story is the data. Riessman described various analyses methods. Thematic and structural are two such methods and both were employed in this study.

Riessman (2008) clarified that all narrative analysis is concerned with content, of the "what" (p. 53) of the narrative, regardless of whether it is written or visually presented. Content is the focus of thematic analysis. According to Riessman, thematic analysis is the most common data analysis method because of its straightforwardness and application across many settings. Keeping the story intact is a key difference with narrative analysis along with theorizing across stories rather than from within components of each story. The analyses process focuses on the "what is said" (p. 53), less on the how or the telling, in contrast to the "to whom" (p. 54) or "for what purpose" (p. 
54) typically detailed in structural or sociolinguistic methods. Thematic analysis entails the researcher work with one interview at a time, "isolating and ordering relevant episodes into a chronological biographical account" (p. 57); striving to preserve sequences and rich detail contained in long stretches.

In general, Riessman's (2008) process applied to all interviews in this study; analysis proceeded to identify underlying study aims in each story and naming (coding) them. According to Riessman, the researcher seeks to chart the landscape of the interpretative process that unfolds from personal stories and focuses on the telos, or an explanation of an occurrence related to "its purpose as part of its essence or nature" (Schwandt, 2007, p. 287). Thematic analysis assumed a case-centered approach, a fundamental difference in the analyses process. Particular stories (cases) were parsed to feature different patterns, ranges, and variation of the underlying themes and were then compared.

I transcribed the interview digital recordings as soon as possible to remain close to the data. The transcriptions were placed in a word document with three columns: two narrow columns on each side of one center column containing text form the interviews. Details and comments were entered in the side columns, such as background sounds that helped to (re)locate me in the same space while I read and reread the transcripts. Time points in the recording were noted for easier retrieval. Transcribed interviews were checked against the digital recordings by reading the interviews while carefully listening to the recordings. Corrected transcribed errors, nuances of language, pauses, words of emphasis and emotions were noted. For example, several mothers cried or were tearful as they retold specific emotion-laden events. Transcriptions were sorted in several cycles 
and reread many times. Three levels of coding were applied in the analysis process.

Thematic analysis. More specifically, thematic analysis was employed as the principle strategy. As A. Coffey and Atkinson (1996) suggested, the first coding level used the study's interview questions as a guide to organize the data. Initially segmenting data in this way allowed for identification of general and broad thematic content within the research question. Using this approach, it might seem as if the data related to one topic would be located similarly across narratives in response to a question. However, this was not the case; similar topics were not necessarily found bundled or located in the same spot of different interviews. The ability to locate data narrative segments around similar topics required multiple selective reviews. For example, different mothers provided varied depth and details in response to the study's initial open-ended request: Tell me your story of what it is like for you, as a Hispanic mother, to parent your child or take care of your child with CF. Several stories encompassed areas of interest the probes targeted.

To accomplish level-one coding, the responses were carefully read with attention to recurring language such as descriptive words, clauses, or statements that indicated common general characteristics (A. Coffey \& Atkinson, 1996). The second level of coding took on a more scrutinizing lens, became more complex, looking for details, coded as subcategories or subthemes. The data were reviewed for more detailed patterns, similarities, distinctions, and metaphors. This level of review looked for themes that represented the mothers' voice more closely. Therefore, direct words from the mothers were used when they stood out. Other subcategories were derived by my summary of what mothers stated. For example, the word hard was stated many times within and 
across stories; it was undeniably a subtheme. In this process, codes generated can overlap, intersect, or can be densely located in language or "nested" (p. 36). The same segment may be coded with more than one description or code. The final level used direct quotes to represent the themes and subthemes and to enter the final analysis. While coding is a successive process of drilling for representation of the mothers' data, there is not a common universe for it. A. Coffey and Atkinson contend that important data can be lost with coding. Therefore, the final step before analysis is to return to the "whole" (p. 46) narrative of what it is or might be.

In order to accomplish the interpretive process, the data must be organized to prepare it to be accessible for reading and exploring (A. Coffey \& Atkinson, 1996). I used matrices to organize the data in a Word document. This allowed me to explore the data by toggling back and forth, cutting and pasting quotes according to the linkages and relationships located. I reviewed the matrices as a whole with their similar themes and subthemes and also reviewed them in clusters. Clusters were separated by those originally in English and those in Spanish, and whose stories were told through an interpreter, to explore differences or similarities in patterns or themes.

While thematic analysis was applied to the majority of the storied segments, structural narrative analysis was applied to maternal responses, when mothers told their story around the time of diagnosis. The narratives were chosen because they explicitly represented stories' characteristics with a beginning, middle, and end, reflecting their personal experiences. An additional narrative also was analyzed with structural analysis.

Structural analysis. The selected storied segments were analyzed using Labov's (1997) model of structural elements to advance a deeper understanding of the narratives. 
Labov's approach identified six types of narrative clauses: a plot summary or what is this about? (abstract [A]); place, time, characters, and situation (orientation [O]); sequence of events (complicating action [CA]); the attitude of the narrator toward the complicating action (evaluation $[\mathrm{E}]$ ); resolution or result $(\mathrm{R})$; and coda $(\mathrm{CO})$, which brings the listener back to the present (Labov, 2002; Langellier, 1989; Riessman, 1989, 2008). Both the abstract and coda are optional in this model. In addition, Riessman (2008) asserts that while thematic analysis is often used for narratives, structural analysis might illuminate meanings or understanding not evident from thematic analysis.

In summary, analysis included both thematic and structural strategies. An iterative process beginning with the first interview and continuing after each session was employed. Within each interview, narrative stretches were sectioned off, as all content was not narrative. This yielded rich information for interpretation, a key element of this method. In addition, the narratives were interpreted, represented by direct quotes from mothers, to embody them as their own actors, presenting their voices directly. The final step involved member checking where two mothers confirmed the transcripts of their story accurately represented their voice (Creswell, 2012).

\section{Reflexivity: Investigators View}

According to qualitative tradition, assumptions and theories are applied in data analysis where the investigator ideally enters the study space with neutrality. However, it is more widely recognized that all investigators come to the inquiry space with subjectivity and interpretation without neutrality but also with their own personal life story (Marshall \& Rossman, 2011). Being mindful of these qualities and factors was an important awareness and stance as I entered the mothers' space and forged forward with 
this study. I am a white middle class American and align myself with a strong ethnic heritage, because of its presence in my everyday world as a second generation Italian. I am also a mother, reminded that Hispanic mothers might or might not align themselves with their particular heritage, gender, or different social class. The commonality of gender and motherhood might ground a relationship in the interview. When working and interviewing mothers from a different culture, sensitivity to unique culturally oriented expressions and descriptions must be acknowledged. In addition, experiences with the power relations of women navigating the mother role from a cultural perspective other than my familiar context and background could have created tension for missed significance from the mothers' stories. Riessman (1987) highlighted these issues and described experiences in which women interviewing women might not be enough. However, the experiences might hold less tension when a shared world view, such collectivism, is a more familiar contextual background. The presence of an interpreter for Spanish speaking only mothers was not only helpful for interpretation but also for the trust factor, also representing the Hispanic/Latina group

My interest in this study was stimulated from multifaceted influences and a lifelong career working with individuals who have $\mathrm{CF}$ and their families. Some of these dynamics included observed changes, and the sometimes growing tension with health care providers' approaches in the delivery of care to individuals with $\mathrm{CF}$ and their families, such as the dominant medical model and power relations; observations following relocation to a more culturally diverse geographic area; and the discovery of significantly poorer health outcomes reported for Hispanic groups as I continued to gain knowledge on the ever-changing aspects of CF. In addition, curiosity was motivated by a 
desire to investigate whether individual stories from mothers with children with $\mathrm{CF}$ differed from experiences reported from their dominant Euro-American counterparts. While the care and management of CF is influenced by current scientific progress, health care providers continue to grapple with issues, such as adherence and variances in health outcomes, independent of genetic mutations. Understandably, studies in the U.S. reflected a gap in representing individuals with $\mathrm{CF}$ of Hispanic origin as an exclusive and distinct group to study except when described in demographics as small subgroups within larger studies. This gap, in part, might be a result of the smaller number of individuals with CF represented in the Hispanic U.S. population when contrasted to more common chronic health conditions, such as asthma (Center for Disease Control and Prevention, $\mathrm{n}$. d.) or diabetes (Center for Disease Control and Prevention, 2014).

Accordingly, interests for this study emerged from my practice setting and from personal and professional reflection about the health care teams' interactions with the culturally diverse populations. Some examples are curiosity about what is known of culturally driven health practices; acknowledgment of the family's central role in the Hispanic culture, as discussed in the literature review as highly significant (Cortez, 2008; Gill-Hopple \& Brage-Hudson, 2012); and not the equivalent to patient family-centered care promoted in the current health care. The differences in world views of collectivism and individualism might be far less tangible covered beneath cultural layers, yet might have profound implications for individuals within the context of chronic illness. While current patient- and family-centered approaches to health care send a positive note for lack of bias, the patient and family experience of care delivery might be strengthened with increased culturally congruent care. 
Although this study recruited the sample from my practice setting, my role is primarily based in the acute care setting. I had no prior recent relationships with the majority of the study mothers. For example, mothers (M4 and M6) have a four year old daughter and a 17 year old daughter, respectively. I attended to them at the time of their diagnosis. Both have had few hospitalizations since diagnosis. Likewise, mothers (M8, and M9) have had medical follow up with their children in the outpatient setting. Last, mothers (M1, M3, M2, M5, M7, and M10) encountered hospital admissions with their children over the years, some more frequently and some more recently than others, but most children with CF have not been admitted within the past year except for two mothers (M7 and M10); one with her 9 year old son and one with her 8 year old daughter.

In addition, overall mothers' responses were genuine and open. I believe the mothers expressed openness about the health care team and system because of their relationships and trust developed with the CF team over time. Enduring relationships with the $\mathrm{CF}$ team might provide a foundation to approach and work with mothers and children in ways that might not yield meaningful discussions or similar outcomes with short term or intermittent medical encounters.

Finally, from the my own ethnic heritage, hearing stories about my grandparents' immigration, coupled with the parallel of family stories from the 19th century European immigration wave, sparked a curiosity to question what Hispanic families that live in the U.S. perceive if they have a child with $\mathrm{CF}$ and what do they experience and feel? Do Hispanic mothers who have a child with $\mathrm{CF}$ perceive themselves as a minority in the $\mathrm{CF}$ community, and what does that mean for the delivery of care? A growing sensitivity to the health disparities with underserved populations and a heightened awareness that 
Hispanic people are a minority in the CF community as well as nationally, stimulated concern. Last, my interest was inspired from stories of challenges, gifts, and sorrows endured by families, parents, young adults, and children who live with $\mathrm{CF}$.

\section{Trustworthiness}

Member checking is one approach to address the study's trustworthiness, akin to validity in the quantitative genre. It allows for transparency and feedback from the on the interpretation of data (Bloomberg \& Volpe, 2012). Member checking was done with two mothers: one with an interpreter and one was English speaking. Confidentiality and the voluntary nature of this interaction were emphasized. The purpose of the activity was explained along with an opportunity to clarify or change data to represent most accurately their voice (Holloway \& Wheeler, 2010). The English-speaking mother clarified a few minor points without changes to the overall story. The Spanish-speaking mother confirmed accuracy was represented. The interpreter also reviewed several interviews and confirmed accurate representation. An audit trial was kept to document and to support trustworthiness. Audit trails are an additional way to document and carefully detail data collection and decisions in the interpretative process. The advantage of thematic analysis from the narrated text is the ability to gain direct information from the mothers' unique perspectives, grounded in data (Hsieh \& Shannon, 2005). Finally, peer

debriefing with the dissertation committee facilitated credibility and trustworthiness by ensuring the analyses was grounded in the data (Marshall \& Rossman, 2011).

\section{Conclusion}

The study of mothers is integral to the study of children with chronic health conditions. Furthering the understanding of Hispanic mothers' experiences of parenting 
their child with CF and uncovering their (un)expressed needs within a sociocultural life space, assists to support better health care delivery and manage care more effectively and sensitively. Studying this ethnic group represents voices of Hispanic mothers' untold experiences, and gives back the respeto embedded in their cultural values. Understanding health experiences of a minority group situated within the dominant $\mathrm{CF}$ population also calls attention to potential entanglements that might have gone unnoticed and taken for granted and, therefore, needed interrogation. Through narrative analysis, a beginning space has been opened to inform nurses and other health care providers for future studies. Clarifying beliefs and experiences of mothers in their parenting role illuminates and directs development of nursing interventions and approaches toward more holistic, systematic life span planning.

In summary, this study explored and described perspectives of Hispanic mothers parenting children with $\mathrm{CF}$, their day-to-day living with $\mathrm{CF}$ management, their health beliefs, and health care experiences related to CF care through narrative stories. Research findings about CF flourish across many disciplines. However, this study concentrated solely on Hispanic mothers' perspectives within a subset of the CF community as a starting point to fill an existing gap not yet explored. The study represents maternal voices, muted from their sociocultural contexts. It adds perspective to their health care experiences and extends information for future health care planning consideration. This area of study merits further exploration. 


\section{Chapter 4: Analysis and Interpretation}

The analysis and interpretation strived to capture rich descriptions from Hispanic mothers' stories. First the study's sample characteristics are presented for both the mothers and their children with CF. The presentation of the study analyses first describes the narratives' contexts followed by exemplars of intact narrative units. Thematic analysis was the strategy for the majority of the narratives. The A. Coffey and Atkinson's (1996) and Riessman's $(1990,2008)$ work both guided the analysis process.

Thematic analysis, according to A. Coffey and Atkinson (1996), suggests a multiprong approach. Multiple successive readings were required to complete the process, which led to the creation of themes and subthemes. To accomplish the first level, readings explored general categories for coding. Attention was directed to phrases, clauses, and statements. I used the research questions to initiate this analysis, as these authors suggested. For example, the study question for aim 1 was used as a guide and directed codes related to role characteristics associated with maternal parenting. The second level required greater reading scrutiny. Careful examination was required to discover and uncover complexities, locating more details, which led to coding subthemes development. In this level, reading attended to selecting codes that most closely represent the mothers' perspectives. The final analysis prepared the data for interpretation by selective story segments parsed into quotes representing mothers' voices.

Select storied segments were further analyzed using Labov's (1997) structural elements model to advance the narratives' deeper understanding. Labov's approach 
identified six narrative clause types: a plot summary or what is this about? (abstract [A]); place, time, characters, and situation (orientation [O]); sequence of events (complicating action $[\mathrm{CA}]$ ); the attitude of the narrator toward the complicating action (evaluation $[\mathrm{E}]$ ); resolution or result $(\mathrm{R})$; and coda $(\mathrm{CO})$, which returns the listener to the present (Labov, 2002; Langellier, 1989; Riessman, 1989, 2008). The abstract and coda are optional in the model. The abbreviations are labels in the following narrative segments' presentation. Three main narratives were parsed into bounded segments around the diagnosis stories. Analysis was applied using strategies described by Riessman $(2008,1990)$.

The following study questions guided the analysis:

- What is the perspective of Hispanic mothers of parenting their child with CF?

- What are the health beliefs of Hispanic mothers related to the care of their child with cystic fibrosis?

- What are Hispanic mothers' perspectives of their health care experiences related to their child's CF?

Narrative, defined for this study, is the ordering of at least one or more events causally connected; story refers to the threads of plot and character woven together, the structure of which is designed to provoke an emotional response from the audience or reader (Paley \& Eva, 2005). These authors aptly summarized, "All stories count as narratives, not all narratives count as stories" (p. 85). Therefore, analyses were applied to select interview stretches that met the story criteria with a beginning, middle, and an end.

\section{Description of the Sample}

Characteristics of the mothers. The sample consisted of 10 mothers (see Table 2) with a median age of 32 years, ranging from 24 to 48 years. Nine mothers were 
married, one was single, never married. Two mothers worked outside the home, one worked part time. Maternal education levels ranged as follows: one completed eighth grade, one completed ninth grade, five completed high school, two completed community college, and one had 5 years of college in Central America. Four mothers did not recall having genetic counseling. Six mothers identified their country of origin as Mexico, two as El Salvador, one as Spain and one as Nicaragua. Six households spoke primarily Spanish in their homes; one mother reported both English and Spanish were used about equally. Three mothers brought their infants on the journey across the U.S. border to seek a medical diagnosis, treatment, or both.

Table 2

Sample Demographics: Mothers Characteristic

\begin{tabular}{lc}
\hline Age/years & Mothers $(\%) N=10$ \\
\hline $20-29$ & $2(20 \%)$ \\
$30-39$ & $3(30 \%)$ \\
$40-49$ & \\
Self-Identity as Hispanic* & \\
Country of Origin & $6(60 \%)$ \\
Mexico & $1(10 \%)$ \\
Spain & $2(20 \%)$ \\
El Salvador & $1(10 \%)$ \\
Nicaragua & $($ Table continues $)$
\end{tabular}




\begin{tabular}{|c|c|}
\hline Age/years & Mothers $(\%) N=10$ \\
\hline \multicolumn{2}{|l|}{ Language spoken at home } \\
\hline Spanish & $6(60 \%)$ \\
\hline English & $4(10 \%)$ \\
\hline Both & $1(10 \%)$ \\
\hline Requested interpreter & $5(50 \%)$ \\
\hline \multicolumn{2}{|l|}{ Education level completed } \\
\hline $8^{\text {th }}$ grade & $1(10 \%)$ \\
\hline $9^{\text {th }}$ grade & $1(10 \%)$ \\
\hline $12^{\text {th }}$ grade & $5(50 \%)$ \\
\hline Community college & $2(20 \%)$ \\
\hline College & $1(10 \%)$ \\
\hline Married with both parents in household & $9(90 \%)$ \\
\hline Single never married & $1(10 \%)$ \\
\hline \multicolumn{2}{|l|}{ Employment status } \\
\hline Does not work outside the home & $8(80 \%)$ \\
\hline Employed & $2(20 \%) 1$ part time \\
\hline Recall of genetic counseling & $4(40 \%)$ No \\
\hline Insurance: government funded & $100 \%$ \\
\hline
\end{tabular}

*initially identified from CFF Patient Registry, confirmed with participants

Characteristics of the children with CF. Characteristics of the children with CF were more diverse (see Table 3). Out of 10 mothers interviewed, six had daughters with 
$\mathrm{CF}$, age ranged from 4 years to 17 years; and there were 12 sons, ages ranged from 5 to 17 years. Six children were first born.

Table 3

Sample Demographics: Children Characteristic

\begin{tabular}{|c|c|}
\hline Age-years & Child with $\mathrm{CF}$ \\
\hline $0-5$ & $3(17 \%)$ \\
\hline $6-10$ & $8(44 \%)$ \\
\hline $11-15$ & $5(28 \%)$ \\
\hline $16-20$ & $2(11 \%)$ \\
\hline Total number of children of mothers & 18 \\
\hline Males & $12(67 \%)$ \\
\hline Females & $6(33 \%)$ \\
\hline Total & 18 \\
\hline \multicolumn{2}{|l|}{ CF diagnosed: } \\
\hline First born & $6(33 \%)$ \\
\hline Meconium ileus & $2(11 \%)$ \\
\hline New-born screening* & $2(11 \%)$ \\
\hline Prenatal diagnosis** & $1(5 \%)$ \\
\hline Sweat test & $18(100 \%)$ \\
\hline DNA analysis & $18(100 \%)$ \\
\hline
\end{tabular}




\begin{tabular}{lc}
\hline Age-years & Child with CF \\
\hline Siblings & $11(61 \%)$ \\
Child with CF with siblings with CF & $13(72 \%)$ \\
Siblings without CF & $1(5 \%)$ \\
Solo child with CF & \\
FEV $\%$ predicted & $13(72 \%)$ \\
$\geq 100 \%$ normal & $1(5 \%)$ \\
$100 \%-80 \%$ mild & $2(11 \%)$ \\
$79 \%-50 \%$ moderate & 0 \\
$49 \%-30 \%$ severe &
\end{tabular}

2 not documented

*two children had newborn screening with subsequent 7 siblings diagnosed; ** one child had prenatal diagnosis with a subsequent sibling diagnosed with $\mathrm{CF}$

In families that had multiple children with $\mathrm{CF}$, the youngest child was initially diagnosed followed with testing of the older siblings, resulting in $\mathrm{CF}$ diagnoses of multiple children in two families. One mother learned of her son's CF through prenatal testing, followed with subsequent testing of her older son and a later diagnosis of CF. Thirteen children had normal recorded lung function documented by forced expiratory volume in 1 second $\left(\mathrm{FEV}_{1}\right)$ of percent predicted; one was recorded in the mild range; two had lung function in moderate range; and another two had no recordings for two children because of their age. All of the mothers reported no smoking by any household member.

\section{Narratives and Analysis}

Six themes emerged from the interviews: (a) mothering, (b) growing and growth, (c) mother-talk, and (d) connected, (e) life disrupted, and (f) being here. Subthemes to life 
disrupted were (a) guarded optimism and (b) it's just hard; subthemes to being here were (a) parental gendered differences, (b) more mother talk, (c) education, and (d) communication won't happen without interpreters.

Interviews with three mothers were conducted at the hospital, at their request, where a private office was arranged. The remaining seven interviews were conducted in the mothers' respective homes. A private time without other family members present was arranged by each mother. During one interview, a toddler was with his mother for short time as the interview was winding down. When the interpreter was present, the three of us sat usually in close proximity. Water was offered and tissues were available if needed. The interviews were started in the same manner, first explaining the process, obtaining informed consent, followed with recording demographic information, and then proceeding with the interview by restating the first open-ended question. Taking a break during the interview was acknowledged. At the introduction, I attempted to put the mothers at ease by telling them that there were no right or wrong answers to the interview questions or suggesting to relax and just talk about their story. To protect confidentiality, all names have been changed and given a pseudonym; likewise hospital names or other locales have been given fictitious or generic names.

\section{Maternal Perspectives}

Not discovered until after the second interview, the seminal work of Sara Ruddick (1995), Maternal Thinking: Toward a Politics of Peace, partially informed the analysis. This work was introduced as groundbreaking when initially published in 1989, it is aptly described on the book's cover note as 'the first attempt to describe from a philosophical perspective, the thinking that grows out of the work mothers do". Ruddick's work 
evolved the scholarship of women's studies by distinguishing the practice of mothering separate from the institution of motherhood, from the underpinnings of patriarchal influence (O'Reilly, 2010). Ruddick maintained that three defining "maternal demandspreservation, growth, and social acceptability" (p. 17) - are achieved through committed maternal practice of "preservative love, nurturance, and training" (p. 17), comprising the work of mothering. Therein, she defined the work of mothering.

In the midst of the second mother's interview, as she described the conflicted worries between her role of being a mother and being a medical associate, an emotional tone surfaced that I labeled in the interview as "mother emotion." This descriptive, mother emotion, struck a deep chord within myself about the mothers. Serendipitously, I discovered Ruddick's (1995) work on maternal thinking. This body of work resonated with me as I reflected and proceeded through subsequent interviews. I noted a repeated strong sense of deep mothering qualities, which emerged as a recurrent theme.

Mothering. The following narrative segments exemplify the work of mothering advanced by Ruddick (1995). Three separate interview narratives are presented according to Labov's model $(1997,2002)$ around mothers' stories of their infants' CF diagnoses. Two infants were diagnosed during an acute hospitalization, a common presentation; the third was diagnosed at a later age in Central America. The analysis approach with the following narratives was conducted according to Riessman (2008).

Alba is a 24-year-old mother with her daughter 43/4-year-old daughter diagnosed with CF at 2 weeks of age. She has a 4-year-old son without CF. She is married and lives together with her nuclear family along with the maternal grandfather in a small cleanly kept urban apartment. Her husband is the primary breadwinner. Alba completed eighth 
grade. She is the full-time primary caregiver and does not work outside the home.

Spanish is the primary language spoken at home. She recalled having had genetic counseling. She related the following story of the time around her daughter's diagnosis (see Table 4).

Table 4

Alba's (M4) Story

\begin{tabular}{cc}
\hline Narrative Clause & $\begin{array}{c}\text { Structural } \\
\text { element }\end{array}$ \\
\hline
\end{tabular}

Section I.

1 In the beginning $\quad \mathrm{O}$

2 when I was first pregnant with her $\quad \mathrm{O}$

3 I was very happy $\quad$ EV

4 We, my husband and I were both very happy EV

5 we had been trying for a few months $\quad$ CA

6 and I hadn't been pregnant CA

7 and finally I was pregnant $\quad \mathrm{R}$

8 and umm we were - my husband was hoping for a girl CA

9 and I told him it would be a blessing of whatever is that we CA received

10 whatever God sent our way

11 and then I found out it was going to be a girl EV

12 and we were both very very happy EV 
Narrative Clause

Structural element

Section II.

13 and when she was born

$\mathrm{O}$

14 shortly after

$\mathrm{O}$

15 she developed a cold

$\mathrm{CA}$

16 and I brought her in multiple times

$\mathrm{CA}$

17 to try to get some medicine

CA

18 or something I could give her to help her get better.

$\mathrm{CA}$

19 However during multiple visits to the Dr.

$\mathrm{CA}$

20 and even to the hospital

$\mathrm{CA}$

21 I most times was told that she didn't have anything...

EV

Section III.

22 ...right before she was diagnosed

$\mathrm{O}$

23 she was very sick

EV

24 having a very difficult time breathing

$\mathrm{CA}$

25 I brought her into the hospital

$\mathrm{CA}$

$26 \ldots$ and they did tell me that their hopes that she would make it were

EV very low

27 and that was one thing that was very difficult

EV

28 It was very difficult

EV

29 because I was very happy

EV

30 that I was going to have a little girl

RE 


\begin{tabular}{cc}
\hline Narrative Clause & $\begin{array}{c}\text { Structural } \\
\text { element }\end{array}$ \\
\hline 31 and I finally had my little girl & $\mathrm{RE}$ \\
32 and this is what what happened. & $\mathrm{CO}$ \\
\hline Note $\mathrm{O}=$ orientation; $\mathrm{CA}=$ complicating action; $\mathrm{EV}=$ evaluation; RE = resolution; CO
\end{tabular}
$=\operatorname{coda}$

Alba was very soft-spoken. Initially, her affect was somewhat guarded, giving an impression of distance or caution. Her initial responses to the open-ended interview questions were brief. Probes were employed in an effort to connect and facilitate more openness, for her mother voice to emerge. For example, a probe introduced near the beginning of the interview asked her to talk about what makes the care of her daughter harder. She responded, "When I go back to my appointments...I feel like that some of the feelings that were present in the beginning start coming back" and then continued to talk more about her daughter. I asked her if she could talk a little more about the "feelings that were present in the beginning," she had mentioned earlier. Had I not probed further, it is doubtful that a space for the narrative would have been voiced; the probe opened the door. It was in that moment that the story emerged.

This story showed a simple, straightforward, yet formal, sequenced narrative with a beginning, middle, and end. In keeping with Labov's (1972) work, it demonstrated the elements of a story (section I) with the orientation of time (1), "in the beginning" and (2) the situation, "when I was pregnant." The complicating actions of "trying for some time to become pregnant" $(5,6)$ and an evaluation that described both parent's feelings of happiness, particularly because they learned the baby was female, just as they had wished $(11,12)$. There was no coda or abstract in the first segment. Significance of their situation builds with repetition $(3,4)$ and the use of intensifiers, such as very $(3,4,12)$ (Lieblich, 
Tuval-Mashiach, \& Zilber, 1998). The story continued (section II) with significant turns, "We had been trying for a few months, I hadn't been pregnant" and then she learned she was finally pregnant $(5,6,8,9)$ representing the sequence of events or complicating actions (Riessman, 1993). Last, the story moves the listener temporally forward. We learn about the pregnancy and about the baby's gender, followed with the birth to the first episode of illness, to repeated illnesses and unsatisfactory attempts to get treatment, and finally when the baby became very ill. These sequences answer the characteristic question, then what happened? The narrative clause in section III has the essential story elements with an added coda (32) signaling the end of the narrative, and "this is what happened." Codas "bridge the gap between the moment of time at the end of the narrative proper and the present" (Labov, 1972, p. 365). Codas bring the listener and narrator to the present, where they entered the narrative. In addition, while Alba was telling her story, her emotional tone was initially tearful and then she cried as she finished (27-32), adding to the deep intensity buried from 5 years in her past. The story continued (see Table 5).

Table 5

Alba's (M4) Story Continued

Narrative Clause

Structural

Section IV.

33 And when she [daughter] was diagnosed

34 she had a pretty long hospitalization

$\mathrm{CA}$

35 it was about three months.

$\mathrm{O}$

36 When I first learned about all the treatments

$\mathrm{CA}$ 
Narrative Clause

37 and what I would have to do to take care of her

Structural element

38 and everything that she needed

$\mathrm{CA}$

39 I felt it would be very very difficult

EV

40 and I didn't know what to do.

$\mathrm{CO}$

Note. $\mathrm{O}=$ orientation; $\mathrm{CA}=$ complicating action; $\mathrm{EV}=$ evaluation; $\mathrm{RE}=$ resolution

The story segment ended describing a lengthy hospitalization with expressed fears about the difficulty of her infant's care and her ability to take care of her new baby girl. The coda (40) brings the listener in this moment back to the present.

Sophia, is a 34-year-old mother of Luis, 9 years of age, diagnosed at 2 months of age. He has a younger brother and sister without CF. Luis' hospitalizations have become more frequent in the past several years. They reside in a large well-kept home in a suburban neighborhood. The family lives together without extended family members. Sophia described her role as a traditional, domestic work-at-home mother. Spanish is the primary language spoken in the home. Luis's father works full-time and is the primary breadwinner. Sophia completed 12 th grade; she did not recall having genetic counseling after her son's diagnosis. She relates the following story (see Table 6):

Table 6

Sophia's (M7) Story

\begin{tabular}{ll}
\hline Narrative Clause & $\begin{array}{l}\text { Structural } \\
\text { element }\end{array}$ \\
\hline Section I
\end{tabular}
Section I.

1 At first when he was born 
Narrative Clause

2 he was born fine, Structural element

3 there was no problems... $\quad$ EV

4 he looked healthy... RE

5 ...After the first week $\quad \mathrm{O}$

6 he was fine at home... $\quad$ EV

7 and in the beginning of the third week $\quad \mathrm{O}$

8 He started having fevers $\quad$ CA

9 and that's when I took him to the emergencies... CA

10 ...he was just for three days on antibiotics $\quad$ CA

11 and after that we went home. RE

12 In the first month $\quad \mathrm{O}$

13 that's when I started seeing more diarrhea... CA

$14 \ldots$ I give him the bottle he would just vomit it... CA

15 .... So he started dehydrating CA

16 we brought him in to the first hospital... CA

17 He [doctor] just told us we'll just watch for him. RE

18 And then after that I took him to the hospital [different hospital] $\mathrm{O}$

19 ...They didn't do any tests, only blood tests. CA

20 and they say everything came out good, EV

21 so just go home $\quad$ CA

22 but he didn't stay. RE 
Narrative Clause

Structural

23 So I did, as a first mom element

24 I didn't know what to expect

$\mathrm{CA}$

25 or what to do

$\mathrm{CA}$

26 or what else.

$\mathrm{CA}$

27 So I took him to TJ in Mexico

RE

28 So there they give him a lot of medicine...

$\mathrm{CA}$

29 but it's just, it didn't help...

EV

30 ...I took him to the pediatric

CA

31 in that same month

$\mathrm{O}$

32 and he say he was losing weight

EV

33 So he [pediatrician] just changed the formula...

$\mathrm{CA}$

34 So it wasn't it wasn't just helping...

EV

35 So one night I just had him getting smaller and skinnier

$\mathrm{CA}$

36 and I didn't see him, like good...

EV

37 ...we just decided around 11 in the night

$\mathrm{CA}$

38 we took him to where he was born in hospital...

$\mathrm{CA}$

39 So they recommend straight to the local children's hospital

RE

Section II.

40 So that's when everything started...

41 When we got there

$\mathrm{O}$

42 he was really bad looking

EV 
\begin{tabular}{cc}
\hline Narrative Clause & $\begin{array}{c}\text { Structural } \\
\text { element }\end{array}$ \\
\hline 43 really bad... & $\mathrm{EV}$ \\
$44 \ldots$ and then it [sweat test] came out positive... & $\mathrm{EV}$ \\
45 and that's when everything started & $\mathrm{CO}$ \\
46 You know what, now we know how to treat him & $\mathrm{RE}$ \\
47 He'll be getting gaining his weight back & $\mathrm{RE}$ \\
48 he's just, we'll know how to treat him & $\mathrm{RE}$ \\
49 and and so that's when everything changing for him & $\mathrm{CO}$ \\
\hline Note. O = orientation; CA = complicating action; EV = evaluation; RE = resolution; CO
\end{tabular} $=\operatorname{coda}$

The story above portrayed elements of a narrative, moving the listener temporally forward. The resolution and coda signaled a positive feeling $(48,49)$. Both of stories told by first-time mothers presented similar sequences: they both described a healthy baby at birth and then, in the immediate newborn period, both infants had multiple illnesses requiring medical attention. Similar to Alba's story, Sophia draws the listener in, moving forward temporally and depicting more clinical details to the scene of what happened. Turning points in the story were specifically detailed, for example, deciding to go to the hospital late in the night $(37,38)$ conveys both an urgency and fear this mother perceived when viewing her infant as "getting smaller and skinnier and didn't see him good" (35, 36). While "getting smaller and skinnier" described her infant's appearance, it also represented a metaphor that described this mother's feeling of loss of control, losing the capacity embodied in her protective maternal role. The experiences of these first-time mothers confronted with the vulnerability of their sick infants suggested that protective actions are reflective of maternal role transition and maternal subjectivity as a means of 
knowing (Belenky, Clinchy, Goldberger, \& Tarule, 1986). Belenky et al. developed a typology of ways women learn, know, and value what is unique to them. Belenky et al. defined subjective knowing as "knowledge conceived as personal, private, and subjectively known or intuited" (p. 15). In this story scene moment, the subjective, intuitive learning, and maternal knowing mode unfolds. The mothers protected and preserved their infants' lives contrary to the unsatisfactory medical information received. Ana, is a 33-year-old, single mother. She emigrated from Central America to the U.S. in her early 20 s to earn money for her daughter's medical care. Isabel, her first child, is 14 years old. During her early years, she stayed behind and was cared for by her maternal grandmother. She was reunited with her mother at the age of 7 . They had not seen each other during their 4 years of separation. Ana remarried when Isabel was 8 years old and has a 6-year-old daughter. The family lives together in a residential neighborhood with no additional extended family. Spanish is the primary language spoken at home. The interview was at her home, a very simply furnished space decorated with photographs of her girls. Ana completed $12^{\text {th }}$ grade and recalled having genetic counseling after her daughter was diagnosed at almost 4 years of age. She related her story (see Table 7):

Table 7

Ana's (M9) Story

Narrative Clause $\begin{aligned} & \text { Structural } \\ & \text { element }\end{aligned}$

Section I.

1 Initially, when she when she was born

$\mathrm{O}$

2 everything seemed to be going normal,

EV 
Narrative Clause

Structural

element

3 she was gaining weight as expected

EV

4 and when she was about three months (I)

$\mathrm{O}$

5 she started developing a cough

6 and she was getting very sick.

CA

7 We are from a small town,

$\mathrm{O}$

8 so in order to get to a doctor

9 we needed to travel to another town

CA

10 which was about six hours away.

$\mathrm{O}$

11 When I brought her to the Dr.

12 the first initial visit to the pediatrician $\mathrm{CA}$

13 they diagnosed her with pneumonia (I)

14 and they said she was very sick EV

15 and they had to admit her to the hospital. $\mathrm{CA}$

16 Then, she went back home. RE

17 She was okay. $\quad$ EV

Section II.

18 And it was not long after

$\mathrm{O}$

19 she started getting sick again

20 and developing more cough

$\mathrm{CA}$

21 and had her take her back to the Dr.[same Dr.],

22 once again

$\mathrm{O}$ 
Narrative Clause

Structural

element

23 and was admitted to the hospital.

$\mathrm{O}$

24 Once again

25 they said she had pneumonia.

$\mathrm{CA}$

26 It was very difficult for us

EV

27 because she was getting very sick

$\mathrm{CA}$

28 all the time

29 even with her diagnosis of pneumonia,

$\mathrm{O}$

30 it didn't seem like they were helping her get better (I). 16:27

EV

Section III.

31 When she was about three years old,

$\mathrm{O}$

32 was when the doctors started to tell me

33 that she possibly had cystic fibrosis (I).

CA

34 They sent in a sweat test

35 which confirmed it. (I)

CA

36 However, they didn't have the medication and treatments

37 necessary to treat cystic fibrosis,

38 so it was very difficult for them to care for her.

39 The doctors didn't have the medications necessary

EV

40 and everything was very expensive.

EV

41 So my mom and I

CA

42 were kind of discussing our options 
\begin{tabular}{ll}
\hline Narrative Clause & $\begin{array}{l}\text { Structural } \\
\text { element }\end{array}$ \\
\hline
\end{tabular}

43 and we decided for me

44 to come out to the US to work...

RE

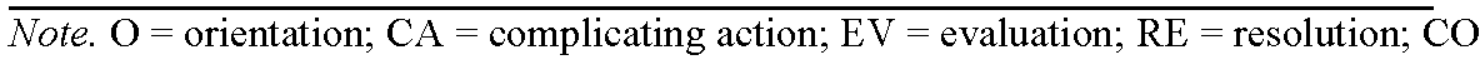
$=$ coda

Ana's story highlighted her newborn infant's similar illness trajectory. Parallel to the first two stories, the trajectory mirrors the initial maternal observations. However, Ana's story contrasts a background of living in a developing country where families traveled long distances for medical care (8-10) with limited access to specialized medical care (38-40). Her story unfolds and moves the listener forward in time from birth through repeated pneumonias, hospitalizations (13-15, 23-29) and finally her daughter's diagnosis around 3 years of age (33-35). The repetition of events of Isabel's illnesses, "taking her back and forth to the doctor, once again" and "admitted to the hospital, once again" (21-24) tells of the cyclical nature of chronic conditions disrupted with acute events and followed with short periods of stabilization. The story imparts the feeling of being worn down by a pattern of illness without resolution, without answers.

Ana's story depicted a striking departure of her daughter's illness trajectory from birth to diagnosis contrasted with Alba and Sophia's stories. Alba and Sophia resided in a large metropolitan area with access to cystic fibrosis centers, where facilities for comprehensive diagnosis and treatment were available. CF might be underdiagnosed in geographic regions, such as Latin America, where limited health care access, limited facilities, limited specialists, and limited resources exist.

Each mother's story concerned not only her infant's diagnoses but also her experience as a first-time mother. Similar patterns of the telling of their stories take the 
listener through a journey of personal and life-changing moments in time: initially having a normal baby, gaining weight, then of the repeated respiratory illnesses requiring immediate medical interventions, and yet with continued and recurrent cyclical symptoms typical of many CF presentations. The linear chronological fashion in which each mother retold her story offers a space for mothers to reconstruct and make order and sense out of what is often a chaotic disorienting experience (Riessman, 1990) culminating in a diagnosis that is mostly unfamiliar or unknown. While the similarities of the infants' progression of illness presentations were not unexpected, the emotional tone and mother's affect varied. Alba demonstrated a high level of emotionality when relating her experience surrounding the diagnosis of her now almost 5-year-old daughter. Her coda "and this is what what happened" (32) suspended her story, bringing her sadness into the present. This contrasts with Sophia's resolution, "he's just, we'll know how to treat him" (48) where hope and optimism punctuated the point of the story depicting a sense of maternal relief for her infant's illnesses. Last, Ana suspended her story with no resolution or coda, not knowing how exactly she proceeded with plans to come to the U.S. to support her daughter's care. There was less emotionality in Ana's story, perhaps because she distanced herself because of the monumental decision to relocate to the U.S. This might have loomed large over the immediacy of Isabel's health condition, which became a familiar cyclical pattern. Ana might have suppressed a probable painful memory of separation from her daughter. She stated later:

It's very typical of mothers to come over to the U.S. to work to help care for their children, but of course, they leave them behind in their hometown. That's very 
common, I guess what they don't realize is the harm that they are doing to their children, leaving them at such a young age.

Applying Riessman's (2008) structural analysis to the above narrative segments opened a deeper meaning from the maternal voices that otherwise might not have been exposed by thematic analysis alone.

Developmental transitions underscore the vulnerability wherein children and mothers are challenged to master each developmental stage. Mothering and protection are not limited to infancy but extend across developmental stages. As one mother (M1) with three sons with CF related, "I think it $[\mathrm{CF}]$ puts an element of worry in the back of your mind all the time that most parents can shake off." This mother expressed feeling acutely aware of the various $\mathrm{CF}$ potential complications or problems that require monitoring within her mothering capacity to assure her children's health remains stable. These vulnerabilities go beyond normal childhood development. They become part of what mothers do, albeit conscious and, at times, relatively less conscious, actions embedded in their work of protection and nurturance.

The preceding stories illustrate the complexity of life transitions, including developmental, situational, and health and illness, consistent with transition theory (Meleis, 2010). The transition from a nonparent to parent status is one of life's most significant transitions, encompassing all spheres - emotional, physical, spiritual, and psychosocial — within the a culturally defined context. The situation of being a new mother demands major shifts in interpersonal and personal identity of private personal spaces of the individual dyad. With a new birth, a new triad, typical in a nuclear family, requires new relationships that might introduce a dynamic of unanticipated tensions. 
Consistent with other studies (J. S. Coffey, 2006; Nelson, 2002), adding a diagnosis of a chronic condition catapults a new parent into a myriad of emotions such as fear, anxiety, injustice, disorientation, grief, denial, shock. A genetic condition might also bring about additional feelings of blame and guilt (Henneman, Kooij, Bouman, \& ten Kate, 2002; Rowland \& Metcalfe, 2013). When the onset or diagnosis of a condition corresponds with an individual, family, or life-cycle transition, the experience might be amplified (Rolland, 1994).

Alba's response to the probe question about "when I go back to my appointments...I feel like that some of the feelings that were present in the beginning start coming back" called to mind the growing interest of posttraumatic stress symptoms associated with chronic conditions. Posttraumatic stress is characterized by symptoms that do not meet all criteria for posttraumatic stress disorder. Cabizuca et al. (2010) reported that approximately $63 \%$ of parents of children with $\mathrm{CF}$ reexperienced intrusive recollections or psychological distress surrounding the diagnosis and care of their child with CF. For Alba, her experience of happily anticipating motherhood associated with her maternal role transition, then followed with the life-threatening event of her very sick newborn infant, might signal her as an at-risk mother for posttraumatic stress symptoms. This phenomenon is closely aligned with pediatric medical traumatic stress studies. Kazak et al. (2006) found that the severity of illness was not correlated with posttraumatic stress disorder symptoms in parents of children with cancer.

The elements of Ruddick's (1995) framework require a commitment to meeting the infant's demands through preservative love and protection, evident in the above narratives. Preservative love involves maternal recognition of the infant's fragility and 
vulnerability. This maternal action is contrasted with other actions such as maltreatment, indifference, or running off, essentially not embodying the maternal role as a responsible commitment. This first element is foundational to maternal thinking and the work of mothering. While the concepts are derived from seemingly day-to-day repetitive routines of what mothers do, they are carefully distilled to incorporate judgment, reflection, and emotion, unifying maternal thinking (Ruddick, 2007). Through a maternal and feminist lens, Ruddick's work brings to light the exceptional capacity and agency translated to mothering contrasted with the dominant patriarchal view of historically devalued roles.

The mothers in the narratives, similar to the other study mothers, projected an ethos of care. All of the mothers, of which seven were first-time mothers, realized the vulnerability and fragility of their infants, consistent with Ruddick's description. The mothers were from diverse walks of life, yet were driven to seek help to protect and care for their infants. Three risked crossing continental borders from Central America to the U.S., motivated to save their infants and preserve their lives. Several mothers repeatedly traveled to multiple emergency rooms, multiple times, until their infants became so ill they required hospitalization. In Ana's (M9) situation, the prolonged back-and-forth physician visits and hospitalizations might have resulted from the country's limited health system. It is unclear if her daughter's delayed diagnosis was a result of limited resources, the rarity of $\mathrm{CF}$ diagnosed in her country, or an atypical presentation. However, these mothers persisted in their search for answers despite barriers and disparities, each time sent home without an answer. Simultaneous with the transition into their mother role, they were shifting into a new way of knowing, a maternal way of knowing, finding their maternal voice until they found themselves at a juncture that 
cleared their path to their infants' diagnoses. Ruddick (1995) writes of the universality of some features of mothering. I would argue that this element of mothering is universal.

Growing and growth. Ruddick's (1995) description of fostering growth and nurturance in children is a second distinct element in her framework, to nurture the unfolding and expanding spirit of the child. While this aspect of mothering is distinct in the "maternal thinking" (p. 83) framework, it has overlapping qualities with both preservative love as well as training. Growth extends to all aspects of the child's being, encompassing the spirit of the child. Nonetheless, in this framework, nurturance is more relevant to the judgment mothers continually exercise to promote holistic growth or support decisions and actions. The mothers related narratives that represent nurturance and how their care had evolved over time. Some of the mothers compared the care of their child with CF to their well siblings.

Alba (M4), the mother of a 4-year-old daughter, stated:

When I first learned about all the treatments and what I would have to do to take care of her and everything that she needed, I felt it would be very difficult and I didn't know what to do.... It's a little bit different [now] as far as her treatment and taking care of her. Otherwise it's almost the same... and I take care of them [including her son without $\mathrm{CF}$ ] the same.

Teresa (M11), the mother of an 8-year-old daughter, stated:

It was hard because they gave me a lot of medication and I didn't know how to give it to her because everything supposed to have a way to give them to her.... She doesn't fight me anymore with her treatments or taking her meds. Yeah, that's the best thing because she used to fight me. 
Alba's description moved toward a more typical way to take care of her child. Where once she was fearful, she has moved to seeing her daughter and son's care as not so different. Teresa's school-age daughter called attention to similarities with other mothers: overwhelmed with their initial care. Over time, her daughter's management grew to be less distressing. Treatments are a life connection for $\mathrm{CF}$, however, they do not always follow the pattern of becoming less problematic with age as depicted below. Sophia (M7), the mother of a 9-year-old son, stated:

He was the first one, we did know how to do his treatments and the time... when he was a baby it was easier for me, I mean 'cause I'd just grab him in my arms and then I'll do the pounding...I try to have the same rules...to have the same attention for the three of them....Yeah, I'll do the same as with my other two children.... I mean I don't want him to grow up and think "and me, no, I can get away with everything"....He wants to move more around and he questions me, "like why do my brother and sister get to play and I have to go upstairs and put my treatments?"

Estela (M5), the mother of a 14-year-old daughter, stated:

She...needed a lot of care.... The one thing that is easier for me now that she older and she is able take a little bit more responsibility with her care she's very disciplined...I've been giving her a little more independence I'm very careful because of all her needs and because of her age. Because of her age it's most difficult.

Ana (M9), the mother of a 14-year-old daughter, said, "Sometimes mothers are very overprotective... let them live as normal as possible, even with their illness and not be too 
overprotective and not feel sorry for them and to encourage them to live a more normal life."

The final three preceding dialogues, Sophia (M7), Estela (M5), and Ana (M9), depict the mutable challenges that develop with the transition from childhood to adolescence. Mothers' descriptions of care changed as their children's development progressed: Sophia's son started questioning why he has treatments, while Estela's daughter's care became easier as she assumed some self-care responsibilities and wanted more independence. Ana's is mindful not to be too overprotective, to try to live normally with CF. Inherent with older children are the joys of growth and a wider circle of influence. The work of mothers involves teaching, discriminating, and determining the safety of their expanding worlds. As children grow, mothers must also grow in understanding and in flexibility. The antithesis can also exist where mothers might become domineering (Ruddick, 1995). As the adolescent seeks independence, mothers' control and protection, whether conscious, superimposed with CF might, impede a child's psychosocial and emotional growth. The Hispanic cultural stereotype of overprotection ascribed to females contrasted with males as more privileged is well described (Chang \& Liou, 2009; Koss-Chinino \& Vargas, 1999; Updegraff \& Umana-Taylor, 2015). These cultural traditions and values potentially influence mothers' responses to gendered developmental challenges.

Several mothers commented on how they "stay on top of the treatments" as a strategy to keep their child healthy. Debora (M8), mother of two sons with CF who have never been hospitalized related: 
In my experience, I am scared not to do a treatment because it might have a negative impact on their health. For me, my priority and my main thing is for caring for my kids; that's the most important thing for me.

Maternal voices reflected the element of nurturance expressed from feeling connected to their children, to being there for their children, to telling detailed reviews of daily CF care routines, treatments, and attention to nutrition, required of $\mathrm{CF}$. Their voices reflected their individual growth toward a greater understanding of the concerns, conflicts, and demands of their growing children. Reflective maternal growth, in turn, stimulates new ways of maternal knowing.

Mother talk. The mothers expressed narratives that represented the training element of Ruddick's (1995) framework. This element prepares the child for social acceptability. Children require training, guiding, correcting, disciplining as a form of teaching, and learning, as universal expectations. However, the process of training provokes controversy, embedded in social, cultural, and family histories. While Ruddick's work focused on well children and training, it has relevance for children with special health needs. My interpretation in this section extends this element beyond the socially acceptability to what is developmentally necessary for children's growth toward independence.

The mothers repeatedly described talking to their children as a training-teaching approach. As developmental challenges emerged, mothers were confronted to respond thoughtfully to help their children make sense out of their life experience with CF. This is depicted in the following storylines, illustrating their talking about the importance of treatments and commitment to perform the prescribed $\mathrm{CF}$ care. 
Alba (M4) stated:

...also sometimes Dolores [4-year-old daughter] has a hard time so that kind of makes it a little more challenging but I try my best to explain to her why it is that she has to receive treatments and explain to her what it is that she has and how to help her keep her healthy.

Debora (M8) stated:

Also I try to talk to the boys and explain to them as much as they can understand what they have with their illness. My 7-year-old, Mario, is able to verbalize that he has cystic fibrosis and he knows his lungs have some problems or he's sick from his lungs.

In that aspect it's a little difficult...that's when they [two sons] might not want to comply with their treatments as much.... That's when it becomes more difficult, and that's when they start questioning a little bit more why the other kids don't have to do it.

Sophia (M7) stated:

So it's a little challenging for me and for him, but yeah that's why I try to talk to him every day you know... and then talk to him every day. I mean he's growing up, he's growing up. And have him know why he has to, why it's important for him to take care of himself.... So, yeah it's challenging but he knows he has to do it...like there's a lot of different medications for different diagnosis, like when you get sick and then that's what I tell him.

In the storylines above, the children were a little younger, yet mothers engaged them early in their care, committed to their child's well-being. As children progressed 
toward adolescence, maternal strategies changed to meet the challenge of training demanded of the respective developmental stage. Two mothers with adolescent daughters spoke. Estela (M5) stated:

So now that Natalia's [14-year-old daughter] is older, I still stay on top of her, make sure she's doing all of her treatments. However it is different, and it's a little bit easier because before she was very small and I had to do everything for her and she used to cry a lot or it was harder for her to express about her needs.

She later shared the following that her daughter voiced:

She sometimes expresses to me that her life expectancy is about 30 and so she kind of has a worry about that, but I tell her that there's other cases where individuals have lived up to 40 even 60 or longer. I explain to her that science is advancing and maybe in the future there's going to be better options and treatments that will help with life expectancy but also for comfort.... So I just tell her also we don't know when our time is, we'll all be leaving, but if you take care of yourself and do your treatments, then you have better possibilities.

Estela described her life as being easier since her daughter has shown more responsibility with CF self-care with age; implying it was harder when her daughter was younger. The continued maternal supervision illustrated that training employed in the practice of mothering applies to all ages yet requires different tactics. Older ages bring distinct challenges, such as talks about a shortened life expectancy. Such topics go beyond, what most would agree to be, anything but a common adolescent motherdaughter conversation. This conversation elucidates the challenges of motherhood, to use Ruddick's term, the intellectual activity, required in addition to physical and emotional 
training and growth. Maternal thought is reflected in a mother's ability to respond to such personal and emotional talk. Mothering chronically ill children entails learning how to talk with them about sensitive topics raised by their children, talks that most mothers in mainstream society will never need to approach.

Estela contrasted mothering as harder when her daughter was younger and continued to talk:

So some of the problems that some mothers' experience, one of the important ones is parent education. Sometimes I'm not having the highest level education [when clarified she stated she meant she did not have the best understanding of $\mathrm{CF}$ ] or not knowing, and also one problem, that was not accepting the diagnosis I was given [in Central America] and trying to prove to the doctor that the weather could possibly have something to do with it [her recurrent CF illness]. So I took chances and took risks and came out here to get more information, find out if that was really what she had because I doubted that was what my daughter had.

Estela described the denial state she entered when informed about her daughter's CF diagnosis in her home country. She emphasized that a mother also needs training and education to understand the requirements of care to fulfill maternal role functions. Her comment suggests that her denial influenced her initial understanding and training receptiveness. This point is also evident from the following mother:

Ana (M9) stated:

Also with the stage that Isabel is in, that's definitely an area where I could have a little more help, helping them better understand their illness and better 
understanding how they feel and how they view themselves like in comparison to other healthy peers.

Ana reflected on the ongoing training that mothers likewise must acquire to respond with thoughtful maternal guidance. Ana told more about what else her daughter expressed, "At one point recently she shared that she doesn't feel like she's not gonna get past high school so, I kind of live with that fear that something might happen to her."

Mothers recognized the importance of educating or training their children across all ages. They also acknowledged gaps in their personal sphere of mothering that might be edified.

Connected. Several mothers requested clarification of the question related to the practice of their day-to-day selfhood, what gets them through their day? Some responded with an initial pause. Their pause suggested that they did not know how or what to answer because they either had not been presented with a question, as such, or had not reflected on this aspect of self. Their responses were child centered, related to what most parents might take for granted every day, that their child will wake up and proceed with their typical day's activities. The majority of responses from mothers related a deep gratitude and appreciation for their child just to awaken or to just be in their world. After leaving the fourth interview, I was struck by a feeling, a sense of the depth of care and commitment the study mothers evoked in the everyday care of their children with $\mathrm{CF}$. As interviews progressed, I discovered Ruddick's (1995) work, and it called to mind that feeling mothers imparted about their day-to-day mothering (see Table 8). 
Table 8

Ana's (M9) Story

\begin{tabular}{llc}
\hline Narrative Clause & $\begin{array}{c}\text { Structural } \\
\text { element }\end{array}$ \\
\hline $1 \quad$ I always feel motivated & in the morning \\
3 & when I see her get up, & $\mathrm{O}$ \\
4 & and she's awake and walking & $\mathrm{CA}$ \\
5 & and start getting ready, & \\
6 & that definitely helps me through my day. & $\mathrm{EV}$ \\
7 & Seeing her & $\mathrm{R}$ \\
8 & get up in the morning, & $\mathrm{R}$ \\
9 & getting up in the next morning & $\mathrm{R}$
\end{tabular}

Note. $\mathrm{O}=$ orientation; $\mathrm{CA}=$ complicating action; $\mathrm{EV}=$ evaluation; $\mathrm{R}=$ resolution

The bound narrative segment above follows the pattern of plot Labov (1997) outlined, with an orientation of time, followed with the sequence of events that moves forward in time, then provides the evaluation of her story. The analysis using this form lent a different perspective to the story. Although lines 3 through 5 speak of typical activities, gets up, she's awake, walking, getting ready, they metaphorically represent life, and she's alive each morning. The use of repetition in the resolution of lines 8 and 9 underscored the gravity this seemingly simple, ordinary narrative plays out in Ana's world (see Table 9). 
Table 9

Ana's story continued:

Narrative Clause

Structural

10 In the past,

$\mathrm{O}$

11 I guess because of my own fears,

EV

12 I would sometimes

$\mathrm{CA}$

13 go in the middle of night

$\mathrm{O}$

14 and wake her up

CA

15 and just to make sure she was still okay.

$\mathrm{R}$

Note. $\mathrm{O}=$ orientation; $\mathrm{CA}=$ complicating action; $\mathrm{EV}=$ evaluation; $\mathrm{R}=$ resolution

The narrative segment continued to reflect elements consistent of a story. The mother expressed ways she coped with her fears by vigilance, described in the literature as a known means of coping (J. S. Coffey, 2006). There is no abstract or coda presented; however, the bound narrative of these two segments highlighted the child-centered practice of mothering, recognizing her daughter's vulnerability and determination to keep her safe. Other mothers responded similarly:

Debora, the mother of two sons, ages 5 and 7 years old, said, "seeing my kids grow and know that they're okay right now also helps me get through it and now that is what my life is about just getting them to grow up and stay as healthy as possible."

Lucia, the mother of three sons, ages 6,10 , and 11 years, stated:

I would say it used to be in the beginning, faith. Now it's kind of almost an attitude, it's just us against the world...that we just really stick together and it's all about us and about the kids and that's what keeps me going. 
Gloria, the mother of a 17-year-old daughter, stated:

The thing that helps me the most getting through every day is getting up and seeing my daughters becoming more independent and doing what they wanted do and that's what I usually look forward to every day. Just doing whatever it is that my daughters have coming up and plan.... I feel very connected to my daughter and everything my daughter experiences. I, in some way, experience it myself as well. When my daughter is sick, I feel sick or when my daughter is sad, I feel sad. One mother talked about meditation as a way she recharges and takes care of herself. The mothers underscored the child-centric focus, irrespective of the child's age, as central actors in their maternal world. The work of mothering was captured as continuous threads of maternal discourse, nurturing, and preservative love: "seeing my kids grow," "getting them to grow up and stay as healthy," "seeing my daughters becoming more independent and doing what they wanted do," and "it's all about us and the kids." Mothers spoke about the relationship they held with their children, seeing them grow, and delighting in their independence becoming a measure of their maternal success. According to Gilligan (1993), "women not only define themselves in the context of human relationship but also judge themselves in their ability to care (p. 17). Gilligan's contributions of the psychological development of women outlined differences from commonly learned maledominant theories, such as those of Piaget, Kohlberg, and Freud. Her work brings to light differences in gendered psychological development. Women develop and relate to the world through connectedness, relationships, and communication. Mothers' relationships with their children change over time; they develop a strong connection through caring. Hispanic motherhood has been described as a life goal (Chang \& Liou, 2009) portrayed 
as self-sacrificing and devoted. As primary caregivers, these authors found that mothers developed closer relationships with their children, more so than with fathers. Through close relationships, mothers gain a way of knowing that imbues competence required to care for their children, and new ways of knowing are constructed with the CF care.

Through a critical feminist lens, Hispanic mothers' roles might be reframed as those who choose the responsibility of mothering and derive meaning from their children in their own reality. Meaning is consequential from critical reflection. Critical reflection, in turn, provokes growth. Growth extends not only to understanding physical demands, but becomes entwined with the complexity of the holistic being; reciprocal relationships are activated.

Ruddick (1995) defined mothering as a discipline. She likened the discipline of mothering to that of a scientist,

...writing up her experiment, a critic working over a test, or a historian assessing documents," ... She asks certain questions - those relevant to her aims - rather than others; she accepts certain criteria for truth, adequacy, and relevance of proposed answers; and she cares about the findings she makes and can act on. (p. 24).

Life disrupted emerged as a major theme with two subthemes: (a) guarded optimism and (b) it's just heard.

Life disrupted. Guarded optimism. The majority of mothers addressed health as a major concern when talking about how they perceived their child's future. Gloria, the mother of a 17-year-old daughter, said, "I would love for her to accomplish her goals of 
becoming a psychologist, but the other main thing is making sure she stays on top of her own health and hopefully have a longer future and a healthier life."

Sophia, the mother of a 9-year-old son, said, “...be healthy, like working for him as much as I can, for him to be healthy for him not to be admitted as much."

Marta, the mother of six sons with CF, said, "Keeping positive. I think they're doing good. I don't think the CF is going to hold them back to do anything. I see them wanting to be someone in the future."

Debora, the mother of two sons, said, "I hope they have as good as health in the future as they do now. But the doctors say in the future there could be complications and they never really know."

While some expressed positivity as above, some talked also about their fears punctuated with uncertainty. Lucia, the mother of three sons with $\mathrm{CF}$, stated:

I wonder what their health will be like, you know, as they grow older and that worries me that they're gonna feel insecure or feel unloved because people are gonna shy away from them because they don't want to be their support system. The fact that knowing realistically I can't always be there for them as they get older, that scares me.

Ana, the mother of a 14-year-old daughter, stated:

When I think about her future, I am sometimes afraid of what the future will bring, I know this illness it's kind of like you don't really know, there's no cure and she can continue her treatments and I'm just really afraid that one day something might happen to her. 
Faith specific to the future was less overtly expressed than anticipated. Maria, the mother of a 14-year-old daughter, was very quick to respond:

I believe in God, and I believe she's going to leave this world whenever God wants her to leave,... and I'm not in denial at all I just don't believe you know that science can give me all the answers. And so I make plans like my other children, so she can start college when she's 16 .

Within the broader interview, distressed feelings were voiced, "...creates $[\mathrm{CF}]$ difficulty knowing there is no cure." With the median predicted survival age into the 4th decade, greater optimism is emphasized while hope for a cure continues to loom large in the $\mathrm{CF}$ community. This optimism, often guarded by some mothers, is projected, yet reinforced by health care providers. Pulmonary measures of lung health are evaluated quarterly, providing an indication of severity over time. Severity of CF has been reported as independent of maternal well-being or hopefulness factors (Cousino, 2013; Foster, Byron, \& Eiser, 1998). Therefore, mothers talk of the future with hope intertwined with guarded optimism.

It's just hard. There is no way to contextualize the multiple descriptors attached to what was described as hard shared from maternal voices; the message was CF care is hard. This word was used 169 times, textured within various contexts. All of the mothers described the beginning, either learning of the $\mathrm{CF}$ diagnosis or the required care, as hard. mothers gave perspective to hard. Lucia, the mother of three sons with $\mathrm{CF}$, stated:

I think it's hard, my life is hard I don't think people want it.... I mean, I know nobody would want my life, nobody would want to be in my shoes but since I 
have to wear them it's like I'm just gonna go with it, and keep on, keep on running, keep on trucking.

Treatments and medications are intrusive reminders of having a child with a chronic condition, a child "other than normal." At the present time, individuals with CF must do time-consuming treatments multiple times each day throughout their life. Maria, whose daughter is 14 years old, said, "It's really hard, It's really hard because all the medications, the treatments and you're seeing your daughter that she wants to be normal like everyone else and you know it's, hard for her, so that is hard [mother's emphasis] for me.".

Debora, the mother of two sons, said, "The hardest thing for me is the treatments, seeing them like having to do that so constantly and then knowing that they're going to have to do that their whole life".

Ana, who has a 14-year-old daughter, said, "The hardest part of everything has been probably understanding why. I question myself many times: Why did this have to happen to me? Why?"

Sophia, the mother of a 9-year-old son, stated:

I have to break into two pieces being the support and then, well, yeah it's just it's hard and like I know I have the support from my whole family but sometimes I do I do question like well, why me or where does it come yeah I just question myself. In the preceding segment, the metaphor of breaking in two, aptly described the conflict mother's experience trying to fit time-consuming CF care into her day-to-day life. In this moment, she talked about the challenges when her son was hospitalized, juggling demands of schedules with after school care and general care for her younger 
two children. While the mantra of the family is the patient is often spoken in pediatrics, the reality is that the mother or father must meet care demands of whole family. CF is widely described in the literature as having a high burden of care (Bluebond-Langer, 1996; Carpenter \& Narsavage, 2004; Christian \& D’Auria, 1997; George et al., 2010; Gjengedal et al., 2003; Jessup \& Parkinson, 2010; M. McCubbin et al., 1984; Moola, 2012; Szyndler et al., 2005; Tracy, 1997; Williams et al., 2009). The shared voices from these mothers are consistent with studies from the dominant Euro-American lens of mothers. While all 10 of the mother's described their work as hard, one mother added, "I feel that this is a very difficult homework or assignment" and a second mother of 17year-old daughter stated:

I just consider it an extra job or just another duty that I had to do... when we go in to appointments if the pulmonary function tests come back like $70 \%, \ldots$ and I already start thinking..."this is not looking very good" and...I automatically think treatments,... and so we do every 3-hour treatments. So the next week when we one time we were at $90 \%$ so it was quite an eye-opener for me, like wow like that has been all of my work.

In the account above, this mother not only viewed her care of her daughter as an extra job, she measured the success of her mother work by the results of her daughter's pulmonary function test results, reflective of her practice of mothering.

Acknowledging that their work of mothering has been hard from the onset, mothers openly shared their perspectives on subjective ways they manage. Lucia, the mother of three sons with $\mathrm{CF}$, stated: 
I just hope for the best and expect if the worst happens, it happens and we deal with it when we get there. I don't spend a lot of time obsessing or worrying over every little thing...I think it disrupts what your original plan was; you think you're going to have these kids, everything's going to be great, you're gonna have, you know, some little struggles here and there but then they're gonna get older and you start thinking about you know they're gonna have kids and you're gonna you know be an older adult and just enjoy your grandkids and things like that and that all gets kind of blurred because you really don't know. Not that those are guarantees before you knew that they had CF, but we like to think that they are.

Marta, the mother of six sons with CF, said, "I mean I can't sit and get depressed over it or sit there and ask why, why, why? I'm not gonna get the answers. It is what it is and have to move forward with them.

Sophia, the mother of a 9-year-old son, said, “... and then keep on moving and keep on being there for him being the support and then, well, yeah it's just its hard." While faith was not overtly talked about concerning mothers' views of the future, it emerged throughout the interviews as a source of support and strength. Marta stated: I pray every night just to have that patience for them and to help them out. And it's helped out a lot, it helps a lot.... Life is short even if you're not sick things can happen so that's my way of getting over this.

Estela, the mother of a 14-year-old daughter, said, "It's been hard. I've been asking God to give me the strength I need to get through every day." 
Alba, the mother or a 4-year-old daughter, said "I know that God left her in my life for a reason."

Gloria, the mother of a14-year-old daughter, said, " ...when we're in the hospital that God, in some way, is part of it.... That the doctors and nurses are doing God's work and helping my daughter and I feel all the problems are solved."

Teresa, the mother of an 8-year-old daughter, said, "...in God, [is] just the only thing we can get support from. With faith, just we don't see, we just trust, that's faith."

Religion and faith have traditionally played a strong role in Hispanic cultures. The "Virgin of Guadalupe, the patron saint of Mexico and Central America" (Sandoval, 2008, p. 13). is worshiped devoutly throughout Mexico, symbolizing the good mother. The mothering characteristics transferred from this symbol link to marianismo, selfsacrificing and highly protective mothers (Chang \& Liou, 2009; Dreby, 2006). In Latino gender ideology, ideal mothers are described as doing their greatest activity as primarily caring for children. They are characterized as self-sacrificing mothers, having the highest calling and judged in terms of their morality. The influence of this ideology might be also applied to understanding the profound way mothers described calling on their faith as a guiding force in their daily lives.

Ascribing agency to God through doctors and nurses is consistent with empiric studies on faith and Hispanic families of chronically ill children. Fatalism, also culturally described as a world view associated with faith, did not emerge (Rehm, 1999). Having a child with CF inspired a higher purpose or life's meaning for both Gloria and Alba. Parents try to make sense out of their life stories. Attributing meaning from faith is one 
way a sense of comfort is derived from the day-to-day work of mothering (Gallagher et al., 2008; Pizzignacco, Mello, \& Lima, 2011).

Being here emerged as a major theme with four subthemes: (a) parental gendered differences, (b) more mother talk, (c) education, and (d) communication won't happen without an interpreter. The theme of being here evoked the mindfulness demanded of mothers within each subtheme to determine at each moment what strategy is best for her child, her, and the collectively as a family. Each area carried a tension that mothers navigated to maintain relationships, some with a greater degree of power dynamics than others.

Being here. Parental gendered differences. "Well I think parents from anywhere, we just try to give her best samples [examples] for the things for our kids." Mothers voiced their impressions of cultural influences on parenting. The above segment, voiced from a mother (of her 8-year-old daughter), encapsulated much of the underpinnings of mothers' talks, yet other topics stood out. Three mothers expressed concerns about gendered differences with their spouses. They expressed the difficulty their husbands have had accepting the diagnosis. "He still sometimes doesn't accept the fact that they [two sons] have CF." One mother suggested her husband was influenced by the culturally associated "macho male" identity, "For my husband, I noticed he doesn't like to talk about it $[\mathrm{CF}]$ in front of the male family because they make it seem like you produce weak offspring," While one mother stated her husband does not want her to talk about their son's CF, “...like my husband, he's more like he doesn't like to talk about what he has or the CF and then I'm more open to [than] that." While mothers expressed concerns about their husband's acceptance of their child's CF diagnosis, they did not expose 
specific marital discord. Gendered health beliefs and behaviors are a product of cultural values, norms, and identity intertwined with role socialization (Addis \& Mahalik, 2003. A father's emotional response to his child's CF is complex, and in part, is subject to his masculine identity and perhaps his own pattern of health seeking experiences. Hayes and Savage's (2008) qualitative study on the influence of CF on fathers' management confirmed that emotional withdrawal was one mechanism used to manage their situation yet occurred simultaneous with emotional involvement. One dad emphasized that fathers are not asked about how they manage, indicating feelings of marginalization. The traditional Hispanic, macho male rooted in Latino culture provokes images of threatened masculinity as one speculation for emotional withdrawal. While fathers might contextualize cultural and gendered behaviors, it calls to mind the potential influence of posttraumatic stress symptoms discussed earlier.

More mother talk. One mother identified, "I think like culturally the whole doctor, going to the doctor is more of a reactive thing and not a preventative thing." Mothers' expressed curious experiences with their extended families about CF treatment. The notion of "your body gets used to it" was put forth in three interviews related to medications. Their extended family members questioned why the child with CF took so many medications. One of the mother's beliefs mirrored this similar mind-set. Bearison et al. (2002) described similar maternal comments from his study on beliefs and use of folk medicine for children with asthma. A contextualized explanation was not offered. However, there was an attempt to link the aforementioned comments to the Hippocratic theory of disease and the four body humors. In the Hippocrates theory, the wet-dry and cold-hot approach to balance health and illness is practiced (Spector, 2004). This view 
was expressed by one mother, "...trying to prove to the doctor that the weather could possibly have something to do with it" (her daughter's recurrent CF illness, prior to her $\mathrm{CF}$ diagnosis). Three plausible explanations are offered: confusion of $\mathrm{CF}$ treatments is associated with the development or potential for antibiotic resistance; a lack of clarity exists when the progression CF necessitates a step-up approach for medication management; controlled symptoms do not equal improved health. Mothers talked about what was said by their family in the following passages:

"Oh, that medication's not gonna work in 10 years because the body's getting used to it."

"Do the natural way it's gonna kill them sooner if you keep giving them this medication."

"You know, her body to get accustomed to the certain medication even though they are natural, but still you know she's still going to get accustomed."

In addition, extended family members' attempted to deter medication administration based on the child's physical appearance is concerning:

"Oh, you know what I mean. I see him and he's well with the medicine. So there's no reason to do something extra for him."

"...because a lot of them look at her and think she has nothing."

"Why are you giving your child medicine if they seem fine right now?" Feedback from extended family of this nature is difficult for a mother to counter, as one mother stated:

You're going against everything you were kind of taught... because you have to go against all that and you learn to be a little more proactive even though 
everyone in your family thinks you're crazy and downright thinks that's wrong for subjecting your child to all that.

This discourse presents a constellation of concerns particularly in the context of family values, familismo. Speculation for such discrepancy stimulated consideration toward a differentiated level of acculturation or generational influences. Last, the historically infrequent (or under diagnosis) of CF in the Hispanic population could preclude a family member from recognizing the magnitude and implications of stopping CF medications.

Education. Mothers were quick to identify education as a culturally influential factor in their parenting. One mother of a 14-year-old daughter stated:

Okay, I think it has to do with education, I think it's a big big, they key is education because when Bianca was diagnosed with the $\mathrm{CF}$, even though I went with a genetic counsel, and I think I visit her like three times and I didn't get it. One mother of a 9-year-old son said, "....also very important for families to feel comfortable asking questions, that's also very important and a very important way to find and get important information and have more education to ask questions."

A mother of two sons, 5 and 7 years, stated:

Well, just knowing and being educated sometimes helps and that's why I agreed to participate in this study because I believe it could be beneficial for families in the future with the care of other patients with CF. So I feel like having information and just kind of learning what it is and what I might expect coming up in the future would be helpful. 
A mother of 14-year-old daughter stated:

Just sometimes not having the experience, but I would have like or would like to share my experience or see what other Hispanic mothers have experienced to kinda see what has worked for someone else and maybe that could help me or I can help someone else with my experience. Also with the stage of my daughter is in that's definitely an area where I could have a little more help, helping them better understand their illness and better understanding [how] they [mothers emphasis] feel and how they view themselves like in comparison to other healthy peers.

Education has been identified as highly valued across Latino national origins rooted in collectivism, or interdependence, and underpins parental decision making (Halgunseth, Ipsa, \& Rudy, 2006). In their comprehensive literature review on parent control, Halgunseth et al. described education as a goal of childrearing, training in morality, responsibility, and interpersonal relationships. These training practices intersect with the practice of mothering Ruddick (1995) described and underscore the mothers' perspective. In addition, the mothers expressed a desire to reach out to increase $\mathrm{CF}$ awareness and to learn from each other. Relationship affiliations Gilligan (1993) assert that women gain a sense of self through connection. Mothers expressed a desire to reach out and connect with other mothers either going through similar parenting challenges or reaching out to mothers of newly diagnosed infants. Development of interpersonal relationships to receive individual support or provide support to a newcomer in the $\mathrm{CF}$ community suggests that for mothers struggling to find balance in their $\mathrm{CF}$ individual world, they desire to connect with mothers similarly situated. Their interest to increase 
awareness about CF highlights their spirit of interdependence locating them within their cultural landscape.

Education for mothers was valued to keep informed as many stated. Mothers confirmed the CF center as their primary source of information and updates on CF. Use of the Internet as an information source was limited; three mothers specifically mentioned they were cautioned not to rely on the Internet for accurate $\mathrm{CF}$ information. All mothers were queried about what they understood to be the cause of $\mathrm{CF}$, its treatment, and cure as a means to validate their understanding of CF. Nine of the 10 mothers responded with fairly brief descriptions such as its genetic origin. Most mothers filled in a little more details of phenotypic characteristics in satisfactory terms; one gave a detailed description of the protein defect. One mother unexpectedly responded, "It's something that I guess you can't avoid. I guess I'm not too much informed or really haven't paid attention. I know it's there and I just have to deal with it." Another mother said, "That's what I don't understand like the cause, nope..." and continued to talk about what treats CF. In the former account, there were no other indicators that raised doubt about her mothering related to CF care. In the later situation, although an interpreter was declined, it is possible that limited English proficiency confounded her understanding of the medical details involved with CF. Medical information often needs simplification for Englishspeaking natives; I believe this mother will benefit with an interpreter in the future.

In the interest of exploring maternal health beliefs, mothers provided information on their use of remedies garnered from Hispanic traditional home beliefs. The intent was not to conduct a full assessment of complementary and alternative therapy. Likewise, some of the mothers did describe familiar practices such as amulets, healers, special 
potions, and such that their extended family had encouraged them to try. Table 10 summarizes responses for both pre- and postdiagnosis. Reported practices were similar to literature (Tanase \& Zanni, 2008). Most commonly reported agents were lemon, honey, and cinnamon teas. Chicken fat was recommended by one child's grandmother to counter phlegm. In another account, a sister-in-law (who also has a child with CF) recommended to stop oregano to avoid interfering with prescribed $\mathrm{CF}$ medications. Oregano and olive oil are reported to have a wide range of indications, such as effects as an antimicrobial for Pseudomonas aeruginosa and as an anti-inflammatory agent respectively (Thomson, 2004). Neem was given for its properties to inhibit aflatoxin by aspergillus organisms (Costa, Geraldo, Arroteia, \& Kemmelmeier, 2010). More alternative therapies were tried prior to diagnosis than following diagnosis.

Table 10

Use of Home Remedies

\begin{tabular}{|c|c|c|}
\hline ID \# & Before diagnosis & After diagnosis \\
\hline M1 & prayer for healing & prescribed CF therapy only \\
\hline \multirow{2}{*}{ M2 } & meconium ileus, & "juices" for health; neem, oregano oil, olive \\
\hline & diagnosed at birth & leaf; yoga \\
\hline \multirow{2}{*}{ M3 } & & prescribed CF therapy only; \\
\hline & & learning meditation \\
\hline \multirow[t]{2}{*}{ M4 } & diagnosed at 2 weeks of & fried chicken fat; omega-3 \\
\hline & age, none reported & \\
\hline
\end{tabular}




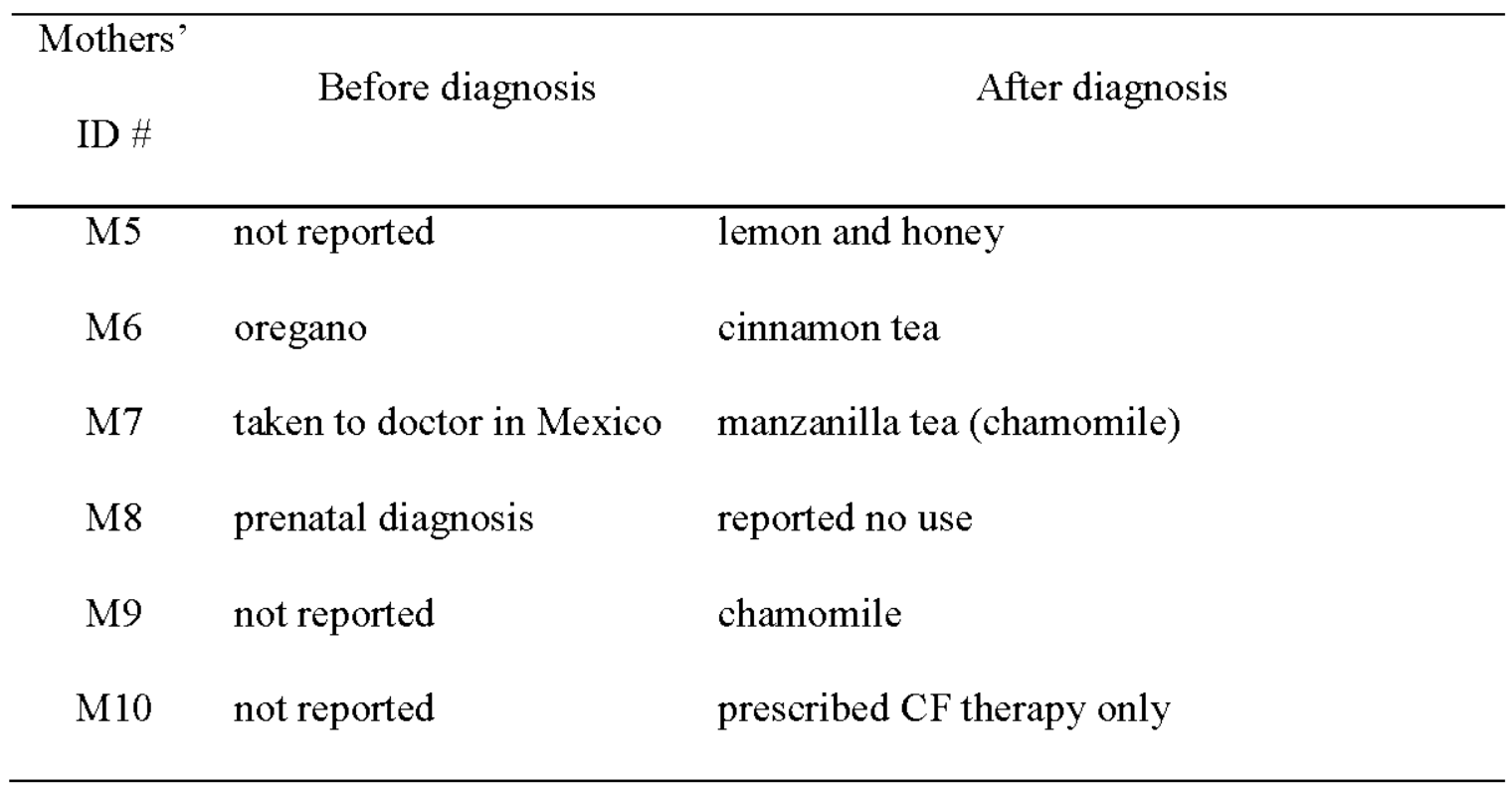

Communication won't happen without interpreters. Mothers were thoughtful in responding to the interview inquiry: Some families from different ethnicities tell us they have experienced disrespect or discrimination with their health care, I wonder what your experience has been? This query elicited a range of responses. Four mothers denied having such experiences.

Gloria (M6), the mother of a 17-year-old daughter, said, "I don’t ever remember feeling discriminated or being treated differently. They've always been very helpful." Debora (M8), a mother of two sons, Mario, 7 and Rafel, 5 years old, said, "From my experience, I have not felt any discrimination, going and visiting at the hospital I have felt welcome and almost as I was at home."

The above two mothers were quick to deny any uncomfortable experiences. While the following two mothers stated they had not experienced disrespect or discrimination, they related experiences indicative of power relations: 
Sophia (M7), mother of Luis, 9 years, stated:

Well, no it hasn't happened to me. I guess, I guess like being Hispanic then I guess it doesn't make any difference, but as Hispanic we don't have that much like information or I don't see it as much....We don't ask for those questions, like, we just we just umm get the information, get the medicines, we have to take for him and then that's it.

Ana (M9), mother of Isabel, 14 years old, had the initial response, "I don't feel

like I ever had an experience where I felt discriminated or that Isabel has been

discriminated. I feel that we've been treated equal to all of other patients and don't think they treat us any different."

As an example of power relations, Ana stated:

In the beginning, a lot of doctors would offer [an interpreter] and ask if I needed an interpreter and it was available. However, but now not everybody will ask or offer that. They sometimes will have Isabel kind of as a translator. That's still very difficult because sometimes there's terms that she doesn't even understand and maybe can't explain it to me. Sometimes I have questions and I have Isabel kind of ask questions, and hopefully she will understand, but even then she will tell me that she doesn't even understand what they just told her.

Ana went on to describe how the CF team can help take care of Hispanic families better, "...because maybe the doctors seems in a hurry or I know there's a lot of patients to see. Maybe the moms don't feel comfortable asking for it [interpreter] but if it's offered it would definitely be more helpful." 
Sophia (M7) described having limited access to CF information. Her comment suggested that she doesn't ask questions either from lack of opportunity or not knowing what to ask. The issue of physician time has been cited as a barrier superimposed over the cultural value of excessive respeto for physicians that prevents asking questions (Mayo, Sherrill, Sundareswaran, \& Crew, 2007).

From a critical feminist lens, in the above case, Ana (M9) might not understand not having an interpreter marginalizes her maternal role, hinders her understanding of information, and prevents interactions with health care providers for an accurate exchange of information to the ultimate end of taking care of her daughter. This gap serves to undermine maternal self-efficacy that can lead to self-doubt and potentially robs her ability to provide care, training, and nurturance within her maternal capability. While health care struggles to meet the needs of diverse populations and cost containment, it perpetuates a patriarchal system that continues to tolerate control of information and interactions. This, in turn, limits active participation, as a parental right, reinforced by limited access to interpreters.

Language and communication styles have been widely identified as barriers and dissatisfiers contributing to health disparity (Mayo et al., 2007; Ngui \& Flores, 2006; Smedley et al., 2002b). They also have been reported as key determinants of dissatisfaction for Spanish-speaking Hispanic families with children with special health needs as well as for families with limited English proficiency (Ngui \& Flores, 2006). Information exchange related to limited English proficiency constitutes an additional factor setting the stage for restricted communication. These factors affect patient outcomes and quality by deterring active engagement and partnering between mothers 
and providers. They might lead to health care providers assigning binary labels of families as adherent-nonadherent and place blame on mothers rather than the organization's lack of prioritizing the importance to increase resources, such as interpreters, or hold staff accountable to utilize existing available resources to support quality care and reduce disparities.

Health care systems can creatively implement different delivery care models for underserved and marginalized populations. Minors used for interpretation to convey medical information is inconsistent with Prohibition against National Origin Discrimination Affecting Limited English Proficiency Persons (Executive order No. $13166,2000)$. This regulation states that reasonable steps must be taken to provide limited English proficient individuals with meaningful communication and appropriate allocation of resources. Reasonable is the operational term that needs clarification.

Two mothers affirmed experiences of perceived discrimination. One clearly responded with yes and one framed her experience as culturally insensitive. Maria (M2), who has a 14-year-old daughter, said, "I would have to say yes" when asked about her experiences of feeling disrespect or discrimination. She added:

When I had to ask the nurse in regards to contact the physician and I feel that when, I would say it's not my opinion, but in a way it is, I feel when they see a Latina person,... and more with me that I have my accent, and it's really heavy accent, they think you're not educated [her emphasis].

Although this study did not inquire specifically about generations, years living in the U.S. did come up in some of the responses. Lucia (M1), a second-generation English- 
speaking mother of three sons with CF expressed the following when queried about experiences of disrespect or discrimination, ...not super discrimination but not so much understanding. I've had issues with the dietitian as far as foods that we eat and stuff, basically like being told to stay away from beans and tortillas things like that because those are kind of staples like eggs and milk....maybe certain things aren't understood and yes, food was one of them. I thought that was a little bit insensitive... so being told to maybe stay away from those things, it's kind of like not as sensitive as you know it's kind of like ripping off an arm. I know it's not necessarily good for you, I get that, but its comfort food, it's just what you eat all the time.

Lucia continued to describe additional issues of concern:

As far as discrimination, not too much, as far as getting care. I think we're in California too, so I think it helps ya know you see other patients, ya know it's not like we're in Minnesota or something. I would think that would be harder. The only thing is just the whole assuming our kids didn't have it [CF] because of their ethnicity and being told we were being denied diagnosis from our health care plan [obtaining authorization for out of network consultation at a $\mathrm{CF}$ center]...we have a team, three little fighters, and we've participated in that [fund-raising walks]...for my kids, too, they don't really see other kids that look like them that are featured in the advertisements or the pamphlets.... I think if people would stop saying it's a Caucasian disease... if they showed people with other faces that have it...the kids' pediatrician... after she found out that the boys were diagnosed...but 
she said you know, she says, they have had asthma or bronchitis or whatever, but I never thought to test them.

Lucia's (M1) three little fighters fund-raising team title, metaphorically mirrors her ruptured relations with her early health care experiences, situated in her perceived marginalized ethnic position: the pediatrician failed to consider $\mathrm{CF}$ as a diagnosis for her children, her kids don't see other kids who look like them in CF marketing brochures, reference to CF as a "Caucasian disease," and the pediatrician stating "she never thought to test them." Her team of three little fighters symbolizes the family's motivation to create more awareness of CF beyond Caucasians within their socially situated world. Cultural insensitivity was described as "not so discriminatory" as she proceeded to describe her experience metaphorically "ripping off an arm." This graphic depiction moves perception beyond cultural insensitivity with vivid details that incite feelings of pain, disconnection, and disembodiment. The use of metaphor was employed; it is a common literary device used to express emotion (Riessman, 1990). In this moment, this mother provided a sense, perhaps unconsciously, of feelings distanced from conflicted emotion. In addition, Lucia's metaphor further suggested a perception that relinquishing her cultural foods severs, and borrowing Ruddick's (1995) terminology, her innate maternal capacity to nurture her sons.

Some mothers acknowledged the infrequent occurrence of CF in the Hispanic population. Estela, mother of Natalia, born in Central America, now 14 years old, said, "I don't think before they used to diagnose Hispanics with that diagnosis because it was thought that most of the Caucasians population would get diagnosed." Debora, mother of two sons, 5 and 7 years old, said, "I definitely feel that CF is still very new to the 
Hispanic population so a lot of them don't know about it." and later shared she would like to increase awareness of CF in her Hispanic community.

In summary, being here is conceptualized in several contexts. First, it represents the potential for marital conflict in a traditional patriarchal family system. Emotional withdrawal, versus being present, provoked marital tensions when support and communication were needed in the context of family function. A mothers' presence is the "emotional heart of the family" (Dreby, 2006, p. 36).

Second, the practice of mothering perceived to be in resistance to extended familial values and beliefs provoked tensions within the extended family system, and is of concern. In the traditional family setting, as one mother related, adherence to the prescribed medical care in the face of unsupportive extended family fractured them because of this conflict. Countering extended family's health beliefs and values created a familial split, resulting in isolation and lack of much-needed familial support systems.

Last, being here relates to accounts from mothers' situated with their child in health care encounters, for the most part, and did not voice perceived experiences of disrespect or discrimination. This response is not surprising since mothers, also recipients of their child's care, are in vulnerable positions. They might wish to relate their experience of being treated different, as some have, yet they worry that a complaint or account of an inappropriate event will put their child's care in jeopardy. Mothers might decide to, so to speak, be here with their experiences, acting to protect their child the best they can. Decision points assert a mothers' way of knowing, through her experiences, she has learned to trust herself, to be quiet, to protect, as in subjective knowing defined by Belenky et al. (1997). In contrast, a mother might find herself subordinated by and from 
the imposed hierarchy, described as received knowing in Belenky's et al. typology of women's ways of knowing. "Received knowers subordinate to authority" (p. 15). Vulnerability within the hierarchical health care environment is not unique to Hispanic or Spanish-speaking mothers. However, when an additional barrier of language or cultural difference exists, vulnerable feelings and the imperative to protect might be intensified. Power relations might be subtle, less tangible, yet concerns mount for such possibilities where empowerment and patient and family-centered care are promoted inclusive of cultural diversity.

This study elucidates the value of the practice of mothering, a framework Ruddick (1995) initially proposed to give meaning to mother's work. Applying this framework to Hispanic mothers co-constructs their profound commitment to the care of their child with CF. Application of maternal thinking intertwines the primary elements of mothering, preservative love, nurturance, and training in a sociocultural context as it relates to mothering a child with CF. In this study, the practice of mothering from the Hispanic landscape resonated with culturally defined goals of childrearing described in Hispanic families (Halgunseth et al., 2006).

In addition, not unexpectedly, mothers described a child-centric life with requirements of $\mathrm{CF}$ care demands consistent with existing literature, albeit from dominant Euro-American studies. Life disruptions were expressed around unknown manifestations of $\mathrm{CF}$ as mothers moved through descriptions of their daily lives, cautiously planning futures. Family tensions resulted when nonconformity of cultural beliefs collided with family values. While the majority of mothers denied feeling marginalized in their health care experiences, four mothers identified cultural insensitivity or a failure in the system to 
support equal care as it related to those who require interpreters. Therefore, mothers were deprived of their full-embodied mothering practices. Mother's expressed gratitude for their child's health yet their denial of disparate treatment becomes a paradox that merits attention. Such familiar experiences become internalized and become routine. A counterpoint to this perceived disparity is the enduring relationships established over time through repetitive medical encounters with $\mathrm{CF}$ health team members. However, opportunities exist for culturally appropriate behaviors such as personalismo. Enduring health care relationships do not excuse disparity.

Health care environments and nurses must embrace diversity, support mothers, and support mothers in finding their voice. We must make visible the value of the practice of mothering toward empowerment. Through the complex layers of valuing and communicating, the action of diminishing disparities becomes possible. 


\section{Chapter 5: Discussion}

This study explored the perspectives of Hispanic mothers' experiences parenting their child with $\mathrm{CF}$ through narrative inquiry. Both structural and thematic analysis illuminated mothers' experiences. To my knowledge, this is the first study to represent Hispanic maternal perceptions of caring for their child with CF in the U.S. One Brazilian abstract (Tavares et al., 2010) had a similar purpose; findings portrayed mothers' lives shrouded in sorrow, anguish, doubt, suffering, fear, and reorganization of family. A more recent ethnographic case study of three Brazilian families (Pizzignacco et al., 2011) sought to understand dimensions of CF experiences within the family context. Life expectancy in Brazil was reported to be" around 18 years" (p. 637), in part, because of delayed diagnosis. Thus, maternal depression depicted in the former study, might be explained by the shortened life expectancy in developing worlds with limited health care resources.

Mothering in this study represented a major theme in addition to growing and growth, mother talk, and connected, evoked from the profound language Hispanic mothers voiced. Subthemes that emerged were life disrupted and being here. The study was sensitized from a critical and feminist lens, elaborated in the discussion. Ruddick's (1995) framework further informed the analysis.

Mothering and its elements of practice are threaded throughout the literature as components of what mothers do. Although discussed extensively throughout the literature, locating its fundamental imperatives often requires reading in between the 
lines. On closer examination, mothering practice's taken-for-granted elements are evident related to children with and without health problems, and are also found within the Latino cultural mothering contexts (Chang \& Liou, 2009; Halgunseth et al., 2006; Hughes et al., 2008; Rueda et al., 2005). These studies reflect foundational mothering. Specific elements of mothering are evident: preservation and love (Gallagher et al., 2008; Glasscoe \& Smith, 2011; Hodgkinson \& Lester 2002), nurturance (Cronin, 2004; Gallagher et al., 2008), and training (Cronin, 2004; Strom et al., 2008). In addition, mothering elements within a larger sphere of maternal roles have been described (Berge \& Patterson, 2004; Chang \& Liou, 2009; J. S. Coffey, 2006; Halgunseth et al., 2006; Sullivan-Bolyai et al., 2004).

Overall, maternal narratives unfold a story of mothering through their constructed social and cultural realities that give meaning to their daily lives through the practice of mothering. The construction of mothering and caring for their child with CF was informed by Ruddick's (1995) framework. The findings from this study are consistent with descriptions of mothering and provide a background for understanding the maternal role. A distinction in Hispanic mothers' roles is their dedicated maternal calling inspired from the Virgin of Guadalupe. Although Ruddick's framework casts a wide net of applicability to mothering, "it has been underutilized by those who could benefit most from their use" (Radosh, 2008, p. 305). Applying this framework, calls forth the so-called elusive work of mothering to one of more conscious solidarity to augment its meaning and value of the work mothers do.

Ruddick's (1995) work has not been without criticism. Feminist scholars have attacked it for perceived attempts to apply it to the universe of mothers developed from a 
narrow socially constructed world (Bailey, 1994; Collins, 2007; Glenn, 1994). Ruddick (2007) addressed these criticisms, defining her work as contextualized from a "middleclass, white, Protestant, capitalist, patriarchal America" (p. 97) constructed world. She asserted that her work does not govern all mothers, but that parts of her work have universality. I would argue that the central themes presented here fit the universality of mothers. Mothering in Ruddick's framework is not without flaws, just as children are not all from the same mold. Some mothers are not able to mother for a variety of causes. She asserts mothering to be the authentic work of mothers.

Through a critical feminist lens, the first aim of this study was met by opening a space for Hispanic mothers of children with CF to uncover what has not been told or has been ignored and is now reflected in their embodied stories. According to Devault (1996) a commitment to finding women and uncovering their concerns and supporting changes that will improve women's status constitutes a feminist approach.

Ruddick's (1995) work fits with this study in that mothers embody the essential elements inherent in its practice. The mothers expressed a strong focus, across all children's ages, for preparing and educating their children within their capacity for future social acceptability. The study affirms the concept analysis of parenting where the authors found three critical attributes: The mothers certainly protect their children, being there for them to supervise, support, or guide. They provided evidence of nurturance. The work of mothering shows commitment to advance their children, encouraging opportunities not afforded by them, as parents. They are motivated by their children, for their children, and with goals to see them positioned as socially acceptable. 
Mothers were questioned about their recall of participating in genetic counseling, a standard CF clinical practice. Once an infant or child is diagnosed with CF, all siblings are tested because of its genetic transmission. An unexpected discovery in this study was four mothers had no recollection of genetic counseling. Furthermore, all were first-time mothers: three had subsequent children, one mother was with her same spouse, two were with different spouses, and one mother remained separated from the biological father since early in her pregnancy. It is unclear from these four mothers whether genetic counseling was offered. Genetic counseling is practiced as a component of CF newborn screening programs, inclusive of all 50 U.S. states, at one end of the spectrum (The American College of Obstetricians and Gynecologists, 2011). At the other end is the narrative of reproductive freedom. Stereotypes of Latina women have provoked a struggle to shed gendered myths and misconceptions (Gutierrez, 2007). In contrast to Latina stereotypes of fertility, passivity, and Catholicism, Gutierrez writes about prochoice Latina women who have fought hard to win reproductive freedom. While there might be growing efforts to promote reproductive freedom, its views are diversified, embedded in sociocultural values. Although prenatal testing is a reproductive option, access and implementation of these services might elude underserved women. It is not the intent here to promote or defend family planning, a topic beyond the scope of this study. Its intent is to promote reproductive freedom for informed decision making for women. Reproductive freedom was called to action from the first wave of the feminist movement. When women are uninformed about the prenatal testing, they are unable to move away from gendered oppression to exercise individual choice and decision making. 


\section{Cultural Influences on Hispanic Maternal Parenting}

Three salient concerns were uncovered around influential cultural factors mothers voiced on their parenting. These factors included education, gendered spousal concerns, and influences of extended family members.

Education was stressed as one influential cultural factor related to parenting. A recent literature review on Hispanic parenting highlighted education (Halgunseth et al., 2006) as a critical goal that directs decision making across Latino regions; and as a purposeful parenting activity (Virasuru, et al., 2011). Mothers valued being updated about $\mathrm{CF}$ primarily through the $\mathrm{CF}$ center. Mothers voiced the value of training and supervising by way of mother talk as well as their recognition for additional support or assistance with parenting, for example, "also with the stage...that's definitely an area where I could have a little more help, helping them better understand their illness." In addition, collectivism was represented in their expressed desire to reach out into their Hispanic community to bring an awareness of CF, "I feel like I would be able to help...other families, maybe through groups or counseling... so I would like to be able to do that." Through a feminist lens where women historically have been oppressed, taking an active role in their health is political in nature by promoting awareness and improving their circumstances. Mothers are positioned to take charge and fully participate as the primary actors of their child's care, extending their cultural voice. In this way, a start to transform one individual at a time toward a group consciousness unfolds (Campbell \& Bunting, 1991).

Mothers voiced concerns about the emotional distance spouses placed between the issue of $\mathrm{CF}$ and their child. Interestingly, the three mothers who expressed worries all 
had sons with CF. Directly interviewing their gendered counterparts might shift the perspective to different ways of understanding. It is not clear whether the mothers' observations reflected a traditional gendered masculine view; in other words, would daughters with $\mathrm{CF}$ motivate similar paternal concerns.

Hayes and Savage (2008) quoted a father's comments, "I've no problem talking about CF, I've no problem talking about my son, but I have a problem talking about my son with CF"' (p. 253).

Likewise, one mother shared an experience with her spouse, "like when they [multiple sons with CF] finally they lost their pancreas sufficiency...He [father] was devastated. In his mind, he just thought they were just going to shrivel up." Often loss regarding $\mathrm{CF}$ is talked about in the context of quality of life or end of life, commonly from respiratory failure. In this moment for this father, the loss of pancreatic function was a loss resulting in malabsorption requiring added treatments, adding to the burden of care. Fears of future loss of organ function, such as the complication of CF-related diabetes might also be perceived as an incomplete loss. There are many potential physical losses a person with $\mathrm{CF}$ might experience that alter quality of life; loss or diminished smell from sinus complications or loss of body image as their body becomes marked with devices to augment care, such as gastrostomy tubes and vascular access devices. Listening to mothers (and fathers) describe their experiences opens new ways for health care providers to support, offer, and intervene through empowering parents in decision making and coping.

These accounts call to mind consideration of ambiguous loss theory (Boss \& Carnes, 2012). This theory is concerned with incomplete or uncertain loss as a result of 
the disappearance of body or mind that manifests in ambiguity. Energy is mobilized to reconstruct meaning and hope. Parents and individuals might experience $\mathrm{CF}$ through a process of partial or incomplete loss (Boss \& Couden, 2002). They are physically present, but not fully embodied with full physiologic function; uncertainty of further loss with other bodily functions might emerge in unpredictable patterns. The incongruence of how well a child with CF might appear from their physical appearance is deceptive to outsiders who do not understand the damaging internal effects "... because a lot of them look at her and think she has nothing." Boss's theory might also assist families in making meaning and give perspective to such differences. Several mothers gave similar accounts.

The application of Boss's theory is non-gendered, pertaining to fathers and mothers. Although the mother's quote below articulated the genetics of $\mathrm{CF}$, existential questions exist, "Sometimes I do I do question... why me or where does it come, I just question myself." Self-doubt creeps in when there is no perceived rationale or context for understanding, when science just might not suffice. Loss perceived through genetically transmitted conditions is yet an additional life circumstance to explore through this framework. Therefore, exploring $\mathrm{CF}$ through an ambiguous loss lens might illuminate a less pathological association of grief and sorrow toward new insights for reconstructing life's meaning.

Findings related to disrespect and discrimination were consistent with current studies exploring similar topics. The majority of mothers denied experiences of perceived disrespect or discrimination. Several mothers recalled events implicating power differences. Language is consistently cited in studies, and was in this study, as problematic even with interpreter support (Bonilla \& Edwards, 2011; Gonzalez-Espada, 
Ibarra, Ochoa, \& Vargas, 2006; Hughes et al., 2008; Mayo et al., 2007). Hispanic mothers, disadvantaged by language, cannot transform into responsible, active caregivers when medical encounters not conducted in their preferred language continue to promote dependency and passivity through patriarchal dynamics (Freire, 1993).

Overall, mothers reported high satisfaction and deep gratitude regarding the health care of their child. The majority of mothers lauded the CF team, and in particular, praised the social worker for continual support and assistance. Spanish-speaking mothers expressed a desire for greater availability of interpreters along with printed Spanish and media resources, a continuation of language disparity.

Overall, the aims of this study were met. Through the mothers' willingness to share their stories, a deeper understanding of their situated experiences was uncovered. Their stories revealed concerns that stimulate further explorations in the areas of mothering, ambiguous loss, and power relations in the health care systems.

Health care systems must consider novel solutions to meet improved patient needs. For example, a split clinic day in tandem with scheduled interpreters reserved for Spanish-speaking families could better accommodate patient needs. This type of strategy could improve workflow while effectively managing resources. Increasing communication results in greater trust and in favorable outcomes for quality patient care. Empowering the family to perform its best care sends a message of personalismo, the valuing of human relationships (Chang \& Liou, 2009).

As health care systems advance, diversity must be examined by stronger efforts to meet needs. "To discourse on humanism and to negate people is a lie" (Freire, 1993, p. 91). In addition, adding a genetic counseling question to the routine medical history 
template throughout the 1st year post-CF diagnosis might open opportunities to identify disparate services that inhibit informed future family planning. In the midst of learning about a new diagnosis, genetics might easily confuse parents who are counseled during the immediate diagnosis period: "Even though I went with a genetic counsel, and I think I visit her like three times and I didn't get it [mother's emphasis]." Genetic counseling might need to be more individualized for greater effectiveness.

Cystic fibrosis health care providers routinely encourage families to treat children as normal and live as normal as possible. Two mothers specifically articulated normalization as a family goal. Normalization, defined by Deatrick, Knafl, and MurphyMoore (1999), is the subjective definition a family gives to its situation that shapes how its members manage it. This framework positions normal as the standard in health care. It has had limited application outside of the dominant Euro-American population. It presents a fertile area for future studies.

Many mothers might not identify with a self-view of feminism, never think of themselves from this perspective, but expect fairness; which is the most reduced form of feminism (Rothman, 1994). Although mothering might be thought of less as a feminist issue, it has everything to do with a political agenda. Rothman further recalled "motherhood is not only a physical and emotional relationship - it is also an intellectual activity" (p. 155). It is this "unity of reflection, judgment, and emotion," (Ruddick, 2007, p. 97). Ruddick called this "maternal thinking" (p. 97). Children are the future, and by embracing the foundation of mothering children, the future is embraced. Giving mothers hope concerning the future of CF stimulates them to do mother work and not dismiss their children because of a shortened life, as was seen in the not so distant past. 
Mothers whose experience is gendered, societal oppression constructed from role expectations must uncover ways their children can transcend marginalization through training and education (Radosh, 2008). Acknowledging the sociocultural barriers that impede and those factors that propel, the stage is set for mothers to be in an active role. The ability to reflect and critically think is essential for mothering; they are also essential to Freire's strategy, both working to transcend oppression in a patriarchal reality.

Education is a vehicle in both ideologies that is transformative toward more active roles for creating change in their sociocultural reality.

\section{Implications of Findings}

Implications for clinical practice and education. Nurses and health care

providers can provide peer and interprofessional support to strengthen cultural differences in practice. Supporting and reinforcing communication through the use of interpreters is essential to promote and optimize mothers' delivery of care. It models a critical necessity to narrow the gaps in disparate care. Nurses can listen to the voices of mothers, validate their mothering skills, encourage their growth in managing the care, and support caring for their child. Mothers indicated that education constituted a highly valued way of mothering. Education is at the heart of what nurses do to empower patients and mothers to navigate through their personal and public realities. Education can build toward empowerment. The process of empowerment occurs in a context with interactions with others, and with interconnectedness with the environment and the person (Gibson, 1995). Empowerment described by Gibson involved a "personal process where individuals developed and employed knowledge, competence, and confidence for making their voices heard" (p. 1201). A more common description of empowerment is the equal 
partnering of patients with health care providers in decision making for their health care (B. Patterson, 2001), and "to become active agents of their lives and well-being" (McLean, 1995, p. 1058). These definitions highlight empowerment from both private, in the former, and public, in the later, spaces. Studies on how to unite effectively disciplines to bridge empowerment from both private and public spaces are needed.

Future research. Ruddick's (1995) framework of mothering has broad implications for future studies applied to family nursing and mothering related to chronic conditions within sociocultural lives. Studies that continue to focus on mothering will highlight and support behaviors that encourage respect, value, and give credibility to mothers for their tireless caregiving. In addition, further exploration of ambiguous loss as it applies to $\mathrm{CF}$ and can augment insight into gendered behaviors yet to be uncovered. Exploration through the lens of ambiguous loss can also support and validate the individuals' experience and move them toward one of greater meaning making in their lives. Finally, exploring families with CF through the family management-style framework can further illuminate family function and how families manage life with a child with $\mathrm{CF}$ through a cultural lens. Focused studies on targeted ethnic groups will continue to uncover similarities and differences to direct individualized care.

\section{Limitations}

Narrative inquiry constitutes both a strength and limitation. The strength of this approach lies in its potential to understand more deeply the experiences, beliefs, and perspectives of Hispanic mothers parenting their child with CF. Study mothers comprised $50 \%$ of Spanish-speaking mothers, which brought me closer to the phenomena of interest to capture their untold voices. The Spanish interpreter was a bilingual child life specialist 
who interacts with children and families daily in a health care setting. Because storytelling was a focus of the study, my dissertation chair and I determined an interpreter with the aforementioned qualifications was adequate. This study is an initial step toward creating a path of advancing nursing science to increase the understanding of the dynamics of a Hispanic cultural group with CF. Voices of mothers form non-dominant groups are under- represented. It serves as an entry point to direct more culturally sensitive care approaches. The study did not attempt to stratify the health beliefs across the diverse Hispanic cultures. It is understood that there are the many Hispanic cultures have unique beliefs and values derived from respective locations. The study group was predominately from Mexico, aligned with the U.S. ethnic group. Thus, a limitation of the study is the small sample having limited transferability of findings. This study is a starting point for a larger program of research in this area. As such, it has highlighted an entrée into the lives of Hispanic mothers facing challenges of parenting their child with $\mathrm{CF}$, as they intersect with their family, community, and health care system.

\section{Final Considerations}

This research project embarked in an unanticipated direction, traveling through a journey to parts unknown prior to the project. Not surprisingly, mothers revealed more similarities than differences of their experiences and their practice of parenting, specifically mothering their child with CF. I was inspired by novelist, Chimamanda Ngozi Adichie (2009), whose presentation about the power of the single story brought to light an important caveat. According to Adichie, when a single story is told repetitively over time, representing a group of people, the actors in the story become that story. In other words, the creations of stereotypes emerge. She continued to discuss how the single 
story might strip dignity and humanity from the actors by representing a single facet of their life. In reality, there are many facets, many stories, yet often single stories are told that represent a negative standpoint, underscoring differences rather than similarities. The single story is subject to the power of the teller. Power exists through whom, how, and when the story is told, yet she continued to say that the single story is incomplete by making one story the story.

Likewise, for the 10 mothers in this study, this is their single story. These mothers have many stories and realities to their lives. Generalizability of this project is limited, as are stories of qualitative research, yet their stories open a window into their personal space and worlds. Some of the mutable facets and maternal reflections within the mothers' narratives can be appreciated. The mothers in the study acknowledged their transformative realities of thinking and acceptance of what was once experienced as a crisis or trauma that evolved overtime to become a normal part of their lives. For example, one mother told of her initial sense of feeling overwhelmed about learning to care for her infant with $\mathrm{CF}$; then with time transitioned to describing the care of her healthy son and daughter with $\mathrm{CF}$ as not so different. Caution exists, then, for narratives of single stories over time, that may evolve as a historic placeholder as Hispanic women find their voice to exert power to support gender, class, and cultural variations to break the pattern of the single story representations of Hispanic women. We as people hold an umbrella of humanity and are not so different. According to Chase (2005), "Any narrative is significant because it embodies - and gives us insight into- what is possible, what is intelligible within a specific context" (p. 667). 


\section{References}

Addis, M. E., \& Mahalik, J. R. (2003). Men, masculinity, and the contexts of help seeking. American Psychologist, 58(1), 5-15. doi:10.1037/0003-66X.58.1.5

Adichie, C. N. (2009). The danger of a single story. TED Talk TEDGlobal Oxford: UK Retrieved from https://www.ted.com/talks/chimamanda_adichie_the_danger_ of_a_single_story

Admi, H. (1996). Growing up with a chronic condition: A model of an ordinary lifestyle. Qualitative Health Research, 6(2), 163-183.

Allen, D. G. (1987). Critical social theory as a model for analyzing ethical issues in family and community health. Family and Community Health, 10(1), 63-72.

American College of Obstetricians and Gynecologists. (2011). Update on carrier screening for $C F$. Retrieved from http:/www.acog.org/-/media/CommitteeOpinions $/$ Committee-on-Genetics/co486.pdf?dmc $=1 \&$ ts $=20150314 T 0432349720$

Arnett, J. J. (2003, Summer). Conceptions of the transition to adulthood among emerging adults in American ethnic groups. New Directions for Child and Adolescent, 100, $63-75$.

Bailey, A. (1994). Mothering, diversity, and peace politics. Hypatia, 9(2), 189-198.

Bearison, D. J., Minian, N., \& Granowetter, L. (2002). Medical management of asthma and folk medicine in a Hispanic community. Journal of Pediatric Psychology, 27, $385-392$.

Belenky, M. F., Clinchy, B. M., Goldberger, N. R., \& Tarule, J. M. (1997). Women's ways of knowing: The development of self, voice, and mind. New York, NY: Basic Books. 
Berg, J., Anderson, N. L. R., Tichacek, M. J., Tomizh, A. C., \& Rachelefsky, G. (2007). "One gets so afraid": Latino families and asthma management—An exploratory study. Journal of Pediatric Health, 21(6), 361-371. doi:10.1016/j.pedhc.2006.08 .04

Berge, J. M., \& Patterson, J. M. (2004). Cystic fibrosis and the family: A review and critique of literature. Families, Systems, \& Health, 22(1), 74-100. doi:10.1037 $/ 1091-7527.22 .1 .74$

Berry, J. G., Bloom, S., Foley, S., \& Palfrey, J. S. (2010). Health inequity of children and youth with chronic health conditions. Pediatrics, 126(S3), S111-S119. doi:10 $.1542 /$ peds. $2010-1466 \mathrm{D}$

Besier, B., Born, T., Henrich, G., Hinz, A., Quittner, A. L., Goldbeck, L., \& the TIDES study group. (2011). Anxiety, depression, and life satisfaction in parents caring for children with cystic fibrosis. Pediatric Pulmonology, 46(7), 672-682. doi:10 $.1002 /$ ppul.21423

Bloomberg, L. D., \& Volpe, M. (2012). Completing you qualitative dissertation: A road map from beginning to end. Thousand Oaks, CA: Sage.

Bluebond-Langner, M. (1996). In the shadow of illness: Parents and siblings of the chronically ill child. Princeton NJ: Princeton University Press.

Bonilla, Z. E., \& Edwards, P. D. (2011). Difficulties encountered by health care providers serving Latino families and their children with craniofacial conditions. Hispanic Health Care International, 9, 61-72. doi:10.1891/1540-4153.9.2.61

Boss, P., \& Carnes, D. (2012). The myth of closure. Family Process, 51(4), 456-469. doi:10.1111/famp.12005 
Boss, P., \& Couden, B. A. (2002). Ambiguous loss from chronic physical illness: Clinical interventions with individual, couples, and families. Journal of Clinical Psychology, 58(11), 1351-1360. doi:10.1002/jclp.10083

Brookins, G. K. (1993). Culture, ethnicity, and bicultural competence: Implications for children with chronic illness and disability. Pediatrics, 91(5), S1056-1062.

Cabizuca, M., Mendlowicz, L., Marques-Portella, C., Ragoni, C., Coutinho, E. S. F., de Souza, W. de Jesus Mari, J., \& Figuiera, I. (2011). The invisible patients:

Posttraumatic stress disorder inpatients of individuals with cystic fibrosis. Revista de Psiquiatria Clinica, 37(1), 6-11. Retrieved from http://www.honet.usp .br/ipq/revista/vol37/n1/pdf/12.pdf

Campbell, J. C., \& Bunting, S. (1991). Voices and paradigms: Perspectives on critical and feminist theory in nursing. Advances in Nursing Science, 13(3), 1-15.

Carpenter, D. R., \& Narsavage, G. L. (2004). One breath at a time: Living with cystic fibrosis. Journal of Pediatric Nursing, 19(1), 25-32. doi:10.1016.j.pedn .2003 .09 .004

Carteret, M. (2011). Eliciting quality patient responses in cross cultural care. Retrieved from http:/www.dimensionsofculture.com/2011/03/eliciting-quality-patientresponses-in-cross-cultural-care/

Center for Disease Control and Prevention (n. d.). Asthma's impact on the nation: Data from the CDC national asthma control program. Retrieved from http://www.cde .gov/asthma/impacts_nation/asthmafactsheet.pdf

Center for Disease Control and Prevention (2014). Diabetes and youth. Retrieved from http:/www.cdc.gov/diabetes/risk/age/youth.html 
Chang, N.-Y., \& Liou, T.-Y. (2009). A study of parenting culture and practices: Listening to the voices of Latino Parents. Journal of Humanities and Social Sciences, 12, 136. Retrieved from http:/ir.hust.edu.tw/bitstream/310993100/1599/1/12-01.pdf

Charmaraman, L., \& Grossman, J. M. (2010). Importance of race and ethnicity: An exploration of Asian, Black, Latino, and multiracial adolescent identity. Cultural Diversity, 16, 144-151. doi:10.1037/a0018668

Chase, S. E. (2005). Narrative inquiry: Multiple lenses, approaches, voices. In N. K. Denzin \& Y. S. Lincoln (Eds.), The sage handbook of qualitative research (pp. 651-679). Thousand Oaks, CA: Sage.

Christian, B. J., \& D'Auria, J. P. (1997). The child's eye: Memories of growing up with cystic fibrosis. Journal of Pediatric Nursing, 12(1), 3-11.

Clandinin, D. J., \& Connelly, F. M. (2000). Narrative inquiry: Experience and story in qualitative research. San Francisco, CA: Jossey-Bass.

Clark, L., \& Redman, R. W. (2007). Mexican immigrant mothers' expectations for children's health services. Western Journal of Nursing Research, 29, 670-690. doi:10.1177/0193945906297375

Coffey, A., \& Atkinson, P. (1996). Making sense of qualitative data. Thousand Oaks, CA: Sage.

Coffey, J. S. (2006). Parenting a child with chronic illness: A meta-synthesis. Pediatric Nursing, 32, 51-59.

Collins, P. H. (2007). Shifting the center: race, class, and feminist theorizing. In A. O'Reilly (Ed.), Maternal theory essential readings (pp. 311-330). Bradford, Canada: Demeter Press. 
Corbyn, Z. (2012). Promising new era dawns for cystic fibrosis treatment. The Lancet, $379,1475-1476$.

Cortez, M. (2008). Trying to fit in a different world: Acculturation of Latino families with young children in the United States. International Journal of Early Childhood, 40(1), 97-100.

Costa, C. L., Geraldo, M. R. F., Arroteia, C. C., \& Kellelmeier, C. (2010). In vitro activity of neem oil (Azadirachta indica [A. Huss (Meliaceae)] on Aspergillus flavus growth, sporulation, viability of spores, morphology, and aflatoxins $\mathrm{B}_{1}$ and

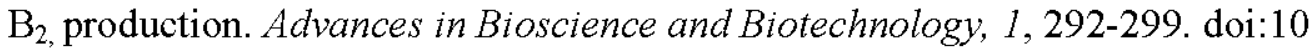
$.4236 / \mathrm{abb} .2010 .14038$

Cousino, M. K. (2013). Parenting stress among caregivers of children with chronic illness: A systematic review. Journal of Pediatric Psychology, 38(8), 809-828. doi:10.1093/jpepsy/jsto49

Creswell, J. W. (2012). Narrative research designs. In J. W. Creswell (Ed.) Educational research: Planning, conducting, and evaluating quantitative and qualitative research ( $4^{\text {th }}$ ed., Custom ed.; pp. 501-533). Boston, MA: Pearson Education.

Cronin, A. F. (2004). Mothering a child with hidden impairments. The American Journal of Occupational Therapy, 58(1), 83-92.

Cystic Fibrosis Foundation. (2012). Carrier testing for $C F$. Retrieved from http:/www .cff.org/AboutCF/Testing/Genetics/GeneticCarrierTest/

Cystic Fibrosis Foundation. (2013). Cystic Fibrosis Foundation patient registry 2012 annual data report. Bethesda MD. Retrieved from http://www.cff.org /UploadedFiles/research/ClinicalResearch/PatientRegistryReport/2012-CFF- 
Patient-Registry.pdf

Cystic Fibrosis Foundation. (2014). About $C F$. Retrieved from http://www.cff.org /AboutCF/Faqs/

Davis, P. B. (2006). History of cystic fibrosis since 1938. American Journal of Respiratory and Critical Care Medicine, 173, 475-482. doi:10.1164/rccm $.200505-840 \mathrm{OE}$

Deatrick, J. A., Knafl, K. A., \& Murphy-Moore, C. (1999). Clarifying the concept of normalization. Journal of Nursing Scholarship, 31(3), 209-214.

Denzin, N. K., \& Lincoln, E. S. (Eds.). (2005). The Sage handbook of qualitative research. Thousand Oaks, CA: Sage.

Derichs, N. (2013). Targeting a genetic defect: Cystic fibrosis transmembrane conductance regulator modulators in cystic fibrosis. European Respiratory Review, 22(127), 58-65. doi:10.1183/09059180.00008412

Devault, M. L. (1996). Talking back to sociology: Distinctive contributions of feminist methodology. Annual Review of Sociology, 22, 29-50.

Doherty, W. J., \& McCubbin, H. I. (1985). Families and health care: An emerging arena of theory, research, and clinical intervention. Family Relations, 34, 5-11.

Domenech Rodriquez, M. M., Donovick, M. R., \& Crowley, S. L. (2009). Parenting styles in a cultural context: Observations of "protective parenting" in first generation Latinos. Family Process, 48, 195-210. doi:10.1111/j.15455300.2009.01277.x

Dreby, J. (2006). Honor and virtue: Mexican parenting: Transnational context. Gender and Society, 20(1), 32-59. doi:10.1177/0891243205282660 
Elder, J. P., Broyles, S. L., Brennan, J. J., Zuniga de Nuncio, M. L., \& Nader, P. R. (2005). Acculturation, parent-child acculturation differential, and chronic disease risk factors in a Mexican-American population. Journal of Immigrant Health, 7(1), 1-9. doi:10.1007/s10903-005-1385-x

Elster, A., Jarosik, J., VanGeest, J., \& Fleming, M. (2003). Racial and ethnic disparities in health care for adolescents: A systematic review of literature. Archives of Pediatric and Adolescent Medicine, 157, 867-874.

Ennis, S. R., Rios-Vargas-M., \& Albert, N. G. (2011). The Hispanic population 2010: 2010 census briefs. United States Census Bureau. Retrieved from www.census .gov/prod/cen2010/briefs/c2010br-04.pdf

Executive order $\$ 13166$ (August 16, 2000), Retrieved from http:/www.hhs.gov/ocr /civilrights/resources/laws/revisedlep.htmlof the 1964 U.S. Civil Rights Act, 42 U.S.C. § 2000d. Office of Civil Rights, U.S. Explanation: The Executive Order requires Federal agencies to examine the services they provide, identify any need for services to those with limited English proficiency (LEP), and develop and implement a system to provide those services so LEP persons can have meaningful access to them. Department of Health and Human Services, Guidance to Federal Financial Assistance Recipients Regarding Title VI Prohibition Against National Origin Discrimination Affecting Limited English Proficient Persons, 68

Farrell, P. M., Rosenstein, B. J., White, T. B., Accurso, F. J., Castellani, C., Cutting, G. R.,...Campbell, P. W. (2008). Guidelines for diagnosis of cystic fibrosis in newborns through older adults: Cystic fibrosis foundation consensus report. 
Journal of Pediatrics, 153, S4-S14. doi:10.1016/j.peds.2008.05.005

Fiel, S. B., FitzSimons, S. \& Schidlow, D. (1994). Evolving demographics of cystic fibrosis. Seminars in Respiratory and Critical Care Medicine, 15(5), 349-355.

Flaskerud, J. H., Lesser, J., Dixon, E., Anderson, N., Conde, F., Kim, S.,...Verzemnieks, I. (2002). Health disparities among vulnerable populations: Evolution of knowledge over five decades in Nursing Research publications. Nursing Research, 51, 74-85.

Flores, G., \& The Committee on Pediatric Research. (2010). Racial and ethnic disparities in the health and health care of children. Pediatrics, 125, 979-1020. doi:10.1542 /peds.2010-0188

Forcey, L. R. (1994). Feminist perspectives on mothering and peace. In E. N. Glenn, G. Chang, \& L. R. Forcey (Eds.), Mothering: Ideology, experience, and agency (pp. 355-375). New York, NY: Routlege.

Foronda, C., \& Waite, R. (2008). Mexican American mothers' health practices for febrile children. Hispanic Health Care International, 6, 122-131. doi:10.1891/15404153.6.3.122

Foster, C. L., Byron, M. B., \& Eiser, C. (1998). Correlates of well-being of mothers with children and adolescents with cystic fibrosis. Child: Care, Health and Development, 24(1), 41-56.

Frank, A. W. ((1995). The wounded storyteller: Body, illness and ethics. Chicago, IL: The University of Chicago Press.

Freire, P. (1993). Pedagogy of the oppressed. New York, NY: Continuum.

Friedman, M. (1990). Transcultural family nursing: Application to Latino and Black 
families. Journal of Pediatric Nursing, 5(3) 214-222.

Friedman, M., Bowden, V. R., \& Jones, E. G. (2003). The Latino family. Family Nursing: Research, Theory, \& Practice (5 ${ }^{\text {th }}$ Ed.), (pp. 513-535). Upper Saddle River, NJ: Prentice Hall.

Fuligni, A. J., Tseng, V., \& Lam, M. (1999). Attitudes toward family obligations among American adolescents with Asian, Latin American, and European backgrounds. Child Development, 70, 1030-1044.

Gage, J. D., Everett, K. D., \& Bullock, L. (2006). Integrative review of parenting in nursing research. Journal of Nursing Scholarship, 38(1), 56-62.

Gallagher, M. R., Gill, S., \& Reifsnider, E. (2008). Child health promotion and protection among Mexican mothers. Western Journal of Nursing Research, 30, 588-605. doi: $10.1177 / 0193945907309752$

Gayer, D., \& Ganong, L. (2006). Family structure and mothers' caregiving of children with cystic fibrosis. Journal of Family Nursing, 12, 390-412. doi:10.1177 $/ 1074840706294510$

George, M., Rand-Giovannetti, D., Eakin, M. N., Borrelli, B., Zettler, M., \& Riekert, K. A. (2010). Perceptions of barriers and facilitators: Self-management decisions by older adolescents and adults with CF. Journal of Cystic Fibrosis, 9, 425-432. doi:10.1016/j.jcf.2010.08.016

Gibson, C. (1988). Perspective in parental coping with a chronically ill child: The case of cystic fibrosis. Issues in Comprehensive Nursing, 11, 33-41.

Gibson, C. H. (1995). The process of empowerment in mothers of chronically ill children. Journal of Advanced Nursing, 21, 1201-1210. 
Gibson, C. H. (1999). Facilitating critical reflection in mothers of chronically ill children. Journal of Clinical Nursing, 8, 305-312.

Gill-Hopple, K., \& Brage-Hudson, D. (2012). Compadrazgo: A literature review. Journal of Transcultural Nursing, 23, 117-123. doi:10.1177/1043659611433870

Gilligan, C. (1993). In a different voice: Psychological theory and women's development. Cambridge, MA: Harvard University Press.

Gjengedal, E., Rustoen, T., Wahl, A. K., \& Hanestad, B. R. (2003). Growing up and living with cystic fibrosis: Everyday life and encounters with health care and social services-A qualitative study. Advances in Nursing Science, 26(2), 149159.

Glasscoe, C., \& Smith, J. A. (2011). Unravelling complexities involved in parenting a child with cystic fibrosis: An interpretative phenomenological analysis. Clinical Child Psychology and Psychiatry, 16(2), 279-298. doi:10.1177 $/ 1359104510383207$

Glenn, E. N. (1994). Social constructions of mothering. In E. N. Glenn, G. Chang, \& L. R. Forcey (Eds.), Mothering: Ideology, experience, and agency (pp. 1-29). New York, NY: Routledge.

Gonzalez-Espada, W. J, Ibarra, M., Ochoa, E. R., \& Vargas, P. A. (2006). Perceptions of differential treatment from the viewpoints of attending physicians, residents, and Hispanic parents in Arkansas, Hispanic Health Care International, 4(3), 159-169.

Götz, I., \& Götz, M. (2000). Cystic fibrosis psychological issues. Pediatric Respiratory Reviews, 1, 121-127. doi:10.1053/prrv.2000.0033 
Grau, J. M., Azmitia, M., \& Quattlebaum, J. (2009). Parenting relational and developmental processes. In F. A. Villarruel, G. Carlo, J. M. Grau, M. Azmitia, N. J. Cabrera, \& T. J. Chahin (Eds.), Handbook of U.S. Latino psychology (pp. 153-190). Thousand Oaks, CA: Sage.

Groce, N. E., \& Zola, I. K. (1993). Multiculturalism, chronic illness, and disability. Pediatrics, 91, 1048-1055.

Gutierrez, E. R. (2007). We will no longer be silent or invisible. In A. O'Reilly (Ed.), Maternal theory: Essential readings (pp. 463-704). Toronto, Ontario: Demeter Press.

Halgunseth, L. C., Ispa, J. M., \& Rudy, D. (2006). Parental control in Latino families: An integrated literature review. Child Development, 77(5), 1282-1297.

Hayes, C. C., \& Savage, E. (2008). Fathers' perspectives on the emotional impact of managing the care of their children with cystic fibrosis. Journal of Pediatric Nursing, 23(4), 250-256. doi:10.1016/j.pedn.2007.09.002

Healthypeople. (2010). Healthy people 2020: Disparities. Retrieved from http:/www .healthypeople.gov/2020/about/Disparities About.aspx

Henneman, L., Kooij, L., Bouman, K., \& ten Kate, L. P. (2002). Personal experiences of cystic fibrosis carrier couples prospectively identified in cystic fibrosis families. Journal of Medical Genetics, 110, 324-331. doi:10.1002/ajmg.10464

Hinojosa, M. S., Knapp, C. A., Madden, V. L., Huang, I. C., Sloyer, P., \& Shenkman, E. A. (2012). Caring for children with life-threatening illnesses: Impact on white, African American, and Latino families. Journal of Pediatric Nursing, 27, 500507. doi: 10.1016/jpedn. 2011.06 .013 
Hodgkinson, R., \& Lester, H. (2002). Stress and coping strategies of mother's living with a child with cystic fibrosis: Implications for nursing professionals. Journal of Advanced Nursing, 39, 377-383.

Holloway, I., \& Wheeler, S. (2010). Qualitative research in nursing and healthcare. Oxford, UK: Wiley-Blackwell.

Hsieh, H. F., \& Shannon, S. E. (2005). Three approaches to qualitative content analysis. Qualitative Health Research, 15(9), 1277-1288. doi:10.1177/1049732305276687

Hughes, M. T., Valle-Riestra, D. M., \& Arguelles, M. E. (2008). The voices of Latino families raising children with special needs. Journal of Latinos and Education, 7(3), 241-257. doi:10.1080/15348430802100337

Hummer, R. A., \& Hamilton, E. R. (2010). Race and ethnicity in fragile families. Future of Children, 20(2), 113-131. Retrieved from http://www.futureofchildren.org /futureofchildren/publications/docs/20_02_06.pdf

Hunt, L. M. (2000). Strategic suffering: Illness narratives as social empowerment among Mexican cancer patients. In C. Mattingly \& L. C. Garro (Eds.), Narrative and the cultural construction of illness and healing (pp. 88-107). Berkeley, CA: University of California Press.

Hunter, V. (2003). The daily grind and how to stay sane as a mother of two children with cystic fibrosis. Journal of the Royal Society of Medicine, S96(43), 51-56.

Jain, M., \& Goss, C. H. (2014). Update in cystic fibrosis 2013. American Journal of Respiratory and Critical Care Medicine, 189(10), 1181-1186). doi:10.1164/rccm $.201402-0203 \mathrm{UP}$ 
Jamieson, N., Fitzgerald, D., Singh-Grewal, D., Hanson, Craig, C. S., \& Tong, A. (2104) Children's experiences of cystic fibrosis: A systematic review of qualitative studies. Pediatrics, 133(6) e1683-e1697. doi: 10.1542/peds.2014-0009

Jessup, M., \& Parkinson, C. (2010). "All at sea:" The experience of living with cystic fibrosis. Qualitative Health Research, 20, 352-364. doi:10.177 $/ 1049732309354277$

Juang, L. P., \& Umana-Taylor, A. J. (2012). Family conflict among Chinese and Mexican-origin adolescents. New Directions for Child and Adolescent Development, 135, 1-12. doi:10.1002/cd.20001

Kazak, A. E., Kassam-Adams, N., Schneider, S., Zelikovsky, N., Alderfer, M. A., \& Rourke, M. (2006). An integrative model of pediatric medical traumatic stress. Journal of Pediatric Psychology, 31(4), 343-355. doi: 10.1093/jpepsy/jsjo54

Kepreotes, E., Keatinge, D., \& Stone, T. (2010). The experience of parenting children with chronic health conditions: A new reality. Journal of Healthcare and Chronic Illness, 2, 51-62. doi:10.1111/j/1752-9824.2010.01047

Knafl, K. A., Darney, B. G., Gallo, A. M., \& Angst, D. B. (2010). Parental perceptions of the outcome and meaning of normalization. Research in Nursing and Health, 33, 87-98. doi:10.1002/nur.20367

Knafl, K. A., \& Deatrick, J. A. (1986). How families manage chronic conditions: an analysis of the concept of normalization. Research in Nursing and Health, 9, 215222. 
Knafl, K. A., Deatrick, J. A., \& Havill, N. L. (2012). Further development of the family management style framework. Journal of Family Nursing, 18(1), 11-34. doi:10 $.1177 / 1074840711427294$

Koss-Chioino, J. D., \& Vargas, L. A. (1999). Working with Latino youth: Culture, development and context. San Francisco, CA: Josey-Bass.

Kotloff, R. M., \& Zuckerman, J. B. (1996). Lung transplantation for cystic fibrosis: Special considerations. Chest, 109(3), 787-798.

Labov, W. (1972). Language in the inner city: Studies in Black English vernacular. Philadelphia, PA: University of Pennsylvania Press.

Labov, W. (1997). Some further steps in narrative analysis. Retrieved from http://www .smkb.ac.il/Eims/data2/2002/10/91153011.pdf

Labov, W. (2002). Ordinary events. Retrieved from http://www.ling.upenn.edu/ wlabov /Papers/OE.pdf

Lam, C. B., McHale, S. M., \& Updegraff, K. A. (2012). Gender dynamics in Mexican American families: Connecting mothers, fathers, and youths' experiences. Sex Roles, 67(1-2), 17-28. doi:10.1007/s11199-012-0137-3

Langellier, K. M. (1989). Personal narratives: Perspectives on theory and research. Text and Performance Quarterly, 9, 243-276.

Lanser, S. S. (1986). Toward a feminist narratology. 674-693. Retrieved from http:/www .english.uga.edu/ eberle/homepage\%20materials/lanser_femnarratology.pdf

Lieblich, A., Tuval-Mashiach, R., \& Zilber, T. (1998). Narrative research. Thousand Oaks, CA: Sage. 
Lindström, C., Åman, J., \& Norberg, A. (2010). Increased prevalence of burnout symptoms in parents of chronically ill children. Acta Paediatrica, 99, 427-432. doi:10.1111/j.1651.2227.2009.01586.x

Littlewood, J. (2011). Cystic fibrosis medicine: The history. Retrieved from http://www .cfmedicine.com/history/topics/genetics.htm

Lorenzo-Blanco, E. I., Unger, J. B., Baezconde-Garbanati, L., Ritt-Olson, A., \& Soto, D. (2012). Acculturation, enculturation, and symptoms of depression in Hispanic Youth: The roles of gender, Hispanic cultural values, and family functioning. Journal of Youth and Adolescence, 41, 1350-1365. doi:10.1007/s10964-0129774-7

Marshall, C., \& Rossman, G. B. (2011). Designing qualitative research. Thousand Oaks, CA: Sage.

Mayo, R. M., Sherrill, W. W., Sundareswaran, P., \& Crew, L. (2007). Attitudes and perceptions of Hispanic patients and health care providers in the treatment of Hispanic patients: A review of literature. Hispanic Health Care International, $5(2), 64-71$.

McLean, A. (1995). Empowerment and the psychiatric consumer/ex-patient movement in the United States: Contradictions, crisis and change. Social Science \& Medicine, $40(8)$ 1053-1071.

McClellan, C. B., \& Cohen, L.L. (2007). Family functioning in children with chronic illness compared with health controls: A critical review. Journal of Pediatrics, 50, 221-223. doi:10.1016/j.peds.2006.11.063 
McCubbin, M., Bowers, J. E., \& Holaday, B. (1984). Nursing assessment of parental coping with cystic fibrosis. Western Journal of Nursing Research, 6(4), 407-422. doi: $10.1177 / 019394598400600404$

McCubbin, H. I., Thompson, E. A., Thompson, A. I., McCubbin, M. A., \& Kaston, A. J. (1993). Culture, ethnicity, and the family: Critical factors in childhood chronic illness and disabilities. Pediatrics, 91, 1063-1070.

Meleis, A. I. (2010). Theoretical development of transitions. In A. F. Meleis (Ed.), Transition Theory: Middle-range and situation-specific theories in nursing research and practice (pp. 13-24). Retrieved from https://taskurun.files .wordpress.com $/ 2011 / 10 /$ transitions_theory_middle_range_and_situation_specifi c_theories_in_nursing_research_and_practice.pdf

Meleis, A. I., \& Im, E. (1999). Transcending marginalization in knowledge development. Nursing Inquiry, 6, 94-102.

Meltzer, L. J., \& Mindell, J. A. (2006). Impact of a child's chronic illness on maternal sleep and daytime functioning. Archives of Internal Medicine, 166, 1749-1755.

Miles, M. B., \& Huberman, A. M. (1994). Qualitative analysis: An expanded sourcebook. Thousand Oaks, CA: Sage.

Milla, C. E. (2007). Nutrition and lung disease. Clinics in Chest Medicine, 28, 319-330. doi:10.1016/jecm.2007.02.006

Mohammed, S. A. (2006). (Re)examining health disparities: Critical social theory in pediatric nursing. Journal for Specialists in Pediatric Nursing, 11, 68-71.

Moola, F. J. (2012). "This is the best fatal illness that you can have": Contrasting and comparing the experiences of parenting youth with cystic fibrosis and congenital 
heart disease. Qualitative Health Research, 22, 212-225. doi:10.177 $/ 1049732311421486$

Narrative. (n. d.). Merriam-Webster.com. Retrieved from http://www.merriamwebster.com/dictionary/narrative

Nelson, A. M. (2002). A metasynthesis: Mothering other-than-normal children. Qualitative Health Research, 12(4), 515-530.

Newacheck, P. W., Stoddard, J. J., \& McManus, M. (1993). Ethnocultural variations in the prevalence and impact of childhood chronic conditions. Pediatrics, 91, S1031-S1039.

Ngui, E. M., \& Flores, G. (2006). Satisfaction with care and ease of using health care services among parents of children with special health care needs: The roles of race/ethnicity, insurance, language, and adequacy of family centered care, Pediatrics, 117, 1184-1196. doi:10.1542/peds.2005-1088

Nicholl, H. M., \& Begley, C. M. (2012). Explicating caregiving by mothers of children with complex needs in Ireland: A phenomenological study. Journal of Pediatric Nursing, 27, 642-651. doi:10.1016/jpedn.2011.07.003

Niska, K. J. (2001). Mexican American family survival, continuity, and growth: The parental perspective. Nursing Science Quarterly, 14(4), 322-329. doi:10.1177 /08943180122108544

O'Reilly, A. (2010). $21^{\text {st }}$ century motherhood: Experience, identity, policy, agency. New York, NY: Columbia University Press.

O’Sullivan, A. K., Sullivan, J., Higuchi, K., \& Montgomery, A. B. (2011). Health care utilization and costs for cystic fibrosis patients with pulmonary infections. 
Managed Care, 37-44. Retrieved from http://www.managedcaremag.com /archives/1102/1102.peer_CFcosts.html

Overcash, J. A. (2004). Narrative research: A viable methodology for clinical nursing. Nursing Forum, 39(1), 15-22.

Owen, D. C., Gonzalez, E. W., \& Esperat, C. R. (2013). Mexican Americans. In J. N. Giger (Ed.), Transcultural nursing: Assessment \& intervention (pp. 207-239). St. Louis, MO: Elsevier Mosby.

Padilla, A. M., \& Perez, W. (2003). Acculturation, social identity, and social cognition: A new perspective. Hispanic Journal of Behavioral Sciences 25, 35-55. doi:10.1177 $/ 0739986303251694$

Padilla, Y., \& Villalobos, G. (2006). Cultural responses to health among Mexican American women and their families. Family Community Health, 30, S24-S33.

Paley, J., \& Eva, G. (2005). Narrative vigilance: The analysis of stories in health care. Nursing Philosophy, 6(2), 83-97.

Parra-Cordona, J. R., Cordova, D., Holtrop, K., Villarruel, F. A., \& Wieling, E. (2008). Shared ancestry, evolving stories: Similar and contrasting life experiences describes by foreign born and U.S. born Latino parents. Family Process, 47, $157-$ 172.

Patterson, B. (2001). Myth of empowerment in chronic illness. Journal of Advanced Nursing, 34(5), 574-581.

Patterson, J. M., McCubbin, H. I., \& Warrick, W. J. (1990). The impact of family functioning of health changes in children with cystic fibrosis. Social Science Medicine, 31(2), 159-164. 
Perry, D. F., \& Ireys, H. T. (2001). Maternal perceptions of pediatric providers for children with chronic illnesses. Maternal and Child Health Journal, 5, 15-20.

Pew Hispanic Center. (2009). Between two worlds: How young Latino's come of age in America. Washington, DC: Pew Research Center. Retrieved from http://www .pewhispanic.org/files/reports/117.pdf

Pizzignacco, T. M., Mello, D. F., \& Lima, R. A. G. (2010). Stigma and cystic fibrosis. Revista Latino-Americana de Enfermagem, 18(1), 139-142. Retrieved from http://www.scielo.br/scielo.php?script=sci_arttext\&pid=S0104$11692010000100021 \& \operatorname{lng}=\mathrm{en} \& \mathrm{nrm}=\mathrm{iso} \& \operatorname{tng}=\mathrm{en}$

Pizzignacco, T. M., Mello, D. F., \& Lima, R. G. (2011). The experience of disease in cystic fibrosis: The paths to comprehensive care. Revista da Escola de Enfermagem da USP 45(3), 636-42. Retrieved from http:/www.scielo.br/pdf /reeusp/v45n3/en_v45n3a13.pdf

Polit, D. F., \& Beck, C. T. (2012). Nursing research: Generating and assessing evidence for nursing practice. Philadelphia, PA: Wolters Kluwer/Lippincott Williams \& Wilkins.

Polkinghorne, D. E. (1988). Narrative knowing and the human sciences. Albany, NY: State University of New York Press.

Quinones-Mayo, Y., \& Dempsey, P. (2005). Finding the bicultural balance: Immigrant Latino mother raising "American" adolescents. Child Welfare, LXXXIV, 649-667. Quintana, S. M., \& Scull, N. C. (2009). Latino ethnic identity. In F.A. Villarruel, G. Carlo, J. M. Grau, M. Azmitia, N. J. Cabrera, \& T. J. Chahin (Eds.), Handbook of U.S. Latino psychology (pp. 81-98). Thousand Oaks, CA: Sage. 
Quinton, P. M. (1999). Physiological basis for cystic fibrosis: A historical perspective. Physiological Reviews, 29(1), S3-S22.

Quittner, A. L., Schechter, M. S., Rasouliyan, L., Haselkorn, T., Pasta, D. J., \& Wagener, J. S. (2010). Impact of socioeconomic status, race, and ethnicity on quality of life in patients with cystic fibrosis in the United States. Chest, 137, 642-650. doi:10 $.1378 /$ chest.09-0345

Radosh, P. F. (2008). Sara Ruddick's theory of maternal thinking applied to traditional Irish mothering. Journal of Family History, 33(3), 304-315. doi:10.1177 $/ 0363199008318918$

Ray, L. D. (2002). Parenting and childhood chronicity: Making visible the invisible work. Journal of Pediatric Nursing, 17, 424-438. doi:10.1053.jpdn.2002.127172

Rehm, R. S. (1999). Religious faith in Mexican-American families dealing with chronic childhood illness. Image: Journal of Nursing Scholarship, 31(1), 33-38.

Rehm, R. S. (2000). Parental encouragement, protection, and advocacy for MexicanAmerican children with chronic conditions. Journal of Pediatric Nursing, 15, 8998. doi:10.1053/jn.20000.5448

Rehm, R. S. (2003). Cultural intersection in the care if Mexican-American children with chronic conditions. Pediatric Nursing, 29, 434-439.

Riessman, C. K. (1987). When gender is not enough: Women interviewing women. Gender \& Society, 1, 172-207. doi:10.1177/0891243287001002004

Riessman, C. (1989). Life events, meaning and narrative: The case of infidelity and divorce. Social Science and Medicine, 29(6), 743-751. 
Riessman, C. (1990), Divorce talk: Women and men make sense of personal relationships. New Brunswick, NJ: Rutgers University Press.

Riessman, C. K. (1993). Narrative analysis. Newbury Park, CA: Sage.

Riessman, C. K. (2008). Narrative methods for the human sciences. Thousand Oaks, CA: Sage.

Robledo, L., Wilson, A. H., \& Gray, P. (1999). Hispanic mother's knowledge and care of their children with respiratory illnesses: A pilot study. Journal of Pediatric Nursing, 14, 239-247.

Rohlfs, E. M., Zhou, Z., Heim, R. A., Nagan, N., Rosenblum, L. S., Flynn, K.,...Sugarman, E. A. (2011). Cystic fibrosis carrier testing in an ethnically diverse U.S. population. Clinical Chemistry, 57, 841-848. doi:10.1373/clinchem .2010 .159285

Rolland, J. S. (1994). Families, illness, \& disability. New York, NY: Basic Books.

Rommens, J. M., Iannuzzi, M. C., Kerem, B. S., Drumm, M. L., Melmer, G., Dean, M.,...Collins, F. S. (1989). Identification of the cystic fibrosis gene:

Chromosomes walking and jumping. Science, 245, 1059-1065.

Rothman, B. K. (1994). Beyond mothers and fathers: Ideology in a patriarchal society. In E. N. Glenn, G. Chang, \& L. R. Forcey (Eds.), Mothering: Ideology, experience, and agency (pp. 139-157). New York, NY: Routlege.

Rowe, S. M., Borowitz, D. S., Burns, J. L., Clancy, J. P., Donaldson, S. H., RetschBogart, G.,...Ramsey, B. W. (2012). Progress in cystic fibrosis and the CF therapeutics development network. Thorax, 67, 882-890. doi:10.1136/thoraxjnl2012-202550 
Rowland, E., \& Metcalfe, A. (2013). Communicating inherited genetic risk between parent and child: A meta-thematic synthesis. International Journal of Nursing Studies, 50, 870-880. doi: 10.1016/j.inurstu.2012.09.002

Ruddick, S. (1995). Maternal thinking: Toward a politics of peace. Boston, MA: Beacon Press.

Ruddick, S. (2007). Maternal thinking. In A. O'Reilly (Ed.), Maternal theory: Essential readings (pp. 96-113). Bradford, Canada: Demeter Press.

Rueda, R., Monzo, L., Shapiro, J., Gomez, J., \& Blacher, J. (2005). Cultural models of transition: Latina mothers of young adults with developmental disabilities. Exceptional Children, 71, 401-414.

Sanchez-Birkhead, A. C., Kennedy, H. P., Callister, L. C., \& Miyamoto, T. P. (2011). Navigating a new health culture: Experiences of immigrant Hispanic women. Journal Immigrant Minority Health, 13, 1168-1174. doi:10.1007/s10903-0109369-x

Sandelowski, M. (1991). Telling stories: Narrative approaches in qualitative research. Image: The Journal of Nursing Scholarship, 23, 161-166.

Sandelowski, M., \& Barroso, J. (2003). Writing the proposal for a qualitative research methodology project. Qualitative Health Research, 13, 781-820. doi:10.1177 /1049732303013006003

Sandoval, A. M. (2008). Toward a Latina offeminism in the Americas: Repression and resistance in Chicana and Mexicana literature. Austin, TX: University of Texas Press. 
Schechter, M. (2011). Nongenetic influences on cystic fibrosis outcomes. Current Opinion in Pulmonary Medicine, 17, 448-454.

doi:10.1097/MCP.0b013e32834ba899

Schwandt, T. A. (2007). The Sage dictionary of qualitative inquiry. Thousand Oaks, CA: Sage.

Smedley, B., Stith, A., Nelson, A., \& Committee on Understanding and Eliminating Racial and Ethnic Disparities in Health Care. (2002a). What healthcare consumers need to know about racial and ethnic disparities in healthcare. Retrieved from http://www.iom.edu/ /media/Files/Report\%20Files/2003 /Unequal-Treatment-Confronting-Racial-and-Ethnic-Disparities-in-HealthCare/PatientversionFINAL.pdf

Smedley, B., Stith, A., Nelson, A., \& Committee on Understanding and Eliminating Racial and Ethnic Disparities in Health Care. (2002b). Unequal treatment: What healthcare providers need to know about racial and ethnic disparities in healthcare. Retrieved from http:/www.iom.edu/ /media/Files/Report\%20Files /2003/Unequal-Treatment-Confronting-Racial-and-Ethnic-Disparities-in-HealthCare/Disparitieshcproviders8pgFINAL.pdf

Smith, B. A., Modi, A. C., Quittner, A. L., \& Wood, B. L. (2010). Depressive symptoms in children with cystic fibrosis and parents and its effects on adherence to airway clearance. Pediatric Pulmonology, 45, 756-763. doi:10.1002/ppul.21238

Smith-Morris, C., Morales-Campos, D., Castaneda Alvarez, E. A., \& Turner, M. (2013). An anthropology of familismo: On narratives and description of 
Mexican/Immigrants. Hispanic Journal of Behavioral Sciences, 35(1), 35-60. doi: $10.1177 / 0739986312459508$

Spector, R. E. (2004). Cultural diversity in health and illness. Upper Saddle River, NJ: Pearson Prentice Hall.

Strom, R. D., Strom, P. S., \& Beckert, T. E. (2008). Comparing Black, Hispanic, and White mothers with a national standard of parenting. Adolescence, 43, 525-545.

Studer, Q., Robinson, B. C., \& Cook, K. (2010). The HCAHPS handbook. Gulf Breeze, FL: Fire Starter.

Sullivan-Bolyai, S., Knafl, K. A., Sadler, L., \& Gilliss, C. L. (2004). Great expectations: A position description for parents as caregivers: Part II. Pediatric Nursing, 30, $52-56$.

Sullivan-Bolyai, S., Sadler, L., Knafl, K. A., \& Gilliss, C. L. (2003). Great expectations: A position description for parents as caregivers: Part I. Pediatric Nursing, 29, $457-461$.

Szyndler, J. E., Towns, S. J., van Asperen, P. P., \& McKay, K. O. (2005). Psychological and family functioning and quality of life in adolescents with cystic fibrosis. Journal of Cystic Fibrosis, 4, 135-144. doi:10.1016/j.jef.2005.02.004

Tanase, A., \& Zanni, R. (2008). The use of complementary and alternate medicine among pediatric cystic fibrosis patients. The Journal of Alternative and Complementary Medicine, 14 (10). 1271- 1273. doi: 10.1089/1cm.2008.0185

Tavares, K. O., Carvalho, M. D., \& Pelloso, S. M. (2010). What is it like to be the mother of a child with cystic fibrosis [Abstract]. Revista Gaucha de Enfermagem, 31, 723-729. Retrieved from http://www.ncbi.nlm.nih.gov/pubmed/21805883 
Taylor, P., \& Cohn, D. (2012). A milestone en route to a majority minority nation. Pew Research Social and Demographic Trends, Pew Hispanic Center. Retrieved from http://www.pewsocialtrends.org/2012/11/07/a-milestone-en-route-to-a-majorityminority-nation/

Thomson, M. D., \& Hoffman-Goetz, L. (2009). Defining and measuring acculturation: A systematic review of public health studies with Hispanic populations in the United States. Social Science \& Medicine, 69, 983-991. doi:10.1016/j.socscimed .2009 .05 .011

Thomson (Ed.). (2004). Physicians' Desk Reference for herbal medicine. ( $3^{\mathrm{rd}} \mathrm{ed}$.), pp. 604-606, 609-610). Montvale, NJ. PDR Network.

Thursfield, R. M., \& Davies, J. C. (2012). Cystic fibrosis: Therapies targeting specific gene defects. Pediatric Respiratory Reviews, 13, 215-219. doi:10.1016/j.prrv .2012 .04 .003

Tracy, J. P. (1997). Growing up with chronic illness: The experience of growing up with cystic fibrosis. Holistic Nursing Practice, 12(1), 27-35.

Updegraff, K. A., \& Umana-Taylor, A. (2015). What can we learn from the study of Mexican-Origin families in the United States? Family Process. Advance online publication. doi: $10.1111 /$ famp. 12135

Varela, R. E., Vernberg, E. M., Sanchez-Sosa, J. J., Riveros, A., Mitchell, M., \& Mashunkasjey, J. (2004). Parenting style of Mexican, Mexican American, and Caucasian non-Hispanic families: Social context and cultural influences. Journal of Family Psychology, 18, 651-657. doi:10:1037/0893-3200.18.4.651 
Vasquez, J. M. (2010). Chicana mothering in the twenty-first century: Challenging stereotypes and transmitting culture. In A. O'Reilly (Ed.), $21^{\text {st }}$ century motherhood: Experience, identity, policy, agency (pp. 23-39). New York, NY: Columbia University Press.

Vega, W. A. (1990). Hispanic families in the 1980's: A decade of research. Journal of Marriage and Family, 52, 1015-1024.

Viner, R. M., Ozer, E. M., Denny, S., Marmot, M., Resnick, M., Fatusi, A., \& Currie, C. (2012). Adolescence and social determinants of health. Lancet, 379, 1641-1652. doi:10.1016/S0140-6736(12)60149-4

Virasiri, S., Yunibhand, J., \& Chaiyawat, W. (2011). Parenting: What are the critical attributes? Journal of the Medical Association of Thailand, 94, 1109-1116.

Wallace, P. M., Pomery, E. A., Latimer, A. E., Martinez, J. L., \& Salovey, P. (2010). A review of acculturation measures and their utility in studies promoting Latino health. Hispanic Journal of Behavioral Sciences, 32, 37-54.

Watts, K. D., Seshadri, R., Sullivan, C., \& McColley, S. A. (2009). Increased prevalence of risk factors for morbidity and mortality in the U.S. Hispanic CF population. Pediatric Pulmonology, 44, 594-601. doi:10.1002/ppul.21037

Wells, K. (2011). Narrative inquiry. New York, NY: Oxford University Press

Williams, B., Corlett, J., Dowell, J. S., Coyle, J., \& Mukhopadhyay, S. (2009). "I've never not had it so I don't really know what it's like not to": Nondifference and biographical disruption among children and young people with cystic fibrosis. Qualitative Health Research, 19, 1443-1455. doi:10.1177/1049732309348363 
Wolfenden, L. L., \& Schechter, M. S. (2009). Genetic and non-genetic determinants of outcomes in cystic fibrosis. Paediatric Respiratory Reviews, 10, 32-26. doi:10 $.1016 /$ j.prrv.2008.04.002

Wong, M. G., \& Heriot, S. A. (2007). Parents of children with cystic fibrosis: How they hope, cope, and despair. Child: Care, Health, and Development, 34(3), 344-354. doi:10.1111/j.1365-2214.2007.00804.x

Xu, K. T., Borders, T. F., \& Arif, A. A. (2004). Ethnic differences in parents' perceptions of participatory decision-making style of their children's physicians. Medical Care, 42, 328-335. doi:10.1097/01.mlr.0000118707.99818.cc

Yilmaz, O., Sogut, A., Gulle, S., Can, D., Etran, P., \& Yuksel, H. (2008). Sleep quality and depression-anxiety in mothers of children of two chronic respiratory diseases: Asthma and cystic fibrosis. Journal of Cystic Fibrosis, 7, 495-500. doi:10.1016/j.jcf.2008.05.002

Zambrana, R. E., \& Dorrington, C. (1998). Economic and social vulnerability of Latino children and families by subgroup: Implications for child welfare. Child Welfare, $77(1), 5-27$.

Zoucha, R. D. (1998). The experience of Mexican Americans receiving professional nursing care: An ethnonursing study. Journal of Transcultural Nursing, 9, 34-44. doi:10.1177/104365969900900206 


\title{
Appendix A
}

Request for Waiver of Authorization for Use and Disclosure of Protected Health

Information

WAIVER OF AUTHORIZATION

PI: L. Tirabassi-Mathis RN PhD(c) MN CPNP

Page 1 of 2

Projed No: $361-14$

\section{MemorialCare Health System Institutional Review Board \\ Request for Waiver of Authorization For the Use and Disclosure of Protected Health Information in Research}

\begin{abstract}
INTRODUCTION
Patients have rights regarding the privacy of their medical information collected prior to and in the course of this research. This medical information, called "protected health information" (PHI), includes demographic information, the results of histories and physical exams. blood tests, $x$-rays and other diagnostic and medical procedures, as well as individually identifiable tissues or body fluids. Patients must authorize the use of PHI when used or disclosed for all purposes other than treatment, payment and healthcare operations. This includes the use and disclosure of PHI for research. In some cases, it is appropriate to seek a waiver of such authorization, such as for chart reviews and data registries.
\end{abstract}

\section{REQUEST FOR WAVER}

I. Linda Tirabassi-Mathis. RN, PhD(c), MN CPNP, request a waiver of authorization for the use and disclosure of protected health information, consequent to a research project entifled, "Hispanic Mothers' Stories: Narratives of Mothers Experiences Parenting a Child with Cystic Fibrosis (CF). Health Beliefs, and Health Care Experiences."

The waiver of authorization for use and disclosure of PHI is for a period of 5 years after study dosure.

The specific elements of $\mathrm{PHt}$ that will be used and disclosed include name, medical record number, telephone number, all elements of dates (except year) directly related to an individual, and biometrics (forced expiralory volume in one second [FEV,], confirmation of CF by sweat test results or DNA mutation analysis). Other information collected include ages of parents and child with CF and medical diagnosis,

The members of the research team at MemorialCare Health Services who will be using the described PHI include Linda Tirabassi-Mathis, RN, PhD(c). MN CPNP; Peggy Kalowes RN. PhD. CNS, FAHA, and (when used for interpreting) Lourdes Marquez, Child Life Assistant.

I further certily the following:

yim The use or disclosure of PHI involves no more than minimal risk to the privacy of individuals, based on the presence of the following elements:

Pheaso Inilial

1. An adequate plan to protect the identifiers from improper use and disclosure.

All study materials will be coded with a subject ID number and pseudo-names assigned, thus protecting privacy. All study materials will be stored in a locked cabinet only accessible to the researchers or a password protected computer. 
WAIVER OF AUTHORIZATION

PI: L. TlrabassHMathks RN PhD(c) MN GPNP

Page 2 of 2

Project No: $381-14$

2. An adequate plan to destroy the Identifiers at the earliest opportunity consistent with conduct of the research, unless there is a specific health or research justification for retaining the identifiers, or such retention is otherwise required by law:

All transcrlbed materials, field notas, demographic forms, and log will be disposed in a confidentlal paper dlsposal bin, in the event this is unevallable all paper forms will be ehredded, In addition, all information tored In a locked computer will be completely deleted, including any portable or back up devlces. All audio taped intervierwe will be deleted and the tepes destroyed.

and

3. The PHI will not be reused or disclosed to any other person or entlyy, except as required by law, for authorlzed oversight of the research project, or for other research for which the use ar disclosure of PHI may be subsequently permitted.

$y$ m

The research could not practicably be conducted without the waiver; and

Prosege initigl

$\frac{17}{1}$ The research could not practicably be conducted without access to and use of the PHI.

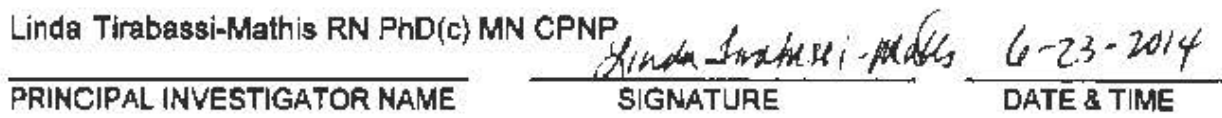

\section{FOR USE OF THE OFFXE OF RESEARCH ADMINISTAATION ONLY}

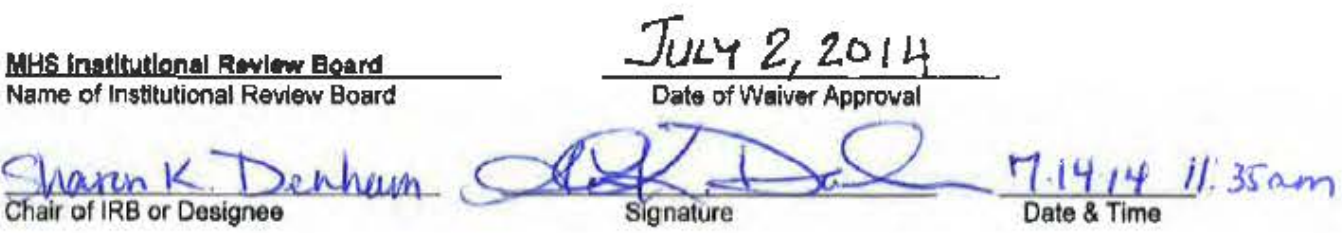

Form Rev: 3-17-2014. Supercedes all previous versions. 
Appendix B

\section{Recruitment Script}

Hello my name is Linda Tirabassi-Mathis. I am a nurse and also nursing doctoral student at the University of San Diego, I am also employed by Miller Children's \& Women's Hospital Long Beach and have a long time interest in working with children with CF and their families.

I am interested in studying what mothers from Hispanic ethnicities experience parenting your child with CF. In addition, I am also interested about your health beliefs and health care experiences related to CF. My study is an effort to represent Hispanic mother's perspectives.

The study will involve a face to face, audio taped, interview and I will ask you to "tell me your story about parenting your child with CF." The interview will take about 60 to 90 minutes. I will be happy to meet with you in a location of your choice, your home, the hospital, or another location of your convenience. Interpreters are available if you wish to have one present.

Your participation is voluntary. If you choose to participate or decline it will not affect your child's health care in any way.

Would you be interested and willing to be a part of this study? 


\begin{tabular}{|c|c|c|c|c|c|c|c|c|c|c|c|c|c|}
\hline HIS & ANIC MOTH & STORIES: & RRAT & $\begin{array}{l}\text { OF PA } \\
\text { AND } \\
\text { SCI }\end{array}$ & $\begin{array}{l}\text { TING E) } \\
\text { LTH EXI } \\
\text { NING A }\end{array}$ & $\begin{array}{l}\text { EIENCES } \\
\text { RIENCES RE } \\
\text { ENROLLEN }\end{array}$ & $\begin{array}{l}\text { CHILDRE } \\
\text { ATED TO } \\
\text { ENT LOG }\end{array}$ & $\begin{array}{l}\text { V WITH CYS } \\
\text { IF }\end{array}$ & FIBR & IS (CF), & ALTHE & LIEFS, & \\
\hline$\stackrel{w}{\frac{\pi}{\sigma}}$ & $\begin{array}{c}\text { Mother's } \\
\text { Name }\end{array}$ & $\begin{array}{l}\text { Child's } \\
\text { Name and } \\
\text { Age }\end{array}$ & 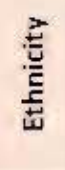 & $\begin{array}{l}\text { Age at } \\
\text { CF DX }\end{array}$ & 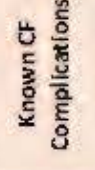 & $\begin{array}{c}\text { Additional } \\
\text { diagnosis } \\
Y / N^{*}\end{array}$ & $\begin{array}{c}\text { Eligible } \\
Y / N\end{array}$ & $\begin{array}{l}\text { Enrollment } \\
\qquad A / D^{* *}\end{array}$ & ID\# & $\begin{array}{l}\text { Appt. } \\
\text { Date/ } \\
\text { Time }\end{array}$ & $\begin{array}{l}\text { Appt. } \\
\text { place }\end{array}$ & 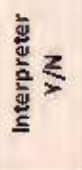 & $\frac{n}{0}$ \\
\hline & & & & & & & & & & & & & \\
\hline & & & & & & & & & & & & & \\
\hline & & & & & & & & & & & & & \\
\hline & & & & & & & & & & & & & \\
\hline & & & & & & & & & & & & & \\
\hline & & & & & & & & & & & & & \\
\hline & & & & & & & & & & & & & \\
\hline & & & & & & & & & & & & & \\
\hline & & & & & & & & & & & & & \\
\hline & & & & & & & & & & & & & \\
\hline & & & & & & & & & & & & & \\
\hline & & & & & & & & & & & & & \\
\hline & & & & & & & & & & & & & \\
\hline & & & & & & 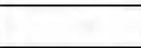 & 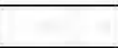 & 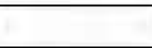 & & & & & \\
\hline & & & & & & & & & & & & & \\
\hline & & & & & & & & & & & & & \\
\hline & & & & & & & & & & & & & \\
\hline & & & & & & & & & & & & & \\
\hline
\end{tabular}


Appendix D

Appendix D

CF Newsletter Recruitment Announcement

RECRUITMENT ANNOUNCEMENT: CF Newsletter, IRB \# 361-14

CALLING ALL HISPANIC/LATINO MOTHERS: WOULD YOU LIKE TO TELL YOUR STORY OF WHAT IT IS LIKE TO BE A PARENT OF YOUR CHILD WITH CYSTIC FIBROSIS (CF)?

A nurse researcher who has a long time interest with CF is conducting a study, as a graduate student, to explore and describe Hispanic/Latino mothers' experiences about what it is like to be a parent to your child with CF. The study will also explore and describe your health beliefs, practices, and experiences related to your child with $C F$.

All participation is voluntary. Participation in this study will help the field of nursing to understand how to better serve Hispanic/Latino families.

To qualify to participate in this study you must:

1. Be of Hispanic/ Latino heritage

2. Have a biological child with $\mathrm{CF}$

3. Be older than 18 years of age

4. May speak Spanish* or English (*Interpreters are available)

5. Your child must have a diagnosis of CF for at least one year, without other diagnoses, except other problems known to be part of $\mathrm{CF}$

What You Will Be Asked To Do?

Willing participants in this study will participate in a 60 and 90 minute face to face interview with the researcher regarding your experiences of parenting your child with CF from your Hispanic/ Latino perspective. The interview will be audio recorded for research purposes. You may be contacted at a later date to verify your story for an additional 30 minutes.

If you choose to participate, all information will be kept confidential. All participants in the study will receive $\$ 20$ reimbursement check for participation at the completion of the interview(s).

If you have any questions or would like to participate in my study, please call or email me at:

562-933-0614 or email at ltirabassi@memorialcare.org

Your interest and participation are greatly appreciated.

Thank you.

Linda Tirabassi-Mathis RN PhD(c) MN CPNP

Principal Investigator MHS IRB \#361-14

Phone 562-933- 0614 or ltirabassi@memorialcare.org 


\section{HISPAINC MOTHERS' STORIES: NARRATIVES OF PARENTING A CHILD WITH CYSTIC}

FIBROSIS (CF), HEALTH BELIEFS, and HEALTH CARE EXPERIENCES

\section{How Can I Be Part of This Study?}

Be a mother of Hispanic ethnicity

- Have a child with cystic fibrosis (CF)

- Willing to tell your story about parenting your child with CF

- Take about 60-90 minutes of your time in a location of your choice to tell your story (interpreters are available)

장 child:

- Must have a diagnosis of CF for at least 1 year

- Be between the ages of 1 and 21 years of age

Linda Tirabassi-Mathis RN PhD(c) MN CPNP is a graduate student in the Hahn School of Nursing and Health Sciences at the University of San Diego. She is also an employee of Miller Children's Hospital Long Beach

\section{WHY IS THIS STUDY IMPORTANT?}

Mothers are usually the main caregivers of children. It is important to

understand your experiences and

viewpoint in taking care of your child with CF.

9. $\mathrm{CF}$ is detected in Hispanic individuals less often than in Caucasians. Since the Hispanic population is the fastest growing minority in the United States, more infants and children may be identified with $\mathrm{CF}$ in the future.

(3) Learning directly from mothers about your experiences will help to improve the care for Hispanic children with CF and their families today and in the future.

(a) Information from this study will help to educated nurses and CF health care teams. It will be used develop strategies to improve the care and health of Hispanic children with $\mathrm{CF}$ and their families.

\section{PLEASE CONTACT}

Linda Tirabassi-Mathis RN PhD(c) MN

CPNP

\section{Phone: 562-944-0614}

\section{OR email:}

Itirabassi@memorialcare.org

Thank You 
Appendix $F$

Informed Consent to Participate in a Research Study

MemorialCare Health System Institutional Review Board

\section{INFORMED CONSENT TO PARTICIPATE IN A RESEARCH STUDY}

Title: Hispanic Mothers' Narratives: Experiences of Parenting a Child with
Cystic Fibrosis (CF), Health Care Beliefs, and Health Care Experiences
Related to CF

Principal Investigator: Linda Tirabassi-Mathis RN PhD(c) Phone: 562-933-0614 MN CPNP

Sub-Investigator: Peggy Kalowes RN PhD CNS FAHA Phone: 562-933-5016

Clinical Study Team: Lourdes Marquez, Child Life Phone: 562-933-8060 Assistant - Interpreter

MHS Project No: $\quad 361-14$

Sponsor: Memorial Medical Center Foundation

You have been asked to take part in a research study. We want you to know that taking part in a research study is entirely voluntary.

\section{Purpose of the Study:}

You are being asked to participate in a research study conducted by Linda TirabassiMathis RN, PhD(c), MN, a doctoral student at the University of San Diego Hahn School of Nursing and Health Sciences, who is conducting her dissertation research at the Miller Children's \& Women's Hospital Long Beach (MCWH) cystic fibrosis (CF) outpatient clinic. She is also an employee of the hospital, which is part of the MemorialCare Health System.

The purpose of this research study is to describe and explore perceptions of Hispanic mothers who have a child with cystic fibrosis related to your parenting experiences, cultural health beliefs and health care experiences related to $\mathrm{CF}$. Learning directly from mothers is the best way to get this information. This consent form is intended to describe the research study and your responsibilities as a research participant as well as the risks and benefits of participation. 
Page 2 of 8

PI: L. Tirabassi-Mathis RN PhD(c) MN CPNP

Project No. 361-14

\section{Description of the Study}

The goal is to recruit 20 study mothers from the MCWH CF outpatient pulmonary/ CF clinic.

Taking part in this research study is entirely voluntary. You may decide not to participate, or if you cecide to participate you may withdraw from the study at any time, without consequences of any kind. Participating or not participating will not affect the care your child receives from your physician, your health care team, or MCWH. If you decide to withdraw from the study, you are asked to contact the researcher and irform her of your decision without any additional questions asked. If at any time the researcher feels that further participation in this study is not in your best interest, he or she may withdraw you from the study.

\section{Physical Requirements}

If you agree to participate in this study, you will be asked to complete a face to face interview that may take up to 90 minutes of your time. The interview will be audiotaped for research purposes. Interpreters will be available. General questions will be asked and additional questions to clarify or expand on a description provided. Example questions include:

- Tell me your story of what your life is like being a parent to your child with CF.

- What makes it easier? / What makes it more difficult?

- What part of being a parent to your child with CF is different or the same as being a parent to your other children (or extended relatives, for an only child?)

- Tell me what does your child having CF mean to you?

- Tel me what are the issues that Hispanic mothers experience as having the greatest influence on parenting your child with CF?

- Tel me what gets you through each day?

- What is it like to think about [child's name] future with CF?

- What do you understand to be the cause of [child's name] CF?

- What do you understand about how to treat CF or what cures it? 
Page 3 of 8

PI: L. Tirabassi-Mathis RN PhD(c) MN CPNP

Project No. 361-14

- Some parents tell us they try remedies from their "home country", what have you tried?

- What has your experience been with the health care for CF that [child's name] receives?

- Can you describe a situation where you felt support related to the health care of your child?

- Related to (child's name) health care, some Hispanic parents say they have felt or experienced discrimination, I am wondering what your experience has been with your child's CF care?

- What would you like me to [most] remember and write about from our talk today?

You will also be asked to complete a general information form that will take about 8 minutes to complete which includes:

- Mother's information will include: primary caregiver, if mom works outside the home, highest education level, recall of genetic counseling;

- Primary language spoken at home, self-identified ethnicity, country of origin, insurance type, and household members who smoke tobacco; your household members and their family relationship, and their ages, including others with CF;

- The child's information obtained from the medical chart includes: child's age and age when CF was diagnosed, results of the most recent lung function (FEV ${ }_{1}$ ) and confirmation of diagnostic studies of your child's CF (sweat chloride and CF DNA results).

You will need to be able to sit for a period of time during the interview. You may take breaks as needed. You may be asked to be contacted at a later time to confirm your story and information.

\section{Duration of the Study}

All information shared in the interview will be confidential without your name, your child's name or other identifying information. All of the information shared will be kept in locked storage. It is possible the study may take up to 6 months to finish. Any shared information will stay confidential during this time and for 5 years afterward the study is finished for possible further research from only the study. During the write up of the study fake names will be given to each participant to make for easier reading. The investigator may contact 
Page 4 of 8

PI: L. Tirabassi-Mathis RN PhD(c) MN CPNP

Project No. 361-14

you, the participant, after the initial interview to check that the shared information is accurate.

\section{Reimbursement}

Upon completion of the full interview, you will be provided a $\$ 20$ reimbursement check toward transportation expenses. The full interview means the initial interview and after the final "checking in" interview to make sure your story is reported accurately. You may be contacted for a "final checking in" to be sure your shared information is accurately recorded within $30-60$ days or perhaps up to 90 days after the initial interview. The reimbursement check will be sent at that time.

\section{Risks/Side Effects}

There are minimal risks to you for participating in this study. Sometimes, individuals may feel sad or distressed when they remember or talk about their experiences. If at any time during the study you find that you do not wish to participate you may refuse to continue. A referral will be offered and made available to your community mental health center that is most convenient for you or you may call directly. The phone numbers for LA and Orange County are provided below.

MENTAL HEALTH RESOURCES

\begin{tabular}{|c|c|c|c|c|}
\hline County & $\begin{array}{c}\text { Service } \\
\text { Name }\end{array}$ & Hours & Phone Number & Language \\
\hline LA County & $\begin{array}{c}\text { Access } \\
\text { Helpline }\end{array}$ & $\begin{array}{c}24 \text { hours/day } \\
7 \text { days/week }\end{array}$ & $800-854-7771$ & $\begin{array}{c}\text { English and } \\
\text { Spanish }\end{array}$ \\
\hline $\begin{array}{c}\text { Orange } \\
\text { County }\end{array}$ & $\begin{array}{c}\text { Crisis } \\
\text { Prevention } \\
\text { Hotline }\end{array}$ & $\begin{array}{c}24 \text { hours/day } \\
7 \text { days/week }\end{array}$ & $877-727-4747$ & $\begin{array}{c}\text { English and } \\
\text { Spanish }\end{array}$ \\
\hline
\end{tabular}

\section{Potential Benefits}

Although you may not benefit personally from this study, your participation will help families and children with CF from Hispanic ethnicities by giving insight to nurses and to health care providers of $\mathrm{CF}$ to better serve the future health needs of Hispanic children with $\mathrm{CF}$ and their families.

\section{Alternatives}

Your participation in this study is voluntary. You may choose to not participate. Your child's medica treatment is unrelated to this study. You have been given the opportunity to ask questions which have been answered to your satisfaction. Your study investigator can answer any questions that you might have in the future. 
Page 5 of 8

PI: L. Tirabassi-Mathis RN PhD(c) MN CPNF

Project No. 361-14

\section{Costs and Payments}

You or your third party payer (health insurance, Medicare, Medi-Cal or other) must provide payment for hospital, office and other medical costs unrelated to this study. You will receive no financial gain for your participation in this study. There will be no additional costs to you.

\section{Physical Injury Statements}

In the event of physical injury, medical treatments will be available but will not be provided free of charge, nor will financial compensation be provided. If you sustain an injury, you should contact the principal investigator immediately.

\section{Voluntary Participation/ Right to Withdrawal}

Your participation in this study is voluntary. You may decide not to participate or you may withdraw from the study at any time, without penalty or loss of benefits in your medical care to which you might otherwise be entitled. If you do decide to leave the study, you are asked to contact the primary investigator of the study, Linda Tirabassi-Mathis RN and inform ter of your decision.

\section{Confidentiality}

Any information about you obtained from this research will be kept confidential and your name will never be identified in any report or publication unless you sign a release. Information regarding your medical records, just like hospital records, may be subpoenaed by court order. You consent to the publication of study results so long as the information is anonymous and/or disguised so that identification cannot be made. Authorized representatives of the Food and Drug Administration (FDA), the Office of Human Research Protections (OHRP), and the MHS Institutional Review Board (IRB) may examine your medical records, and there will be no breach of confidentiality.

A descrption of this clinical trial will be available on http $/ / \mathrm{wWw}$.Clin calTrials.gov, as required by U.S. law. This Web site will not include information that can identify you. At most, the Web site will include a summary of the results. You can search this Web site at any time.

\section{IRB-FDA Clause}

This proposal has been reviewed and approved by the MHS Institutional Review Board (IRB) for the MemorialCare Health System; this board is composed of physicians and lay persons. If you have any questions about your rights as a research subject, or regarding a treatment related injury, or desire further information concerning the availability of compensation or medical treatment, you may contact MemorialCare Research at (562) 933-5600. 
Page 6 of 8

PI: L Tirabassi-Mathis RN PhD(c) MN CPNP

Project No. $361-14$

Linda Tirabassi-Mathis RN PhD(c) MN CPNP and/or Peggy Kalowes RN PhD CNS FAHA (her sub-investigator) have discussed this study with you. If you have any questions you can reach them at 562-933-0614.

I certify that I have read the preceding or it has been read to me, that I have reviewed its contents, and that any question I have pertaining to the preceding have been, or will be answered by my doctor and that my permission is freely given. I have been given a signed copy of this consent form along with a copy of the "Rights of Human Subjects in Medical Research," and I consent to participate in this study.

Printed Name of Subject

Subject's Signature

Date Time

If the subject is a minor, or otherwise unable to sign, complete the following:

a) Reason subject is unable to sign:

b)

Printed Name of Authorized Person

Signature of Authorized Person

Relationship and Basis of Authorization to Give Consent
Date

Time

Subject's Initials

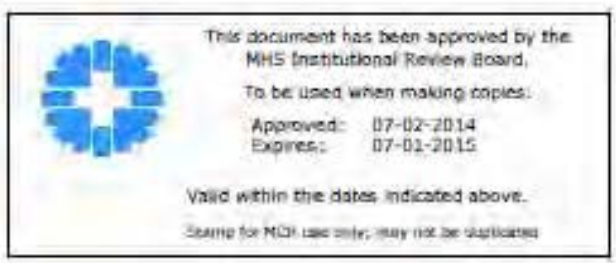


Page 7 of 8

PI: L. Tirabassi-Mathis RN PhD(c) MN CPNF

Project No. $361-14$

\section{Certificate of Person Obtaining Consent}

I have provided an explanation of the above research study and encouraged the subject to ask questions and request additional information regarding the study, its risks and complications and possible alternatives. A copy of this consent form has been given to the subject.

Signature of Person Obtaining Consent

\author{
Printed Name of Person \\ Obtaining Consent
}

Date

NOT VALID WITHOUT IRB STAMP OF APPROVAL 
Page 8 of 8

PI: L. Tirabassi-Mathis RN PhD(c) MN CPNF

Project No. 361-14

\section{RIGHTS OF HUMAN SUBJECTS IN MEDICAL RESEARCH}

Any person who is requested to consent to participate as a subject involving a medical experiment or who is requested to consent on behalf of another has the right to:

1. Be informed of the nature and purpose of the experiment.

2. Be given an explanation of the procedures to be followed in the medical experiment, and any drug or device to be utilized.

3. Be given a description of any attendant discomforts and risks reasonably to be expected from the experiment.

4. Be given an explanation of any benefits to the subjects reasonably to be expected from the experiment.

5. Be given a disclosure of any appropriate alternative procedures, drugs or devices that might be advantages to the subject, and their relative risks and benefits.

6. Be informed of the avenues of medical treatment, if any, available to the subject after the experiment if complications should arise.

7. Be given an opportunity to ask any questions concerning the experiment of the procedure involved.

8. Be instructed that consent to participate in the medical experiment may be withdrawn at any time and the subject may discontinue participation in the medical experiment without prejudice.

9. Be given a copy of any signed and dated written consent form used in relation to the experiment.

10. Be given the opportunity to decide to consent or not to consent to a medical experiment without the intervention of any element of force, fraud, deceit, duress, coercion or undue influences on the subject's decision. 
Appendix G

Authorization for the Use of Disclosure of Protected Health Information

MemorialCare Health System Institutional Review Board

Project Title: Hispanic Mothers' Stories: Narratives of Mothers

Experiences Parenting a Child with Cystic Fibrosis (CF), Health Beliefs, and Health Care Experiences

Project No: $\quad 361-14$

Sponsor: Memorial Medical Center Foundation

\section{Authorization for the Use and Disclosure of Protected Health Information}

How Will Your Confidentiality Be Protected?

You have rights regarding the privacy of your medical information collected prior to and in the course of this research. This medical information, called "protected health information" (PHI), includes demographic information, the results of physical exams, blood tests, $\mathrm{x}$-rays and other diagnostic and medical procedures, as well as your medical history. You have the right to limit the use and sharing of your PHI, and you have the right to see your medical records and know who else is seeing them.

By signing this authorization form, you are allowing the research team to have access to your $\mathrm{PHI}$ for the purposes of conducting this study. The research team includes the investigators listed on the consent form and other personnel involved in this specific study at MemorialCare Health System.

Your PHI will be used only for the purpose(s) described in the section of the informed consent entitled, "Purpose of the Study" and "Procedures".

Your PHI will be shared, as necessary, with the MemorialCare Health System (MHS) Institutional Review Board (IRB) and with any person or agency required by law. This group of people are obligated to protect your PHI.

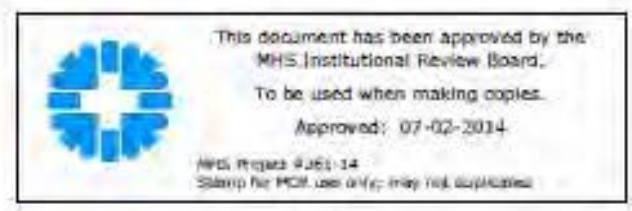


You are authorizing us to use and disclose your PHI for a period of five years after study closure.

You may revoke this authorization to use and share your PHI at any time by contacting the principal investigator in writing. If you revoke this authorization, you will no longer participate in this research program. If you revoke this authorization, use or sharing of future $\mathrm{PHI}$ will be stopped. The $\mathrm{PHI}$ that has already been collected may still be used.

The results of clinical tests and therapy performed as part of this research may be included in your medical record. The information from this study may be published in scientific journals or presented at scientific meetings but your identity will be kept confidential and no information that may be personally identifiable, such as your name, picture, social security number or medical record number, will be published.

You will be given a copy of this authorization for your records.

$\overline{\text { Printed Name of Subject }} \quad \begin{aligned} & \text { Subject Signature } \\ & \text { Date and Time }\end{aligned}$

Printed Name of Authorized Representative

Relationship to Subject

Signature of Authorized Representative

Date and Time

Printed Name of Witness

Witness Signature

Date and Time

(Applicable if document is being signed by an authorized representative)

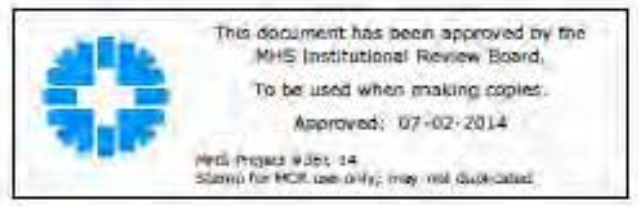

Form Rev: 03-29-2012 


\section{Appendix $\mathrm{H}$}

\section{Research Subject Reimbursement Form}

\section{MemorialCare:}

HEALTH 5 Y STEM

Exallente in Hoaltb Care
Lang Becch Memoria

Miler Children's Hospital Long Beach

Orange Coast Memcrial

Soddleback Memorial

Appendix $\mathrm{H}$

Research Subject Reimbursement Form

Subject Name: Subject ID \#

Address:

Project : : Cost Center:

Amount Due:

By signing below, I hereby acknowledge that I have participated in the above project and am entitled to receive the amount indicated.

My n/a_(relationship to subject) is aware that I am participating in a research study and I hereby give n/a (name of person) my

permission to pick up funds due on by behalf, (if needed, otherwise indicate $n / a$ )

Subject Signature and date:

Date:

Witness (PI, Coordinator or RN Signature / Title):

Date:

for castrier's omice use only

Please complete when payment is being paid to someone other than the study participant

By signature below, I have checked the identification of the individual named above that has been authorized to pick up the jembursement on behalf of the study participant.

Cashier's Signature:

Name: 


\section{Appendix I}

\section{Request for Taxpayer Identification and Certification W-9}

Identlflcatlon Number and Certlficatlon
Id

PartI Taxpayer Identification Number (TIN)

Enter your TIN in the appropriate box. The TIN provided must match the name given on the "Narne" ine to avoid backup withholding. For individuals, this is your social security number (GSN). However, for a resident alien, sole proprietor. or disregarded entity, see the Part I instructions on page 3 . For other entities, it is your employes identification number (EIN). If you do not have a number, see How to get a TIN on page 3.

Note. If the acoount is in more than one name, see the chert on pege 4 for guidelines on whcre number to enter.

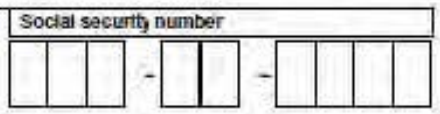

\section{Part II Certification}

\section{Under penalties of perjury, I centify that:}

1. The number shown on this form is my comect taxpayer identification number (or l am wating for a number to be issuec to me), and

2. I am not subject to bachup withholding because: (a) I am exempt from backup withholding, or (b) I have not been notified by the internal Revenue Service (IRS) that 1 am subject to backup withholding as a result of a failure to report all interest of dividends, or $|c|$ the IRS has notified me that 1 an no longer subject to backup witholding, and

3. I am a U.S. citizen or otier U.S. person (defined below), and

4. The FATCA code(s) entered on this fom (if any) indicating that 1 am exempt from FATCA reporting is correct.

Certification instructions. You must cross out item 2 above if you have been notified by the IRS that you are currently subject to backup withholding because you have failed to report all interest and dividends on your tax retum. For real estate transactions, item 2 does not apply. For mongage interest paid, acquisition or abandonment of secured property, cancellation of debt, ocotributions to an individual retirement amangement ([RA). and generally, payments other than interest and dividends, you are not required to sign the certification, but you must provide your comect TiN. See the instructions on page 3

\begin{tabular}{l|l}
\hline Sign & Signature of \\
\hline
\end{tabular}

Here $\begin{aligned} & \text { signature of } \\ & \text { u.S. person * }\end{aligned}$

Date

\section{General Instructions}

Sectibn reterences are to the interna Reverue Code uniess otterwise notad. ruture devolopments. The ine has aroatiod a page on ing. gov for information

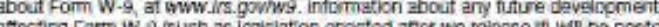
aftecting Formin-a in trat page

\section{Purpose of Form}

A person who is required to file an intormation retum weh the iRS must cotain your corect taxpayer identifoaton number (TIN) to regort. for examiple, income paid to you, payments made to you in settiament of payment card and third party network transacions, real estate transections, mortgage hterest you pald, acquisition or abendormest of secured property, cancellathan of debt, or contributions you made to an IRA.

Use Fom W-9 ony $\approx$ you are a U.S. person fincluding a resident alery, th provide yout comect tiv to the person requesting it the requester) and, when applicaive, to:

1. Certify that the TW you are giving is comect for you are wath for a number

2 Certity that you are not sibject to backup witholaing, or

3. Clam exemption trom backup with haliang if you are a U.S. exentot payee. if applicabie, you gre also certhyng unat as a U.S. person your allocable share of any partinarsinip income trom a u.S. trade $\mathrm{x}$ business ss not sutject to the
Employer identrication number 
in the cases thelow, the following person mist giva Form W-9 to the partiersitp for purposes of establinhing its U.S. status and awodirg withnoiding on its allocable share of nat income trom the partiestip condicting a trade or besines in tre uritiad statis:

- in the case of a disregarded entry witr a U.S, owner, Tre u.S, owner of the posregarded entity and not the entity.

- in the case of a grantor trust with a U.S. grantor or other U.S. uwrer, generally, the U.S, gantor of oner U, S, owner of the grantor trust and not tre trist, and - in the case of a U.S. tust (other tran a grantor trust, the U.S. trust (other than a grantor thsst) and not the beneticlaries of the thost.

Foneign person. If you are a forelgy person or the U.S. brancti of a foralgn bank. that has flected to be tratted as a U S. person, do nct Use Form W-9. Instaed, Use the approprlate Form $W$-a or Form a233 /see Pidilication 515 , With hoilung of Tax on Norrasident Allens and Foragn Entiesj.

Nonresident alien who becomes a resident allen. Generaly, cniy a nonresident allen indivdual may use tre terms of a tax iresty to redioge of Blminate U.S. tax on CBrain types of income. However, most tax treaties contain a provision show a "saking cause. Exceptichs specifiad hithe sabing cleuse may permir an has othewise tacome a US. resident aler for tax purposes.

If you are a U.S. residerit aten who is relyeng on an exseption coritained in the savirg clause of a tax treary to clalm an exempton from U.S. tax on certain types of Incorne, you must altach a statament io form W-9 that spectises the tollowing IIV sem:

1. The treaty country. Ganerally, this must be the same treaty under which you cilaimed evamption trom tax as a norresident afler

2 The trazty article addressing the income.

3. The anjcle rumber [or iocation) in the tax treaty that conialns the saving

4. The type and amount of income trat qualies for the evemption trom tax. 5. Sufficlent facts to justity the exemption tron tax under tie terns of the treaty

Example. Artcle 20 of the U.S, -China income tax treaty ancws an exempton. from tax for scholershlp inocme recelved by a Coinese student temporartly preeart

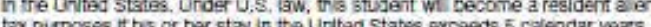
Howevor, paragran 2 of the frat Protocol to the US,-Cring treaty idated Aprl 30 ,

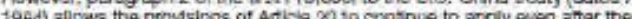
chinese studert decomes a residemt a en of the united States. A chinese studer Chinese studert deccomes a residemt a en of the united states. A Chinese studert Who qual fies for thils excapticn (under paragraph 2 of the first protocol, and is raying on this exception to cialm an exarnpton from tax on his or her scholar or fellowstip income would artach to Form W-A a statien

if you are a nonresident alen or a toreigi entry, glve the requester the apcropelate ccmpleted Form W-a or Form a23?

What is backup withholding? Pgrsons making cortain paymants to you must Inder certain condituons withodd and pay to the IAS a percentage of such payments. This is called Decklip Wrrnolding. Payments that may be subect to beckup witreioiding include interest, tax-exempt interest, dovidencs, broker and barter exchange transactions, rents, royalties, nonemployee pay, payments mad in setternent of payment card and tuiru party network transactions, and certain payments from fishing boat operators, Fiel estate transactions are not sibject to backup witholding

You wal not be surpect ti backup witholding on peyments you recalve if wou gve the requester your conses: TN., make the procee certifications, and report al

your tarable interest and dividends an your tax retum

\section{Payments you receive will be subject to backup}

\section{withholding if:}

1. You do not turnish your $\mathrm{TN}$ to the requester.

a You do not certry your TN when required / see the Part in instructons on page 3 for detalis),

3. The IAS tells the requaster that you tumished an incorrect TN,

4. The IRS tells you that you are sucject to backup withoiding Decause you did not report a your interest and divdends on your tax retim ffor reportable interest not report a your inter:

5. You do not certiry to the requester that you are not subject to beckup withoiding under 4 atxove for reportable irterest and dividerd ascounts opened ater 1963 orly

Centan payees and payments are exempt trom backup witholding. See Exempt payef croe on page 3 and the separate insfuctons tor the Requester of Fom W-9 for more intormation.

Wso see Special ruies for partherships on page 1.

What is FATCA reporting? The Foreign Aoscunt Tax Compilance Act (FATCA. requiras a partisipating fors gn thancial instifition to report al United States

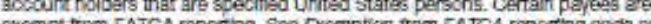
exempt irum FATCA reponting. See Evemption trom FATCA reporting code Dn

\section{Updating Your Information}

You must provide updated information to ary person to whom you claimed to be an exempt payee ef you are no longet an exempt payee and anticlpate recevirg reportable payments in the future from this person. For example, you may need provide updated information it you are a Coorporation that elects to be an $\mathrm{S}$. corpcratlon, or if you no longer are tax exempt. In additon, you must tumish a new
Form W-9 if tre name or TiN changes for the accourt, fro example, if the grantor Form W-9 if the riame

\section{Penalties}

Fallure to fumish TW. If you fal to fumish you carract $T N$ to a requaster, you are subject to a pensty of $\$ 50$ for sach such talure inlabs your falure is due to reascruble caise and not to wint negect

Civil penalty for faise information with respect to witthoiding. If you make a false stalemerit with no reasonable basis that resits in no deckup withoiding. you are subject to a $\$ 500$ penalty.

Criminai penatty for falsitying information. Wishufy falshyng certications of impriscriment

Misuse of TINs. If the requester dischoses or uses TiNs in vialatinn of federal taw the requester may be sucject to civi and cirnirisi peralfes.

\section{Specific Instructions}

\section{Name}

If you are an individual, you must ganeraly enter the name shown on your income ace refum, However, ir you nave clanged your last name, kr inct onse, due to enter your frat neme, the lest name snown on your socis securtity card, and your new last name.

If the acoourt is in joint rames, tht frst, and ther crude, the name of the person or enitly whose number you entered in Parti of tre form.

Sole propriator. Enter your indvidus narne as shown on your income tax rehim ¿DaAy name on the "Business nama/disnegarded erstly name" the.

Partnership, C corporation, or $\$$ Corporation. Enter the enttly's name on the "Name" ine and any business, trade, or "doing busiress as (DEA) name' on the

Disregarded entity. For U.S, federal tax purposes, an eritity that is disregerded as an entily separate from ts owner is treated as a "disregarded entity." See

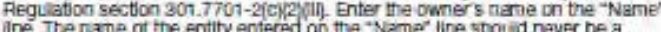
dlsregarded entty. The neme on the "Name' ine mist be the name showit on the income tax returi on which the income should be reported. For example, it a foreign $L \mathrm{C}$. that is ireated as a disregarded ertity for U.S, federal tax purposes nas a single owner that is a U.S. person, the U.S. owner's rame is required to be provided on the 'Name' ine. it the direct owree of the entty is aiso a disregarded providy, enter me first owner that is not disregarded for federal tax pupposes. Ent's

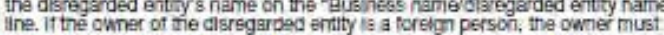
complebe an appropinate Form W- Insteat of a Fom W-B. This is the case even it the foreign persen has a U.S. TIN. Note. Check the appropniate box tor tre U.S. federsil tax classintication of the Partrersilp, c Corporation, S Corporation, Thustlegtatal.

Umited Uability Company (LC). If me parson identitsad on the "Name" ine is ar ILC, check the Umited libility compeny box ariy and enter the appropriate code for the U.S. fenera tax ciassification in the space provided. If you are an LLC that is treated as a parthesisip tor U.S. federa tax purposes, enter 'P" for pertnershlp. if you are ar Luc orat has fled a Form 3622 or a Form 2553 to be taxed as a corponation, aniter " $C$ " for $C$ Dorporatlon or " $S$ " for $\$$ cosporation, as appropriate. if you are an LLC hal is dssiggarded as an entity separate from its tak, do not check tre $4 C$ Dox uniess the owner of the LLC lrequired to be

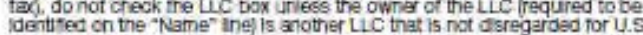
federal tax purposes. If the LLC is disreganded as an estity seperate frum is

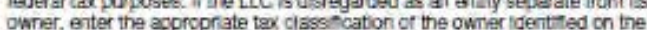
"Narne' Inie

Other entitles. Erter your business nama as stown on required U. S. Tederal tax. documants on the 'Name' lire. This hame should match the name shown on the

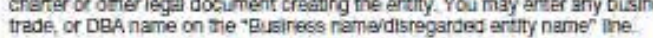

\section{Exemptions}

If you are exempt trom backup withioliting and/or FATCA reporting, erter in the Exemption from FATCA reporting code on pege 3 . 
Exempt payee code. Generally, indwiduals (incouding scle propitebors) are not exempt from dackup witholding. Corporattons are exempt fram backup witholding for Dartin payments, such as interest and divdends. Cupporations are card of third party network transaction 3 .

Note, if you are evernot trom backup wittholalng you should stil complete tris form to avold possitbe erroneous backup withinoiding.

The following codes idertity payees tiat are exempt fron beckup wthhoidng: 1-An organization exempt from tax under secton 501/al, any iPA, or a custodial account under section $403(\mathrm{~b} /(7)$ if the arsourt sattshles the requitrements of section 401010

2- The united states or any of its agencles or instrumentalties

3-A state, the District of Columbla, a possesstion of the United States, ar ary d their politica subcuisicns or instrumentanties

4-A foreign government or any of tis poubcal sibdivilons, agancles, or nstrumertailites

5-A corporation

E-A dealer in securitas or conmodioas required to regleter in the United States, me District of Columbia, or a possessicn of ge unthed States

7-A funures commisston merchart registered with the Commodty Rutures Theiling Comerilssion

B-A real estate invastment truet

9-Art ently registered at al times during the tax year under the inwestment Corpany Act of 1049

to-A common irust fund operated by a bark under saction 584(a)

11-A fnancial instifution

12-A middleman known in the investrnent community as a nominee or custodian

13-A trust axenipt trom tax under secton EBa or described in section 4947 The following chart shows types of payments trat may be exempt trum cackup withouding. The crert apcles to bie exempt clyees dsted sbove, 1 through 13.

\begin{tabular}{|c|c|}
\hline IF the payment is for ... & THEN the payment is exempt for .... \\
\hline interest and alidend paymarts & $\begin{array}{l}\text { All exempt payees except } \\
\text { for } 7\end{array}$ \\
\hline Broker trensactinns & 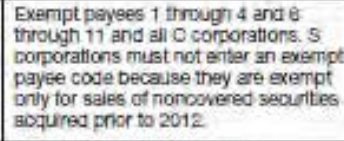 \\
\hline $\begin{array}{l}\text { Earts exchange transections and } \\
\text { patronege dlvidends }\end{array}$ & Exempt peyees 1 through 4 \\
\hline 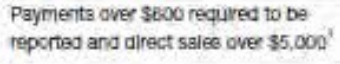 & $\begin{array}{l}\text { Generally, exempl payees } \\
\text { ittrough } 5^{2}\end{array}$ \\
\hline $\begin{array}{l}\text { Payments made in settiement of } \\
\text { payment card or third perty natwork } \\
\text { transactions }\end{array}$ & Exempt payeet 1 trrough 4 \\
\hline
\end{tabular}

'See Foim 109e-MisC, Macelarecus incorte ard ifs instruotions.

However, the following payments made to a corporation and repartatle on form tDeg-MisC are not exempt trom bachup withoiding medical and heelth pare paymerts, attomeys' fees, gross proceeds peld to ans attimey, and payments for senlces pald by a federal exacutive agancy.

Exemption from FATCA reporting code. The following codes identity payees that are exempt from reporting under FATCA. These codes apply to persons sutomitting this form for accounts maintained outside of the United States by cenain fureign finencial institutions. Therefore, if you are orry submiting this form for an acoourt you hoid in the United States, you may latve this fleld biark. Consult with the person requesting this form if you are uncertain in the nrishces insttution is sicject to these requiemerts.

A-An oryanizator exempt from tax inder section 501(a) or ary indivilua retirement pian as defired in aection 7701 (a) 37 )

B-The united states or any of its acencias or instrumental tes

C-A state, the Distnct of Columbia a possession of the Lintied states, or ary of their poiltical subdkisions or instrumentailties

D-A corporation the stock of wrich is reguarty raded on one or more

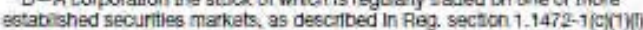

E-A corporation that is a member of the same expended artalied graip as a corporation described in Reg. serson $t .1472-1$ (15s(1)

F-A dealer in securtites, ocmmodites, or desvative financial inetrimerts (ncuosing notional principal contracts, tutures, towerds, and optings) that is reglstared as such under the lisws of the Unifed States or any state
G-A ree estate invesmart must

H-A regulated hvestment company es defhed in section 851 or an enitiy registared at al trmes curmg the ax year inder the invegimars company Act of 1940

1-A osmmon trust tund as defined in saction 584(a)

$J-A$ barik as detined in section 681

$\kappa-A$ brower

L-A trust exempt from tax under section 284 or described in 3ection 4947 (a)(1)

M-A tax exempt trust under a section ACB/D) plan or sectibn 457 (g), plar

\section{Part I. Taxpayer Identification Number (TIN)}

Enter your $\mathrm{TN}$ in the approprlate box. If you are a resident alen and you do not have and are not elighle to get an SSN, your TN is your IAS indwdua tacpayer denttlcation number (0TIN). Enter if in tre social seounty number box if you do not.

it you are a sole propriebrer and you have an EN, you may enter elther your 35 . or ElN. However, the IRS prefers that you use your SSN.

If you are a single-menber LLC that is darregarded as an enthy separate from It: owner isee Limted Llability Compary,$L C$ C) on page 24, enter the owner s SSN (O EN. it the owner has onej, Do not enter the dlsregardect erctity's classifled as a curporation or partineranip, erter the entity's EN.

Note. Sge the chart on page 4 fivr further ciatication of name and $7 \mathrm{~N}$ combirations,

How to get a TIN. if you do not nave a TIN, epply for ore immedlately. To apply for sn SSN, get Form SS-5, Applcation tor a Soclal Securtty Card, from you loca Socka Secunty Aaminstation once or get tis torm onine at whw. Ssa,gov. You may also get ins 10mm by caing 1-600-712-1213. Use form W-7, Aqpication 10 . As numidua Taupayar deant caton Nuncer, to apply for anitin, or Form ss-4, top

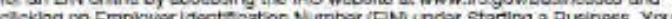
can oet Forms $W-7$ and SS-4 from the IRS by visting IRS. gov or by caling 1 -poo TAX-FORM $(1-800-829-3878)$

It you are asked to oumplete Form W-9 but do not have a TIN, apply for a TIN and writte -Appliad For in the spece for the TIN, sign and date the form, ard gvie to the requester. For interest and dividend peyments, and certain peyments made Whr regpect to resdlly tradable instruments, ganeraby you wil nave 60 days to get a TNN and glve st to tre requester betore you are sucyect to backup withrolding or Peymarts. The so-day rise does nof apply to ctie types of peyments. Vou wa te the requestes.

Note. Ertering "Apelled For means that you have already appled tor a TW or itsal you intend to sppy for one soon.

Caution: A disvegarded U.S. entily that has a forelgn owner must use the appropnate form W-8

\section{Part II. Certification}

To establsh to the witholding agent trat you are a u.S. persch, or resident ellen. sign Forn W-9. You may be requested to sign by the wtinolding agent even if For a jont account, only the person whose TIN is shawri in Parli should sigi
wher required. in the case of a disregarded entity, the person identifled on the 'Name' Ine must sign. Exempt payees, see Evempt payee cooe earler. Signature requirements. Compiebe the certiacation as indicated in items 1
trrough 5 below.

1. Interest, dlvidend, and barter exchange accounts opened before 1964 and broker accounts considered active during 1963. You must give your corect TIN. bit you do not have to sign the centincation.

2. Interest, alvidend, broker, and barter exchange accounts opened anter 1983 and broker accounts considered inactlve during 1963. You must sign the

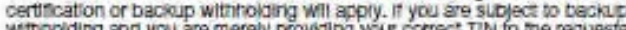

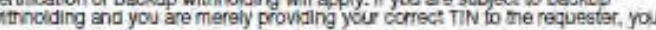
must cross out liem 2 in the certification beture signing the form.

3. Peal estate transactions. You mist sign the centificalon. You may cross ou: tern 2 of the certil cation.

4. Other payments. You must glve you correct TIN, but you do not heve ta sgn the cartification unless you nave been nothed that you have previously givan an incorect TiN. "Other payments" hiclude payments made in the course on the requesters trade or bus nest requester's trade or bus ness tor pertits, royalties, goods fother than bils for corporationsi, peyments to a ronemployent for sevicos, payments made in coporaticnsi, payments to a norem ployes icr sevices, payments made in certain flshing boat crew mernbers and fishemen, and oross procioeds paid to attorneys (including payments to corporaticns).

5. Mortgage interest pald by you, acquisition or abendonment of securec property, cancelation of dett, qualined tultion program payments (under Coyerdell ESA Archer MSA or HSA contributions or distributions, and pension distributions. You muit glve your soveret TiN. but vas do not rave to sign the certification. 
What Name and Number To Give the Requester

\begin{tabular}{|c|c|}
\hline For this type of account: & Glve neme and SSN of: \\
\hline $\begin{array}{l}\text { 1. ndwedual } \\
\text { 2. Two or more individuats joint } \\
\text { account? }\end{array}$ & $\begin{array}{l}\text { The indwdual } \\
\text { The actual owner of the accourt or, } \\
\text { if combined funds, the first } \\
\text { individual on the 30count' }\end{array}$ \\
\hline $\begin{array}{l}\text { 3. Custodian account of a minor } \\
\text { Eiviform Git to Minors Act) }\end{array}$ & The minor' \\
\hline $\begin{array}{l}\text { 4. a. The isual revocable semings. } \\
\text { trust (grartor is also trusteg) }\end{array}$ & The gantor-tusitse' \\
\hline $\begin{array}{l}\text { 0. So-caled truat accoint that is } \\
\text { not a legal or vald trust inder } \\
\text { state law }\end{array}$ & The actuas owner' \\
\hline $\begin{array}{l}\text { 5. Sole proprietorsh p or disregarded } \\
\text { entity owned by an individual }\end{array}$ & The owner" \\
\hline 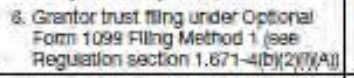 & The grantor \\
\hline For this type of account: & GWe name and EiN of: \\
\hline $\begin{array}{l}\text { 7. Ctsregarded erity not awned by an } \\
\text { inoviduat }\end{array}$ & The dwer \\
\hline a. A valid trust, estate, or persion trust & Legal entilty" \\
\hline $\begin{array}{l}\text { 9. Comporation or LLC slecting } \\
\text { corporate status on Form \&aee } \alpha \\
\text { Form } 2553\end{array}$ & The corporation \\
\hline $\begin{array}{l}\text { 70. Association, ciuh, reiliglous, } \\
\text { chariable, educational, or other } \\
\text { tax-exempt orgerzation }\end{array}$ & The orgarcation \\
\hline $\begin{array}{l}\text { 11. Partnersnip or muit-mancer } \mathrm{LC} C \\
\text { 12. A. iroker or registered nominee }\end{array}$ & $\begin{array}{l}\text { The partnership } \\
\text { The broker or nomines }\end{array}$ \\
\hline 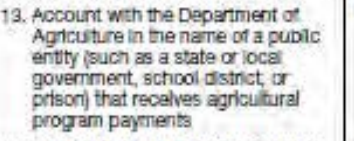 & The public entity \\
\hline 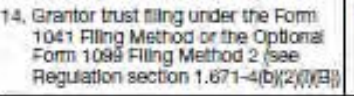 & The trust \\
\hline
\end{tabular}

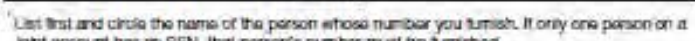

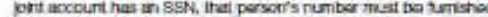

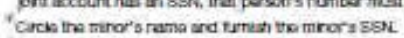

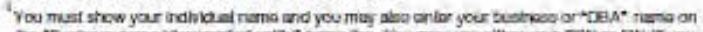

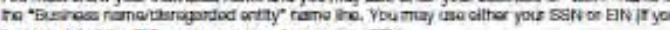

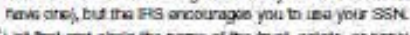

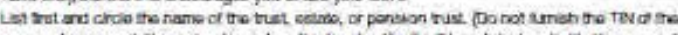

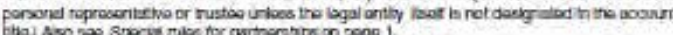

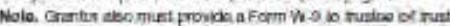

Note. If no name is circed when more than che name is ilsted, tre numicer will be considered to the that of the first neme usted.

\section{Secure Your Tax Records from Identity Theft}

identry thet occurs when someone uses your personal informatbon such as your rame soclal security number (SSM, or other idertilyng information, without your 作

To reduce your $n$ isk

- Protect your SSN.

- Ensure your employer is protecting your SSN, and

- Be caretul when choosing a tax preparer

If your tax reconde are amected by identiny then and you recave a notice trom the IRS, respond right away to the name and phore number privted on the IRS notice or letts.

If your tax records are not currently amected by idensty trent out you thati you ary at risk dive to a lost or stcien purse or walet, quastonable credit card activry

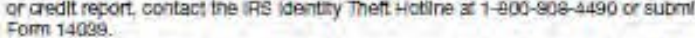

For more intormation, see Piblication 4535, identity Then Prevention and Vicum Assiatance.

Vctims of identity retr who are eypenercing economic ham or a system. proclem, or are seeking heip in resciving tax problents that have not been reeclved through noma channats, may de ellgbibe for Taxpayer Addvocale Sarvice (TAS) assistance You can feach TAS by caling the TAS toll-tee case intake line at 1-677-7T7-4778 or TTY/TDD 1-800-829-4059.

Protect yourselt from suspicious emalls or phishing schemes. Pfilsting is the cregtun and use of emall and websites designed to mimic legitimgte business emials and wercites. The most cormmon act is sending an emill to a user falseif claiming to be an established legtimate ertervise in an attempt to scam the use into sivitendering privata intormation that wis be ised for idenifly thent.

The IPS does not initiate contacts with taxpayars via enals. Also, the IAS does not request personal detaled inforr stion trough emas of ask taxpeyers for the berk or other fingncial socourts.

If you recaive an insiliched enall cleming to be from the IRS, forward this measage to phishingaiss.gov. You may elso report misuse of the iRS name, loge,

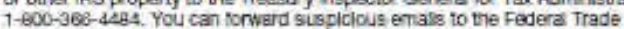

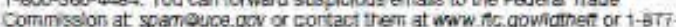
DTHEFT (1-477-438-4338).

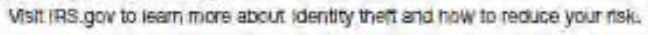

\section{Privacy Act Notice}

Section 6109 of the internal Pevenue Code requires you to provide your correct TIN to persons (ninciualing federal agencles) who are required to nle intermation retums with

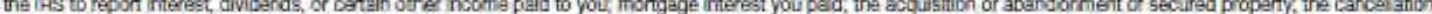
of debt, or contributions you made to an AA, Acher NSA, or HSA. The person collecting trils form uses the information on tre form to file intormation retums wis the IPS,

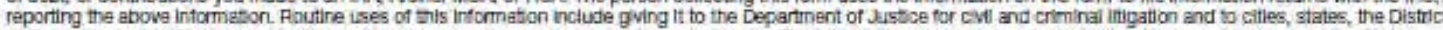
of Columble, and U.S. ocmmonweshns and possessicns for USe in aduristering thelr laws. The intomation also may be disctosed to other oounties under a treaty, to

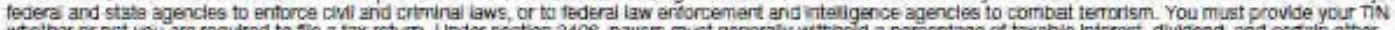
payments to a payee who does not give a TIN to the payer, Cenain penatles may also apply tor providang ferse or frauduent information. 


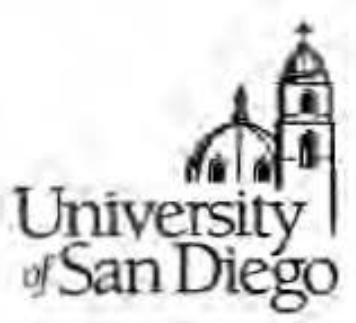

\author{
Appendix J \\ IRB Project Action Summary
}

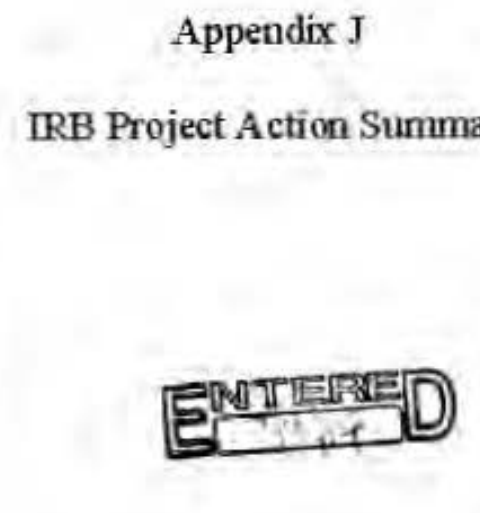

\section{Institutional Review Board Project Action Summary}

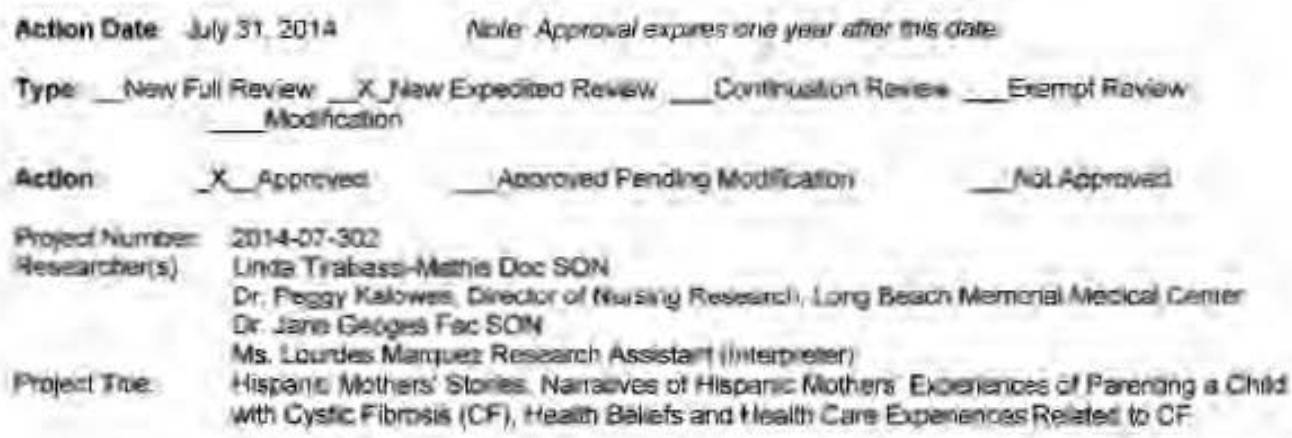

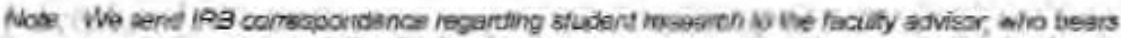

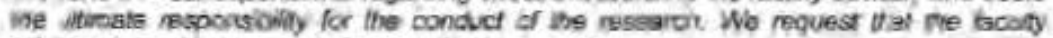

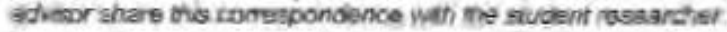

Modkications Required or Reasons for Non-Appropli

None

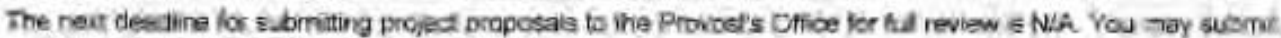
a proeect propose to Expedited review ar any äre

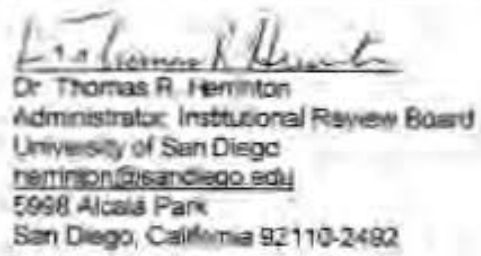

Oefoe of the Exicutive Vice Presisient and Provost 
Appendix $\mathrm{K}$

License Terms and Conditions

\section{JOHN WILEY AND SONS LICENSE TERMS AND CONDITIONS}

May 05, 2015

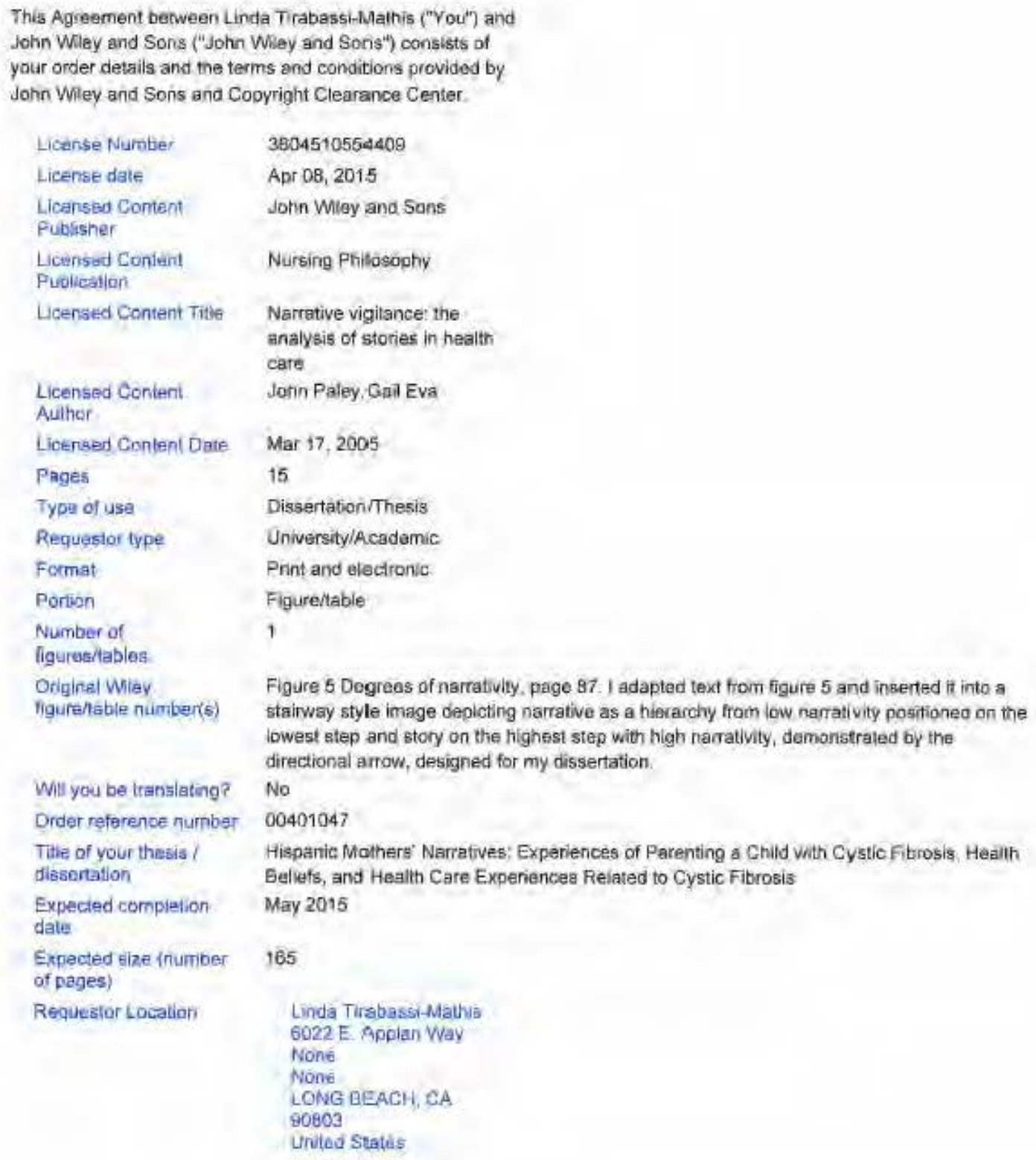

Licanse Nurnber

License dale

Licansed Content

Publisher

Licensod Conient

Puoliesilan

Licensed Content Titie

Licensed Conient

Aulher

Licerisen Conlent Date

Pages

Type of use

Requestor type

Format

Poritian

Number of

liguresitables.

Original Wiley

ngurehable number(s)

Will you be translating?

Drder reference number

Title of your thesis ?

diesertation

Expecied completion

dale

Expected size inumber of pages)

Requeztor Location

\section{9}

Apr $08,201.5$

Jotin Wloy and Sons

Nursing Philosophy

Narrative vigitance: the analysis of stories in health

care

Jorin Paley, Gan Eva

Mar 17, 2005

15

DissertationiThesis

University/Academic

Print and electronic.

Figuredtable

1

Figure 5 Degrees of narrativity, page 87 . I adapted text fiom figure 5 and inserted it into a stairway style image daplcting narrative as a hierarchy from low narrativity positionec on the lowest step and story on the highest step with high nacativity, demonistraled by the directional anow, designed for my dissertation.

No

00401047

Hispanic Mathers' Narratives: Experiences of Parenting a Child Vith Cystic fitrosis Heallh Geliefs, and Health Care Experiences Related to Cystic Fibrosis

May 2015

165

$$
\begin{aligned}
& \text { Linda Trabassi-Mathis } \\
& 6022 \text { E Applan Way } \\
& \text { None } \\
& \text { None } \\
& \text { LONG BgACH, CA } \\
& 90803 \\
& \text { Unuled Statis }
\end{aligned}
$$




\begin{tabular}{|c|c|}
\hline & \\
\hline Galing The & inveles \\
\hline Pinves Aacre & $\begin{array}{l}\text { Luda Trabassi-Manis } \\
\text { do22 E. Nppian Way } \\
\text { None } \\
\text { None } \\
\text { LONG BEACH CA S0003 } \\
\text { Lnited States } \\
\text { Alin. Unda Tirubuswi-Mathis }\end{array}$ \\
\hline Tolal & 0.00 USD \\
\hline
\end{tabular}

Terme and Complitions

\section{TERMS AND CONDITIONS}

Thie copyrigited material is owred by or axclusively icensed to John Wiloy 8 Sons, inc. or one of its group companies (each a"Wiley Company') or hándled on behalf of a spiciety with which a Wiley Company has exdivive Bubisnung nehts in ielation to a partcular woh

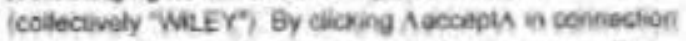
which congletirg the icenaing transacsion. you agree that the following terms and condicions apply to tivs transaction laiong wath the billing and paymant termen and openditions establistred by the Copytight Clearance Center Inc, reCCs Baing and Paymert inrms and conditions'?, at the bime that you opened your Rightslin account (these are

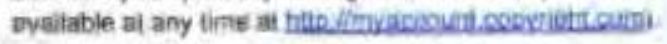

\section{Terms and Conditione}

- The materials you have requested permiesion to reprofuce or reuse (the Wiley Matariais") are protecled by sopytight

- You are hereby granted a personal, noh-exclusive non sult licensabie (on a stand-ajone basis), noniranstarsble. worldwide. Imited liciense to recroduce the Whey Materials for the purpose suectied in the koensing process. This ficense is bor a one-fine use orily and limted io any mevimum distribuivon number specilied in the license. The flist instance of repelilication or rease granted by this licence must le conpivtad wimin two years of the dase of the want of thie leence (athough copies prepared belore the end date may be distribused thereatten The Wiey Materiais shell not be used in any other mannar or for any other purpose, beyond what is granted in the ilcense. Parmission is granted subject to an appropriale acknowiedgement given to the suthor, litie of the muterial/sook/joumal and the pubsisher. Yau afail also duplicate the copyright notice that appeas in the Wiley pubication in your use of the Whey Material Permisston is alio grants i on the understonding that nowhere is the tast in a previously published source acknoviledged for ail or part of this Whay Material. Amy thind party 
content is exoreszly axcluded from this purmasian.

- YWin respect to the Whey Melerials, all rights are reserved Exoept as excoussly arantes by the tarms of the Jcenss, no pant of the Woidey Maisials may be copied, modifiert acoupled is:zeptror minor reiourating required by the new Pubication), transiated ieproduced. trankiamed or distributed. in aryy form or by any mesate and no derivative wons may be made bused on the Miey Mabriats without the prior perisiosion of the respective eopyight averier You may gol aller, remove or supposhs le any iriantier ary nopyriant. tradeinatk or oither notioes displayed by line Wiley Materiala: You may not licemse, rent, bell, bari, lease, piedge, offer sa Aecurity, trankior 2 r esten the Wilsy Materiais un a stand olone baais, sr any of the nights gianted to you fereunder to any other verson.

- The Wier Malenals and all of the intelluctual propoity riglits thereiri stabil at all times, remain vie axclusive propenty of dolin Whey \& Sons inc the Wiley Companies, or tiele tespactive ileantsats. and your intarns therain is anty that el naving poseessien of and the right to coproduco me Wiley Pxatarials pursuant to Saction 2 nersin duing the tontinuance of this. Agreertent, You agree that you cown no righti, itie af interest in of to the Wiey Malerisis or any of the inteflectual property rights therein). You shill have no rights heraunder other Whan the licentse as provided for abowe in Becilon 2 No right, license of interesto any trademen, trade aama, aarvice mark of other branding ("Marks") of WILEY or ith lioensors is gramed hereunder, and you agres that you shall not abaurt any such right. license or interest with respect thoreto.

- neitrier yiler nor its licensorg manes ANY WAARANTY OR REPRESENTATION OF ANY KNNO TO YOUOR ANY THIRD PARTY. EXPRESS IMPLIET OF STATUTORY, WTH RESPECT TO THE MATERIALS DR THE ACCURACY OF ANY INFORMATION CONTAINED IN THE MATERIALS, INCLUDING WITHOUT LIMITATION, ANY IMPLIED WARRAWTY OF MERCHANTABILITY ACCURACY, SATISFACTORV QLALITV; FITNESS FOR A FARTICULAR PLIRPOSE USABILITY, INTEGRATION OR NONINERINGEMENT AND ALL SUCH WARITANTIES ARE HEREBY EXCLUCDEO BY WILE AND ITS LICENSORS AND WAIVED BY YOL 
- WhEV slas tuve fe nght la tarmirate inir Agreenent immediabely upas Biesuli of this Agreeneri by you.

- Tou stiall indirrmity, Geiend and hoid narmiesa WLEY As Loensors and these respocive thirectors. officens, agerits and onsicvess, from Fnd aganst any actual or inrealeneo craims,

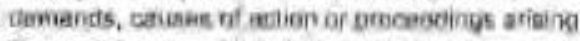
from any Breach of this Agreement by you.

- IN NO EVENT SHALL WILEY OR ITS LACENSORS BE LAABLE TO YOU OR AMY ITHER PARTV OR ANY OTHER PERSON OR ENTITY FOR ANY SPECIAL, CONSEQUENTIL. NCIOENTAL NDIRECT, EXEMMLLRY OR PUNITIVE DAMAGES, HOWEVER CAUSEO ARISINO OUT OF OA IN CONNECTION WATH THE DOWNLOADING. PRONISIONING. VHEWNG OR USE OF THE MATERIALS RECAROLESS OF THE FORM CF ACTON WHETIER FOR AREACIT OF CONTRACT, RREACH OF WARRANTY TORT, NEGLIGENCE WWFRINGEMENT OR OTHERWSE (INCLUDING WTHOUTLIMITATION, DAMACES BASED ON LOSS OF PROFITS, DATA FILES LISE. BUSINESS OPRORTUNITY OR CLAIMS OF THIRD DARTIES). AND WMETMEH OR NOT THE PARTV HAS EEEN ADVISED OF THE PDSSIALITY OF SUCH DAMAGES THIS LMMTATION SHALL APPLY NOTWTTHST ANDING ANY FAILLARE OF ESSENTIAL PURPOSE OF ANV LIMTEO REMEDY PAOVIDED HEREIN

7. Should any provison of this Agroment be had by

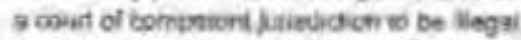

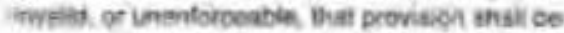
deentad smanited to acheves as reariy as possiblo

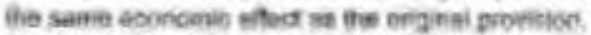
ard the iegsidy, vaiesty and entofcrabisiv of the remaining provecions of inis Aqueeriert stual not. be affected of inpmired thereby

- The failue of either nurtv to entorce any term of condition of this Agreenent ahall net cansature a waher of silter partys fight to entoros each and every ierm and corvilion of this Agreement. No brepth under this aproemem shail be deemed: walved or excused by enther party uniess such waver of boment is in wniteg samed by the parfy grenting suafi waiver or cunsent. The waiver by of censers of a party to a breach of any provesion of this Agresment anall not cperale or be constived as 9 waiver of or consenet is any ahner of mosecterst brench by wish other party. 
2. Tha Agreement may not be asaigned inolicing of overalion af low or otnerwise) by you wiltroit WIEV's prior winen consont.

- Any loe requeded for this pramingion shai ba nim relundabie atiar thiry (3e) days fiom ranepl by ite dec.

2. These terms and conditiona together with CCCAs Biating ana faymant taims and oonititions (which arp hoorpoested havain) form the entipe agreemani benween you and WLEY Danceming this lliseising transaction and (in the absence of fraud) superaedas sil pooe agraemants and representations of the parties, ofal of written. Thas Agreochant may not be amendad except in writing signed by both perties. This Agreertent slisil be binding voon and imire io the borieh of the parties? sulpkabors, lecual representatives and authorized assigus

- in the event of any contiact bewweary yous obilgations estabushed by these terms and cortititone and those established oy CCCAs biling and Payment tarms and conditions, these lesms ate conclitons shall prevaili

- WILFy expressly reserves all ighis rot sptcilically पranted in the combination of (i) the liosise detalit: pavided by you and accepled in the couno of thes licensing inansaction, (ii) betese lemis and sonditions and (ih) CCCA s Bitang and Paymen lems and conditions.

1. This Ryresment will br void if the Type of Use Format Croulation a Requestor Type was miscepresented during tiv licenseg croess.

This Agreament shall be governed by and consinued in accordanse with the laws of the Stale of Now Vork. USA, without regards to such siareas cunlict of law rules: Any legal action sua or pluceeding ariaing out of or relating to these Terme and Conoitions or the brasech thersof shall be inatlutied in a court of competent jursedictiow in New Yonk County in the state of New York in the United Stales of Amacica and each parily hereby consents and submis to fie persmat paritensation of auch court, waves any objection to vanua in such count and coimenta in service of process is tegietared or certsied meil, return recaiol requested ith the lesi known address of stich. pariy.

\section{WLEY OPEN ACCESS TERMS AND CONOITONS}


Wiley Publishes Open Access Avicies in fulty Open Access Journais and in Subacription joumsis offerng Online Open. Although most of the haly Open Access jourtads pubis/l open access articles under the terins of the Crestive Commone Atvibution (CC BY) Ucanse only, the supscription journale and a few of the Open Access sourrats offer a choica of Creative Commons Lipensas: Creative Commoms Attnbution (CC-BY) license Cfeteluse

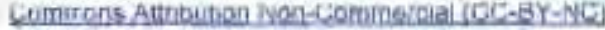
licantit and Creative Commons Attribution Non: Commeraal-ovoCerivs iCC-BY-NG-NDi Licanse. The license type is ciearly identified on the articte.

Copyrght in any research article in a joutral published as Open Access under a Creative Commons Ligense is retained by the author(s) Authous grant Wiey a licerse fo publish the articie ano identify itself as the ariginal publiaber. Authors also grant any third party the nignt to use the article fresty as long as its intognty is maintained and ta orignel athors, ditation datails and publisher sos identifed as follows: (fitie of Artucie/Author/Journal The and Volume/saue. Copyright (c) Lyear) loopyright owner as specified in the Joumall. Links to the finat article on Wiley is wehsite are encouraged where apolicable.

The Creative Commens Attribution License

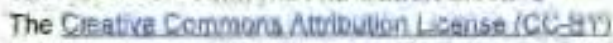
allows users to copy, distribute and transmit an article sdapt the article and make comrercial use of the siticle The CC.BY lisense permits sommercial and noncommercial re-use of m open accoss article ss iong as the author is properly attributed.

The Creative Comimons Aunbution ticense does nol affert the maral rghts of authors, inciuding without limilation the night not to have their work subjecied to darogatory treatment. it also does not sffect any other rights held by authons or thind perties in the article, including without limitetion the fights of privacy and publicity. Use of the article nust not assert or imply, whethar implicilly or explicity, any connectorn with, endorsament on sponscrstio of such use by the aulfior, publisher or any other panty associeted with the articte.

For any revise or detribotion. users munt include the copyright notice and make ciear to others that the arbele is made iviliabie under a Croalive Commons Altribulinn lloense, linking to the raievant Creative Commans web page.

To the fullest exieni parmilted by applicable law, the article is made avaliable as is and without representation or wamanties of any kind wheither express, impled. stalutory or otherwise and mcloding, without imitation. warranies of bitle, merchantabillty. Miness for a partecular purposa, non-tafringamert, absence of dufects, accuracy. or the proserice of absence of errors

Creative Commons Atrribution Non-Commerciai License

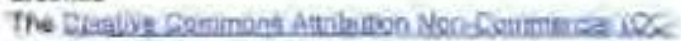
Byctich Lenatine pemile use, diairibation and reproduction 
in any medium, provided the oripinal work is properly cled and ia not Laed for commercial purnoses (see below) Creative Commons Attribution-Non-CommerciatNoDorivs License

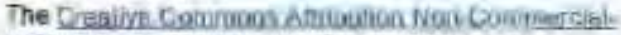
UaDeing Ligrnse (CC-BY-NC-ND) parmits Lae dismibution and reproduclion in any mediuin, provided the onginel wort is properiy ciled, is nof used for curvmenvial purpotes and no modifications or adaptations are marte (see bslow)

Use by non-eommerelal usen

For noh-commerelal and non-promotional purposes individual users may access. downioed, copy diaplay and rectiatibute to colleagues Wiley Open Acoass anticles as well at adapt. Iranslate, fext-and data-mine the content subjed to the following conditions:

- The auiters' moral righte aro not compromised. These rights lischide the right of "Daternily" istso known as 'atrituation' - the reght for the puthor to be identified as suchi and "iniegrity" the rioht lor the author nat to have the won allered in suck a Way that the author's reputation of integrey may be impugned).

- Mere conient in the article is identtiert as beimrging to a thind pariy, $A$ it the obilgation of the vaer to knsure that any reuse complies inth the sopynght policies of the owner of that content.

- If artiole content is copied, downipaded ar omervise feused for non-commercial research and aducatien purposes, a link to the appropriste bibliographic citation fauthors, journal, article title. volime, issue, page numbers. DOl and the link la the definitive pubilished version an Wiley Ontine Ulbrary) should be maintained. Copynght nolices ant disclaimen must not be deleted.

- Any lansiations, for which a prior translation agreament with Wiley has not been agreed, must pinminently display the stalement. Thie is an unoffeia: iransintion of an artede that appeared in a Wiay publication The publigher has noi endorsed inis transietion."

Use by commerclat "for profit" organisation Lse of Miey Doen Acoess articies for commercial, prornotonal, or misketing puiposes requires further explicit permission from Wiley and will be sutject io a lee Commarcial purposes include:

- Copying or downioading of ariales, se Inkung to sucti aflicies for furthes redistribution. sale br Bcansing. 
- Copying downdoadang or posting by a sle or service that incomoratas advertising with such content:

- The inclusion or incorporation of article contert in ather works or senvices (other than nornial quotations with an appropriate citation) that is then avaltabie for saie or ficensing for a fee ffor axarnple, a compiation produced for marketing purposes, inclusion in a saies pack)

- Vae af articie content (other than normal quotetions with appropriate cikation) by for-provil organikatione for premotional purposes

- Linking to articie contant in e-mais fedistrituted for promational. marketing or educationai purposes.

- Use for the purposes of monetary reward by means of sale, resaie, licence, loan transfer or other form of commercial explotitaticn Euch as makeling products

- Preif renfiots of Wiey Ópen Access arlides can be purchased from. cevoralesales (Inwikgoem

Furtne diataili can be found on Whey Onine Liarary

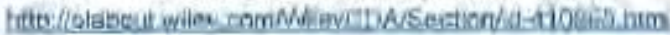

Other Tomis and Conditions:

v.9

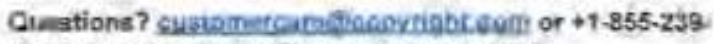
3415 (toll free in the US) of $* 1-978-645-2777$.

Gratis licenses (referencing $s 0$ in the Total feld) are trate Please retain this printabie license for your reference. No payment is required. 
